Thadeu das Neves Conti

\title{
APLICAÇÃO DO MÉTODO DA EXPANSÃO EM FUNÇÕES HIERÁRQUICAS NA SOLUÇÃO DAS EQUAÇÕES DE NAVIER- STOKES EM DUAS DIMENSÕES PARA FLUIDOS COMPRESSÍVEIS \\ EM ALTA VELOCIDADE
}

\author{
Tese apresentada à Escola Politécnica da \\ Universidade de São Paulo para obtenção do título \\ de Doutor em Engenharia.
}


Thadeu das Neves Conti

\section{APLICAÇÃO DO MÉTODO DA EXPANSÃO EM FUNÇÕES HIERÁRQUICAS NA SOLUÇÃO DAS EQUAÇÕES DE NAVIER- STOKES EM DUAS DIMENSÕES PARA FLUIDOS COMPRESSÍVEIS EM ALTA VELOCIDADE}

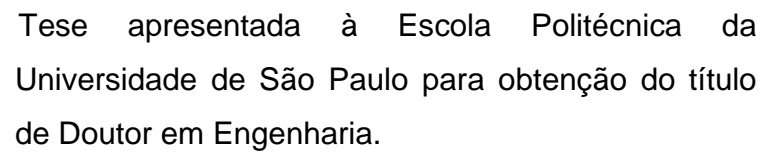

Área de concentração: Engenharia Mecânica 
Este exemplar foi revisado e alterado em relação à versão original, sob responsabilidade única do autor e com a anuência de seu orientador.

São Paulo, 8 de julho de 2006

Assinatura do autor

Assinatura do orientador

FICHA CATALOGRÁFICA

Conti, Thadeu das Neves

Aplicação do método da expansão em funções hierárquicas na solução das equações de navier-Stokes em duas dimensões para fluidos compressíveis em alta velocidade I T. das N. Conti. -- São Paulo, 2006.

$131 \mathrm{p}$.

Tese (Doutorado) - Escola Politécnica da Universidade de São Paulo. Departamento de Engenharia Mecânica.

1.Mecânica dos fluidos computacional 2.Escoamento monofásico 3.Método dos elementos finitos 4.Equações de NavierStokes 5.Ondas de choque I.Universidade de São Paulo. Escola Politécnica. Departamento de Engenharia Mecânica II.t. 
À minha esposa Eliana, e aos meus filhos Thiago e Júlia. 


\section{AGRADECIMENTOS}

Ao Prof. Dr. Eduardo Lobo Lustosa Cabral, pela competente, segura e honesta orientação demonstrada durante toda a realização deste trabalho.

À minha esposa Eliana e aos meus filhos Thiago e Júlia, pelo amor, ajuda, encorajamento e compreensão que sempre me dedicaram, principalmente durante o curso de Pós-Graduação, fundamentais para a concretização deste trabalho.

Aos meus pais Guiomar e Carlos (em memória), pelo amor e carinho que sempre me dedicaram.

À minha amiga Prof ${ }^{\mathrm{a}}$. Dr ${ }^{\mathrm{a}}$. Gaianê Sabundjian, pela amizade e pelo incentivo durante a realização deste trabalho.

Aos meus professores do curso de Pós-Graduação da Escola Politécnica da Universidade de São Paulo, por tudo que ensinaram, em suas aulas, de maneira inesquecível.

Aos professores Dr. José Roberto Simões Moreira, Dr. Júlio Romano Meneghini e Dr. Ernani Vitillo Volpe, pelas valiosas contribuições durante a realização deste trabalho.

Ao Departamento de Engenharia Mecânica da Escola Politécnica da Universidade de São Paulo, pela oportunidade e pelo apoio que sempre me deram em todos os momentos.

Ao Centro do Reator de Pesquisas e ao Centro de Engenharia Nuclear do Instituto de Pesquisas Energéticas e Nucleares - Comissão Nacional de Energia Nuclear, pela oportunidade e apoio irrestrito que sempre me dispensaram. 
Aos amigos e aos colegas da Escola Politécnica da Universidade de São Paulo e do Instituto de Pesquisas Energéticas e Nucleares que, direta ou indiretamente ajudaram na elaboração desta tese. 


\section{RESUMO}

O trabalho desenvolvido nesta tese propõe a aplicação do método da expansão em funções hierárquicas elaborado por Zienkiewics e Morgan (1983), para a solução das equações de conservação da massa (continuidade), conservação da quantidade de movimento (Navier-Stokes) e conservação da energia, para fluidos compressíveis em duas dimensões e em alta velocidade. Esse método consiste no emprego do método de elementos finitos utilizando a formulação Petrov-Galerkin, conhecido como SUPG ("Streamline Upwind Petrov-Galerkin”), desenvolvido por Brooks e Hughes (1982), aplicado em conjunto com uma expansão das variáveis em funções hierárquicas. A fim de testar e validar o método numérico proposto, assim como o programa computacional elaborado, foram simulados alguns casos conhecidos da literatura. Os casos estudados foram os seguintes: teste de Continuidade; teste de convergência e estabilidade; problema do degrau de temperatura e problema do choque oblíquo, onde o objetivo desse último caso era, basicamente, verificar a captura da onda de choque pelo método numérico desenvolvido. Através dos casos estudados e em função dos resultados obtidos nas simulações realizadas, conclui-se que o objetivo desse trabalho foi alcançado de maneira satisfatória, pois os resultados obtidos com o método desenvolvido nesse trabalho foram qualitativamente e quantitativamente bons, quando comparados com os resultados teóricos. 


\begin{abstract}
The Thesis develops a new application for the Hierarchical Function Expansion Method, proposed by Zienkiewics and Morgan (1983), for the solution of the NavierStokes equations for compressible fluids in two dimensions and in high velocity. This method is based on the finite elements method using the Petrov-Galerkin formulation, know as, SUPG (Streamline Upwind Petrov-Galerkin) developed by Brooks and Hughes (1982), and applied in conjunction with the expansion of the variables into hierarchical functions. To test and validate the numerical method proposed as well as the computational program developed some cases whose theoretical solution are known simulated. These cases are the following: continuity test; stability and convergence test; temperature step problem; and several oblique shocks. The objective of the last cases is basically to verify the capture of the shock wave by the method developed. The results obtained in the simulations of the cases performed with the proposed method were good both qualitatively and quantitatively when compared with the teorethical solutions. This allows us to conclude that the objective of this Thesis was satisfactorily reached.
\end{abstract}




\section{SUMÁRIO}

RESUMO

ABSTRACT

LISTA DE FIGURAS

NOMENCLATURA

$\begin{array}{ll}\text { 1- INTRODUÇÃO } & 18\end{array}$

$\begin{array}{ll}1.1 \text { - Objetivo do trabalho } & 20\end{array}$

2- REVISÃO BIBLIOGRÁFICA

3- EQUAÇÕES DE CONSERVAÇÃO 32

3.1 - Equação de conservação da massa (continuidade) 32

3.2 - Equação de conservação da quantidade de movimento 32

3.3 - Equação de conservação da energia 37

3.4 - Equação de estado $\quad 39$

3.5 - Equações de conservação em duas dimensões 39

4- DESENVOLVIMENTO TEÓRICO 41

4.1 - Discretização do domínio de solução 43

4.2 - Desenvolvimento matemático das equações de conservação para $\begin{array}{ll}\text { um elemento } & 45\end{array}$

4.2.1 - Equação da continuidade $\quad 49$

4.2.2 - Equação de conservação da quantidade de movimento na

direção x 
4.2.3 - Equação de conservação da quantidade de movimento na direção z

4.2.4 - Equação da velocidade mássica na direção x

4.2.5 - Equação da velocidade mássica na direção z

4.2.6 - Equação de estado

4.2.7 - Equação de conservação da energia 63

4.2.8 - Equação da temperatura

4.3 - Funções de expansão 68

4.3.1 - Funções de expansão clássica 69

4.3.2 - Funções de expansão hierárquicas em uma dimensão 73

4.3.3 - Funções de expansão hierárquicas em duas dimensões 76

4.4 - Processo de agrupamento 79

4.5 - Algoritmo de Solução 84

5- RESUltados

5.1 - Teste de Continuidade $\quad 86$

5.2 - Teste de Consistência e Estabilidade 92

$\begin{array}{ll}5.3 \text { - Problema do Degrau de Temperatura } & 101\end{array}$

$\begin{array}{ll}5.4 \text { - Problema do Choque Oblíquo } & 105\end{array}$

5.4.1 - Choque Oblíquo Reto - Solução Natural 106

5.4.2 - Choque Oblíquo Reto - Solução Forçada 109

5.4.3 - Choque Oblíquo Curvo 112 
6- CONCLUSÕES 114

$\begin{array}{lr}\text { 6.1 - Conclusão } & 115\end{array}$

6.1 - Trabalhos Futuros 116

ANEXO A - FUNÇÕES DE EXPANSÃO HIERÁRQUICAS UTILIZADAS

$\begin{array}{ll}\text { NO PMEFH2D } & 117\end{array}$

REFERÊNCIAS BIBLIOGRÁFICAS $\quad 124$ 


\section{LISTA DE FIGURAS}

Figura 3.1 - Elemento de fluido em movimento

Figura 4.1 - Esquema da malha retangular utilizada

Figura 4.2 - Elementos unidimensionais e funções de expansão, (a) linear,

(b) quadrática e (c) cúbica, segundo Zinkiewics e Morgan (1983)

Figura 4.3 - Elementos hierárquicos e funções de expansão quase ortogonais,

(a) linear, (b) quadrática e (c) cúbica

Figura 4.4 - Elemento retangular e algumas funções de expansão hierárquicas associadas

Figura 4.5 - Elemento retangular de duas dimensões e seus parâmetros associados

Figura 4.6 - Quatro elementos adjacentes e seus coeficientes de expansão associados

Figura 5.1 - Domínio computacional (0,5 m x 0,5 m) - Escoamento de ar à alta velocidade na direção $x$ da esquerda para a direita

Figura 5.2 - Domínio computacional (0,5 m x 0,5 m) - Escoamento de ar à alta velocidade na direção $z$ de baixo para cima

Figura 5.3 - Domínio computacional (0,5 m x 0,5 m) - Escoamento de ar à alta velocidade nas direções $x$ e $z$ com ângulo de inclinação igual a $45^{\circ}$

Figura 5.4 - Comportamento da pressão referente ao domínio computacional da figura 5.1 
Figura 5.5 - Comportamento da temperatura referente ao domínio computacional da figura 5.2

Figura 5.6 - Comportamento da velocidade u referente ao domínio computacional da figura 5.3

Figura 5.7 - Comportamento da velocidade $w$ referente ao domínio computacional da figura 5.3

Figura 5.8 - Domínio computacional (1,2 m x 1,2 m) do teste de convergência

e estabilidade

Figura 5.9 - Comportamento da velocidade $u$ (normal ao choque) - malha computacional com 100 células

Figura 5.10 - Comportamento da velocidade $w$ (tangencial ao choque) malha computacional com 100 células

Figura 5.11 - Comportamento da pressão - malha computacional com 100 células

Figura 5.12 - Comportamento da temperatura - malha computacional com 100 células

Figura 5.13 - Comportamento da velocidade $u$ (normal ao choque) - malha computacional com 400 células

Figura 5.14 - Comportamento da velocidade $w$ (tangencial ao choque) malha computacional com 400 células

Figura 5.15 - Comportamento da pressão - malha computacional com 400 células 
Figura 5.16 - Comportamento da temperatura - malha computacional com 400 células

Figura 5.17 - Comportamento da velocidade $u$ (normal ao choque) - malha computacional com 1600 células

Figura 5.18 - Comportamento da velocidade $w$ (tangencial ao choque) malha computacional com 1600 células

Figura 5.19 - Comportamento da pressão - malha computacional com 1600 células

Figura 5.20 - Comportamento da temperatura - malha computacional com 1600 células

Figura 5.21 - Figura 5.21 - Domínio computacional (2,0 m x 2,0 m) do problema do degrau de temperatura

Figura 5.22 - Comportamento da temperatura - malha computacional com 100 células e grau 1 na expansão das variáveis

Figura 5.23 - Comportamento da temperatura - malha computacional com 100 células e grau 2 na expansão das variáveis

Figura 5.24 - Comportamento da temperatura - malha computacional com 100 células e grau 3 na expansão das variáveis

Figura 5.25 - Comportamento da temperatura - malha computacional com 100 células e grau 4 na expansão das variáveis

Figura 5.26 - Domínio computacional (1,0 m x 1,0 m) do problema do choque oblíquo reto - solução natural 
Figura 5.27 - Comportamento da velocidade de escoamento junto à interface de separação das regiões 1 e 2

Figura 5.28 - Comportamento da onda de choque - choque oblíquo reto (solução natural), utilizando grau 1 na expansão das variáveis

Figura 5.29 - Comportamento da onda de choque - choque oblíquo reto (solução natural), utilizando grau 2 na expansão das variáveis

Figura 5.30 - Domínio Computacional (1,0 m x 1,0 m) do problema do choque oblíquo reto - solução forçada

Figura 5.31 - Comportamento da onda de choque - choque oblíquo reto (solução forçada), utilizando grau 2 na expansão das variáveis

Figura 5.32 - Domínio Computacional (1,0 m x 1,0 m) do problema do choque oblíquo curvo

Figura 5.33 - Comportamento da onda de choque - choque oblíquo curvo e deslocado do corpo, utilizando grau 2 na expansão das variáveis

Figura A.1 - Elemento retangular de duas dimensões e seus parâmetros associados 


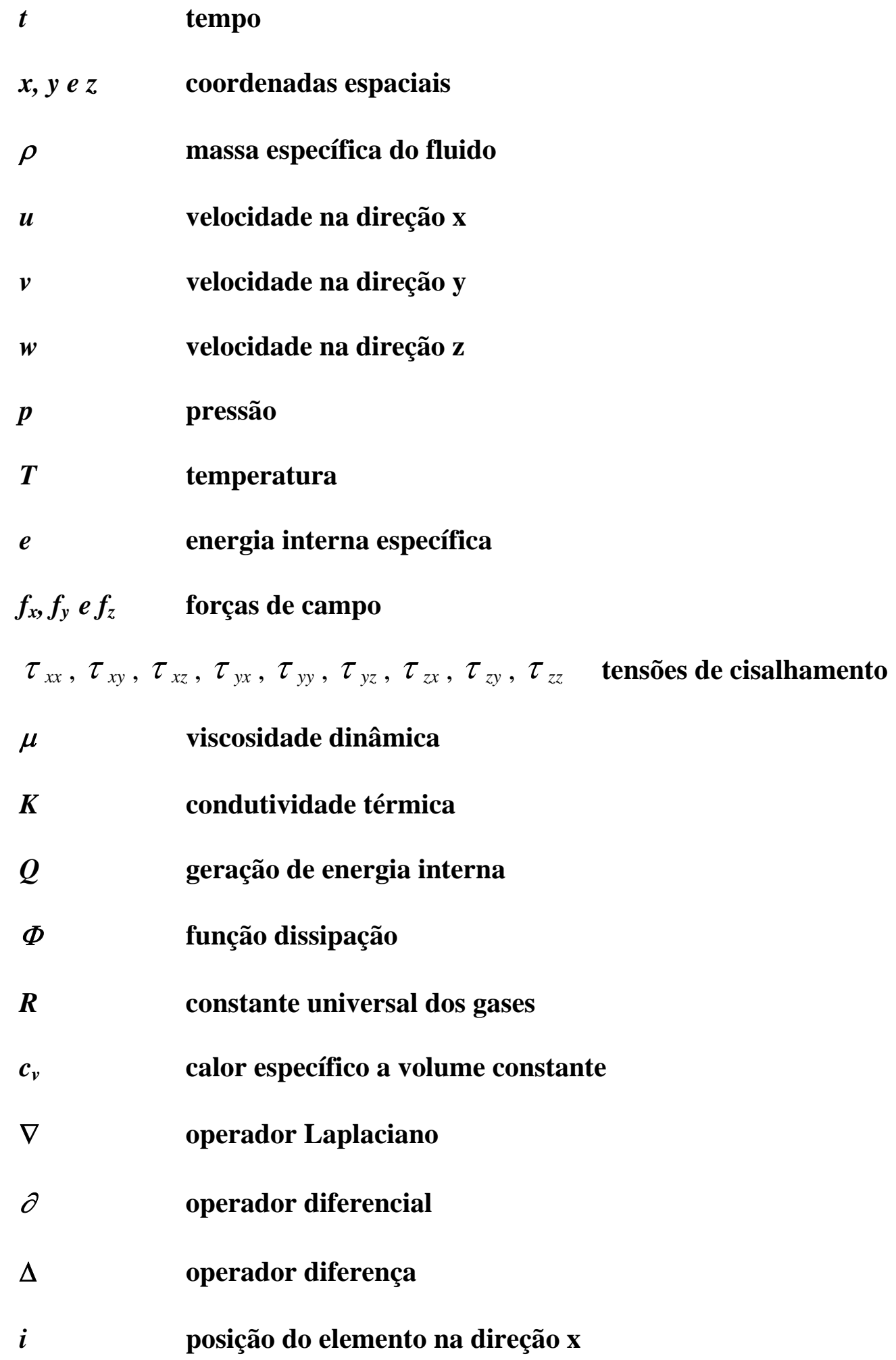


posição do elemento na direção $\mathrm{z}$

$\xi$

mudança da coordenada $x$

$\eta$

mudança da coordenada $\mathrm{z}$

V

volume

$\boldsymbol{P}_{m}$

função peso

$N_{m}$

função de expansão

$A_{m, n}^{i j}$

matriz de expansão

$b_{m, n}^{i j}$

vetor

$\alpha_{m, n}^{i j}$

integral de volume

$\beta_{m, n}^{i j}$

integral de volume

$\gamma_{m, n}^{i j}$

integral de volume

$\Omega_{m, n}^{i j}$

integral de volume

$\theta_{m, n}^{i j}$

integral de volume

$\psi_{m, n}^{i j}$

integral de volume

$C_{m, n}^{i j}$

integral de volume

$D_{m, n}^{i j} \quad$ integral de volume

CFD “Computational Fluid Dynamics”

CPU Unidade Central de Processamento

SUPG “Streamline Upwind Petrov-Galerkin”

MDF Método das Diferenças Finitas 
MVF

MEF

FCT

ALE

PFFT

UGN

E-CUSP
Método dos Volumes Finitos

Método dos Elementos Finitos

Método de Transporte de Fluxo Corrigido

Formulação - “Arbitrary Lagrangian Eulerian Equations”

algoritmo pré-corretor de Transformada de Fourier

tipo de formulação numérica

tipo de formulação numérica 


\section{1 - INTRODUÇÃO}

A solução de problemas complexos das áreas de mecânica dos fluidos e transferência de calor através do uso de técnicas numéricas, conhecida como “Computational Fluid Dynamics (CFD)”, é, hoje, uma realidade, graças ao desenvolvimento de computadores de alta velocidade de processamento e de grande capacidade de armazenamento de dados. Em função desse avanço computacional, o desenvolvimento de algoritmos para a solução de problemas, referentes às mais diversas áreas, tem-se tornado um fato e recebido muita atenção pelos analistas numéricos, Maliska (1995). Como exemplo, pode ser citado a solução de um escoamento turbulento sobre um aerofólio, que usando computadores de grande porte na década de 60 consumiria anos de processamento a um custo de milhões de dólares. A solução do mesmo problema, nos dias de hoje, utilizando computadores atuais, consumiria apenas alguns minutos de CPU a um custo de centenas de dólares.

O trabalho, aqui desenvolvido, propõe a aplicação do método da expansão em funções hierárquicas, elaborado por Zienkiewics e Morgan (1983), para a solução das equações de Navier-Stokes para fluidos compressíveis em duas dimensões em alta velocidade. Esse método consiste no emprego do método de elementos finitos utilizando a formulação Petrov-Galerkin em conjunto com uma expansão das variáveis em funções hierárquicas. A utilização de funções de expansão hierárquicas permite alterar o grau do polinômio de ajuste das variáveis, como ocorre no método de elementos finitos convencional. Além disso, o método numérico proposto tem a grande vantagem de poder adaptar o grau do polinômio até o valor necessário ou desejado, ao invés de usar malhas extremamente refinadas.

De um modo geral o método clássico dos resíduos ponderados (Galerkin) é utilizado para obter as equações e os coeficientes das funções de expansão. No entanto, observa-se que em problemas de transporte convectivo-difusivo, com convecção predominante, o método de Galerkin é falho. Assim, normalmente, é utilizada a formulação 
Petrov-Galerkin, onde as funções peso são diferentes das funções de expansão, o que difere da adotada na formulação Galerkin.

A formulação Petrov-Galerkin consiste no método conhecido como SUPG ("Streamline Upwind Petrov-Galerkin”), desenvolvido por Brooks e Hughes (1982). Nesse método as funções peso são construídas adicionando-se às funções peso da formulação de Galerkin uma perturbação que atua somente na direção do escoamento. Segundo Zienkiewicz et al. (1976), este método apresenta estabilidade e precisão nos resultados.

Baseado na revisão bibliográfica realizada, evidencia-se a originalidade do método numérico desenvolvido neste trabalho para a solução das equações de Navier-Stokes em duas dimensões, para fluidos compressíveis em alta velocidade, com captura da onda de choque. No entanto, deve-se ressaltar que o precursor do emprego deste método foi Cabral (1996), que utilizou, com sucesso, o método das funções de expansão hierárquicas para resolver a equação de difusão de nêutrons em dois grupos de energia, em duas dimensões. Posteriormente o método também foi utilizado por Sabundjian e Cabral (1999) na solução das equações de Navier-Stokes para fluidos incompressíveis em duas dimensões, com escoamento em regime laminar.

O método da expansão em funções hierárquicas foi elaborado por Zienkiewiscz e Morgan (1983), sendo que a sua grande vantagem está em poder alterar o grau da expansão sem a necessidade de reiniciar o problema. As funções de expansão são formadas por polinômios de Legendre, os quais são ajustados nos elementos retangulares, de forma a definir funções de canto, de lado e de área. A razão principal de serem utilizados, neste trabalho, os polinômios de Legendre como base das funções de expansão, ao invés de funções ortogonais do tipo seno, cosseno ou exponencial, é porque um número menor de funções é exigido quando se usa polinômios. Isso ocorre, pois um polinômio é mais complexo do que as outras funções e, comparativamente, tem uma maior capacidade de ajuste. Observa-se que, quanto menor for o número de funções utilizadas (necessárias), mais simples se torna o método numérico. 


\section{1 - Objetivo do trabalho}

O objetivo deste trabalho é desenvolver um método numérico, baseado no método dos elementos finitos, que utiliza a formulação Petrov-Galerkin ("Streamline Upwind Petrov-Galerkin” - SUPG ) para a solução das equações de Navier-Stokes em duas dimensões, para fluidos compressíveis em alta velocidade. Nesse método, como proposto por Zienkiewics e Morgan (1983), a expansão das variáveis é realizada por funções hierárquicas. 


\section{2 - REVISÃO BIBLIOGRÁFICA}

Os métodos numéricos existentes na literatura mais utilizados para o tratamento das equações de Navier-Stokes, na solução dos mais diversos tipos de problemas de engenharia, são o método das diferenças finitas (MDF), o método dos volumes finitos (MVF) e o método dos elementos finitos (MEF).

Da literatura, sabe-se que o método dos elementos finitos foi, durante muito tempo, preterido em relação ao método de diferenças finitas, quando o problema analisado era da área de mecânica dos fluidos. A razão dessa pouca utilização foi o insucesso das primeiras experiências de aplicação desse método a essa classe de problemas.

Embora o MEF fosse uma boa ferramenta para o tratamento de geometrias complexas, não possuía a habilidade necessária para o tratamento dos termos convectivos presentes nas equações de movimento. Por esse motivo, durante muito tempo, o método de diferenças finitas foi utilizado para o tratamento de problemas da mecânica dos fluidos em geometrias simples, quando se iniciou o aprimoramento do método dos volumes finitos (MVF), no qual as equações aproximadas são obtidas através do balanço das grandezas em questão (massa, quantidade de movimento, energia, etc.), nos volumes elementares ou volumes finitos. Esse método, por sua vez, era utilizado para a solução de problemas de fluidos, que envolviam geometrias mais complexas. Tanto o MDF como o MVF são equivalentes para uma série de problemas, levando muitas pessoas a confundi-los.

O MDF, foi durante muitos anos, utilizado na solução de problemas de fluidos , mas não se tinha habilidade no tratamento de geometrias complexas, e teve todo o seu desenvolvimento baseado em coordenadas ortogonais, como a cartesiana, a cilíndrica e a esférica. Segundo Maliska (1995), muitas pessoas ainda vinculam o MDF com malhas cartesianas, de forma equivocada, uma vez que esse método pode ser aplicado a qualquer tipo de malha, mesmo as não estruturadas, usualmente utilizadas em elementos finitos. 
O MEF clássico empregava funções peso do tipo Galerkin, que equivale ao uso das diferenças centrais no método das diferenças finitas, para resolver os problemas de escoamento de fluidos. No entanto, observou-se que o método de Galerkin é adequado apenas para problemas puramente difusivos.

Um dos primeiros pesquisadores a aplicar esse método para solução de escoamento foi Zienkiewiscz et al. (1976), com a publicação de um trabalho que aplicava o método de Galerkin, onde as funções de expansão e peso utilizadas eram funções lineares e quadráticas. Na ocasião, o sucesso desse método, deve-se ao fato do problema resolvido ser essencialmente difusivo. Contudo, quando esse método era utilizado para resolver problemas com convecção predominante, eram observados dois problemas básicos. O primeiro é o surgimento de divergências na solução numérica, provocadas pela utilização de um método de solução numérica inadequado para este tipo de problema. O segundo é a obtenção de soluções não realistas, isto é, soluções que apresentavam muitas oscilações numéricas, devido ao fato de que um esquema similar ao de diferenças centrais não possui habilidade para dissipar as perturbações inerentes ao processo de solução, segundo Zienkiewicz et al. (1976).

Para resolver problemas convectivos, passou-se a empregar as funções peso do tipo Petrov-Galerkin. Este tratamento possibilitou um grande avanço na aplicação do MEF, permitindo a solução de problemas de dinâmica de fluidos, com a minimização dos efeitos de difusão numérica. Nesse método, conhecido como SUPG ("Streamline Upwind PetrovGalerkin”), as funções peso que são descontínuas, são construídas adicionando-se às funções peso da formulação de Galerkin uma perturbação que atua somente na direção do escoamento.

Hughes (1987) apresenta um breve histórico do surgimento do método SUPG. O nome Petrov provém da referência fornecida por Mikhlin (1964), embora o crédito desta técnica não se deva a Petrov, enquanto que o nome Galerkin é empregado adequadamente pelo fato de o método SUPG estar baseado na formulação clássica de Galerkin. 
O primeiro trabalho publicado sobre esse assunto foi realizado por Hughes e Brooks (1979), onde o método SUPG foi aplicado às equações de Navier-Stokes para fluidos incompressíveis. Posteriormente, os mesmos autores publicaram outros trabalhos sobre esse método, Brooks e Hughes (1980) e Brooks (1981). Observou-se, na ocasião, que nos trabalhos de Dendy (1974), Wahlbin (1974), Raymond e Garter (1976), Baker (1979) e Argyris et al. (1984), o tratamento dos termos convectivos ou eram desprezados ou tratados de forma incorreta. Esse problema foi completamente resolvido por Hughes e Brooks (1982). A partir desse trabalho surgiram outros como o de Johnson et al (1984), Johson (1982), Näver (1982), Johson e Szepessy (1985), Johson (1986), Johson e Saranen (1986), Johson e Szepessy (1986 a), Johson e Szepessy (1986 b), os quais desenvolveram uma análise matemática extensa do método SUPG. Nesses trabalhos essa metodologia também foi estendida para problemas transitórios e para fluidos incompressíveis. Adicionalmente, Hughes (1987) aplicou o método SUPG para a solução das equações de Navier-Stokes para fluidos compressíveis em duas dimensões.

Atualmente, observa-se que os métodos dos volumes finitos e dos elementos finitos resolvem problemas altamente convectivos em geometrias complexas, mostrando que existem semelhanças entre eles. Do ponto de vista matemático, esses três métodos numéricos (MDF, MVF e MEF) podem ser derivados do método dos resíduos ponderados, empregando-se diferentes funções peso. No caso do MDF, a função peso utilizada é a função delta no ponto considerado. Para o MVF, a função peso empregada é uma constante igual a 1 no volume elementar e igual a zero em todos os outros volumes elementares. No caso do MEF (Petrov-Galerkin) são utilizadas funções peso descontínuas.

Embora os métodos numéricos mencionados sejam todos derivados do mesmo princípio e diferirem apenas na função peso escolhida, na prática o que se tem são diferentes graus de experiência dos usuários, com os diversos métodos na solução de variados tipos de problemas. 
Na medida em que nesta tese é utilizada a formulação Petrov-Galerkin, é realizada uma revisão detalhada dessa classe de método. A partir da década de 80, o método SUPG começou a ser largamente empregado para a solução dos mais diversos tipos de problemas de dinâmica de fluidos e problemas convectivos-difusivos. Algumas das aplicações nessa área podem ser vistas nos trabalhos a seguir.

Peraire et al (1986) elaboraram um procedimento para malhas irregulares com o objetivo de melhorarem a solução de problemas em regime permanente das equações de Euler em 2 dimensões. A técnica é implementada utilizando-se um algoritmo de solução baseado no método dos elementos finitos, com elementos triangulares e aproximação temporal.

Tezduyar et al (1988) empregaram o método SUPG na solução das equações de Navier-Stokes em fluidos incompressíveis. Nesse trabalho, a técnica utilizada para solucionar o problema do acoplamento pressão-velocidade foi resolver as equações da quantidade de movimento em termos de corrente e de vorticidade. Nesse caso, o número de funções é reduzido de três para dois e o campo de velocidade do fluido é calculado indiretamente a partir da integração do campo de vorticidade. Embora essa técnica seja atraente, ela se aplica apenas para problemas bi-dimensionais. Além disso, os autores observaram que o uso dessa técnica tornava a convergência lenta e o processo iterativo bastante instável.

Brueckner e Heinrich (1991) aplicaram o método de Petrov-Galerkin para a solução das equações de Navier-Stokes de escoamentos compressíveis. A solução numérica das equações que governam o movimento de escoamento compressível, em alta velocidade, possui várias dificuldades que provém principalmente da escala de dimensão associada às ondas de choque e às camadas limites viscosas. Além disso, os algoritmos numéricos invariavelmente exibem instabilidades em regiões de grandes mudanças nas variáveis. Portanto, os algoritmos clássicos devem ser modificados para manter a estabilidade e obter soluções fisicamente plausíveis. Nesse contexto, nos métodos de diferenças finitas e 
elementos finitos, essas modificações são conhecidas como método de captura de choque e são essencialmente de três tipos:

- métodos de viscosidade artificial representados pelos métodos de Von Neumann e Richtmeyer (1950), Lapidus (1967) e Zienkiewicz et al (1985) e pelos métodos de Petrov-Galerkin desenvolvidos por Hughes e Tezduyar (1984);

- métodos de Transporte de Fluxo Corrigido (FCT) desenvolvidos por Boris e Book (1973) e Zalezak (1979); e

- método de Godunov (1959).

Cada um desses métodos tem vantagens e desvantagens segundo Woodward e Colella (1984). O método de Godunov requer uma inversão de Riemann e não é adequado para cálculos multi-dimensionais. Métodos baseados no FCT são difíceis de serem usados no espaço bi-dimensional. Os métodos de viscosidade artificial introduzem parâmetros sem critério de escolha, no entanto, esses métodos são mais simples de serem usados em elementos finitos. O algoritmo de maior sucesso atualmente é baseado nas idéias de Donea (1984) e Zienkiewicz (1985) sob o nome de Taylor-Galerkin, combinado com malha não estruturada. Porém, esse método requer a introdução de uma viscosidade artificial que ainda não tem sido totalmente resolvida.

Baumann et al (1992) desenvolveram uma técnica para acelerar a convergência para soluções estacionárias quando a formulação SUPG é usada. Nesse trabalho é apresentada uma formulação capaz de tratar o domínio de solução com geometrias complexas e usar condições de contorno físicas apropriadas, juntamente com uma taxa de convergência apropriada para a solução estacionária. Essa técnica possui as seguintes características: formulação tipo SUPG com um operador de captura de choque, refinamento adaptivo da malha, integração explícita com um passo de tempo local e expansão de Taylor truncada, usada na formulação residual ponderada. 
Aliabadi e Tezduyar (1993) apresentaram uma formulação tempo-espaço aplicável em domínios deformáveis no tempo para simulação de escoamentos compressíveis viscosos. A técnica empregada é o método SUPG modificado. A formulação de elementos finitos das equações de conservação é escrita sobre o domínio espaço-tempo do problema e, portanto, a deformação do domínio espacial com respeito ao tempo é levada em consideração automaticamente. A frequência de "remeshing” é minimizada para diminuir os erros envolvidos. É demonstrado também que a combinação do método SUPG com a aproximação espaço-tempo tem capacidade de manipular problemas de escoamento compressíveis complexos, incluindo aqueles com movimento de superfícies.

Almeida e Galeão (1994) desenvolveram uma formulação que utiliza o método dos elementos finitos com função peso tipo Petrov-Galerkin combinada com um refinamento adaptivo de malha. Essa formulação tem sido apropriada para resolver problemas que apresentam ondas de choque e camadas limites, por permitir um alto refinamento na malha nestas regiões do escoamento, onde as variáveis variam fortemente.

Nigro et al (1994) apresentaram um trabalho que descreve um algoritmo para modelar escoamento de líquido em um recipiente com gás, com especial atenção para a determinação do campo de velocidades. A discretização é baseada no método SUPG. O conceito do SUPG é usado para estabilizar não somente os termos de advecção mas também o operador sônico cuja estrutura complexa é responsável pela condição de incompressibilidade. Um termo de estabilização proporcional ao Laplaciano da pressão na equação da continuidade e outro idêntico à viscosidade do meio são gerados de forma a manter a consistência das equações.

Chittur e Venkatasubban (1995) apresentaram uma nova formulação para o ALE “Arbitrary Lagrangian Eulerian Equations” descrevendo um escoamento de fluido compressível em termos de um sistema de malhas em movimento. Uma importante consequência dessa formulação é a eliminacão dos termos envolvendo a dilatação da rede. Esse tipo de aproximação tem levado ao desenvolvimento de métodos computacionais robustos para o tratamento da mecânica dos fluidos multi-corpo. 
Barsoum e Alexandrou (1995) apresentaram uma aproximação estável para resolver escoamentos com convecção dominante na presença de efeitos de compressibilidade. Essa aproximação emprega as funções peso tipo Petrov-Galerkin para as equações que governam o movimento e é utilizada com sucesso em problemas de escoamento com alto número de Péclet. A estabilidade dos algoritmos limitam a capacidade de modelar problemas com convecção dominante, onde o número de Péclet é alto. Os métodos de diferenças finitas tratam esse problema com "upstream differencing” para os termos convectivos e aproximação por diferença central para os termos difusivos. Observase que, até recentemente, antes do advento da formulação Petrov-Galerkin, os métodos de elementos finitos não eram hábeis para modelar escoamentos com alto número de Péclet. Nesse trabalho aplica-se o método dos elementos finitos utilizando funções de expansão lineares, que é semelhante à diferença central no espaço e à diferença para a frente no tempo, no método das diferenças finitas.

Aliabadi e Tezduyar (1995) apresentaram uma formulação de elementos finitos baseada em variáveis de conservação utilizando o método SUPG. O cálculo é baseado em um método semi-implícito sobre uma malha não estruturada. Os problemas de escoamento com contorno fixo são resolvidos com uma formulação semidiscreta e os problemas de escoamento com contorno móvel são resolvidos com uma formulação estabilizada no espaço e tempo, com domínio espacial deformável. A formulação semidiscreta considera um domínio espacial fixo dividido em sub-domínios. As funções de expansão utilizadas são polinômios de primeira ordem.

Sampaio e Moreira (1995) apresentaram um programa para a simulação de escoamento compressível e quase incompressível, em duas dimensões. A aproximação espacial é feita utilizando o método dos elementos finitos e funções de peso PetrovGalerkin. Essa formulação introduz termos estabilizadores da pressão que permitem a simulação de escoamentos a baixos números de Mach sem necessidade de um modelo incompressível. 
Storti et al (1996) elaboraram uma nova formulação SUPG para a solução das equações de Navier-Stokes para fluidos compressíveis e próximo do incompressível. Esse método satisfaz sistemas do tipo advectivo-difusivo unidimensional e sistemas multidimensionais com advecção predominante. A formulação numérica é baseada no método dos resíduos ponderados aplicando Petrov-Galerkin, onde se permite testar várias funções não necessariamente contínuas. Esse método introduz uma dissipação numérica, que é necessária para estabilizar a solução em caso de problemas de advecção predominante.

Nigro et al (1998) estudaram o desempenho de um programa computacional 3-D, desenvolvido em elementos finitos, cujo objetivo era mostrar a capacidade do programa em lidar com grandes faixas de problema, isto é, escoamento compressível e próximo do incompressível que utiliza as equações de Euler ou de Navier-Stokes. O programa utiliza as hipóteses do fluido Newtoniano e escoamento laminar. No início os métodos baseados no operador “splitting” e “perturbation” eram preferidos, porém, com o uso dos algoritmos que marcham no tempo, as matrizes de massa de pré-condicionamento (PMM) tornaram-se mais populares. Com esse tipo de esquema de relaxação foi possível acelerar a convergência para soluções do estado estacionário e obter bons resultados.

Sabundjian e Cabral (1999) aplicaram o método da expansão em funções hierárquicas na solução das equações de Navier-Stokes em duas dimensões, para fluidos incompressíveis em regime laminar. Nesse trabalho utilizou-se, pela primeira vez, esse método de elementos finitos com a formulação de Petrov-Galerkin, em conjunto com a expansão das variáveis em funções hierárquicas nas equações de Navier-Stokes. O tratamento dos diversos termos das equações de conservação foi feito de forma distinta, ou seja, para os termos convectivos das equações de Navier-Stokes foram utilizados a formulação Petrov-Galerkin, enquanto que para os termos temporais, viscosos e difusivos foram empregados a formulação de Galerkin. Os resultados obtidos nesse trabalho mostraram que o método de expansão em funções hierárquicas foi aplicado com sucesso na solução de problemas em mecânica dos fluidos, quando envolve fluidos incompressíveis em regime laminar. 
Xikui et al (2000) utilizaram a formulação de Galerkin para o método dos elementos finitos com um algoritmo implícito para a solução das equações de difusãoconvecção, multidimensional, dependente do tempo. O método é formulado com base na combinação de precisão com o procedimento de integração numérica implícita. O procedimento de integração preciso, com um algoritmo $2^{\mathrm{N}}$, é tomado como ferramenta para determinar a derivada material (Lagrangiana) da função convectiva. Os resultados mostram um bom desempenho do método em estabilidade e precisão dos resultados obtidos.

Wong et al (2001) apresentaram o algoritmo SUPG para a equação de Euler em escoamentos compressíveis para baixos números de Mach. As equações de Euler são escritas em termos das variáveis de entropia, que resultam em matrizes Jacobianas simétricas. Tem-se que, para baixos números de Mach, o método SUPG com escolhas padrões para a estabilização das matrizes falha em prover estabilização adequada. Isso resulta em uma degradação da precisão da solução. Portanto, esse trabalho, propõe uma matriz de estabilização na qual incorpora argumentos adimensionais e exibe comportamento apropriado para baixos números de Mach. A matriz de estabilização é feita com um conjunto de variáveis que convergem para números de Mach que tendem a zero. O algoritmo resultante é capaz de calcular com precisão escoamentos com número de Mach da ordem de $10^{-3}$.

Willis et al (2004) apresentaram o método do elemento de contorno de Galerkin (BEM) para a solução das equações integrais em três dimensões. O método incorpora nós baseado em funções de formato linear, de uma ou duas camadas sobre elementos triangulares planos. A solução do método é acelerada usando um algoritmo pré-corretor de transformada de Fourier (pFFT). O método é aplicado a problemas de escoamento ao redor de esferas e de asas de avião. Os resultados obtidos foram comparados com as soluções analíticas e com resultados 2-D da literatura, mostrando-se bastante precisos. 
Akin e Tezduyar (2004) apresentaram, para elementos lineares e de ordem superior, várias maneiras de calcular o limite advectivo do parâmetro de estabilização usado na formulação SUPG para problemas de escoamento. Nesse estudo foram comparados diversas formulações, sendo que a formulação elemento matriz resultou no menor valor do parâmetro de estabilização e que o valor do parâmetro diminui com o aumento do grau do polinômio.

Ghorbany e Soheili (2004) estudaram os métodos de movimento sem malha, que formam a nova geração dos métodos numéricos para as equações diferenciais parciais dependentes do tempo, aplicados à regiões de choque ou de alto gradiente. Esses métodos estão acoplados ao método dos elementos finitos e suas coordenadas são as incógnitas dependentes do tempo. Um termo adicional de energia é adicionado ao método para previnir altas velocidades, colapsamentos de nós e de concentração de nós em regiões de choque.

Okusanya et al (2004) descreveram um método multimalha para a solução de discretizações em elementos finitos das equações de Euler e Navier Stokes, sobre malhas generalizadas não estruturadas. Os termos viscosos são discretizados de maneira consistente sobre malhas pouco refinadas usando a formulação de Galerkin. A diminuição da estabilidade dos operadores não viscosos, devido à combinação dos operadores de alta ordem com malhas pouco refinadas, é compensada por um esquema de relaxação implícito. A taxa de convergência mostra-se boa e não é afetada pelo tamanho da célula.

Zungjun e Gecheng (2005) utilizaram pela primeira vez um esquema de diferenças para frente, de baixa difusão, para calcular escoamentos 3-D de sistemas de propulsão. O esquema chamado de E-CUSP contém a energia total no vetor convectivo e é totalmente consistente com as direções características. Nesse trabalho, simulou-se bocais transônicos de seções circulares e retas, e dutos com ondas de choque. O esquema mostrou-se bastante eficiente para cálculos 3-D. 
Tejada-Martinez e Jansen (2005) investigaram os papéis das modelagens físicas e numéricas em escala submalha. As escalas submalhas foram representadas por um modelo físico de simulação conhecido pelo nome de "Smagorinsky", ou simplesmente por modelo dinâmico, e por um modelo numérico conhecido por SUPG. Estudou-se a interação entre os modelos físicos e numéricos analisando-se a dissipação de energia associada a eles. Baseado nesse estudo, sugeriu-se uma modificação no modelo físico para descontar a dissipação numérica do método. O modelo físico modificado mostrou-se muito preciso para escoamentos turbulentos em canais. 


\section{3 - EQUAÇÕES DE CONSERVAÇÃO}

As equações que governam a dinâmica dos fluidos são as equações de conservação da massa (continuidade), de conservação da quantidade de movimento (equações de Navier-Stokes) e de conservação da energia. Essas equações representam matematicamente os três princípios físicos fundamentais que são, respectivamente, o princípio de conservação da massa, a segunda lei de Newton e a lei de conservação da energia, nos quais toda a dinâmica dos fluidos está fundamentada. Esse conjunto de equações encontra-se descrito neste capítulo.

\section{1 - Equação de conservação da massa (continuidade)}

A equação de conservação da massa, escrita para um sistema cartesiano de coordenadas em três dimensões, segundo Bejan (1984), é dada por:

$$
\frac{\partial \rho}{\partial t}+\frac{\partial(\rho u)}{\partial x}+\frac{\partial(\rho v)}{\partial y}+\frac{\partial(\rho w)}{\partial z}=0
$$

onde $\rho$ é a massa específica do fluido, $t$ é o tempo, $u, v e w$ são as velocidades nas direções $x, y$ e $z$, respectivamente.

\section{2 - Equação de conservação da quantidade de movimento}

A partir da $2^{\underline{a}}$ lei de Newton, pode-se escrever as equações de conservação da quantidade de movimento para cada uma das três direções do sistema cartesiano de coordenadas. Para tanto, utiliza-se a Figura 3.1, na qual se observam as forças que agem sobre um volume elementar de fluido em cada uma das direções. Essas forças são as seguintes: 
a) forças de campo: forças gravitacionais e eletromagnéticas (forças que agem por distância); e

b) forças superficiais: forças que agem na superfície do volume elementar, forças normais ou tangenciais.

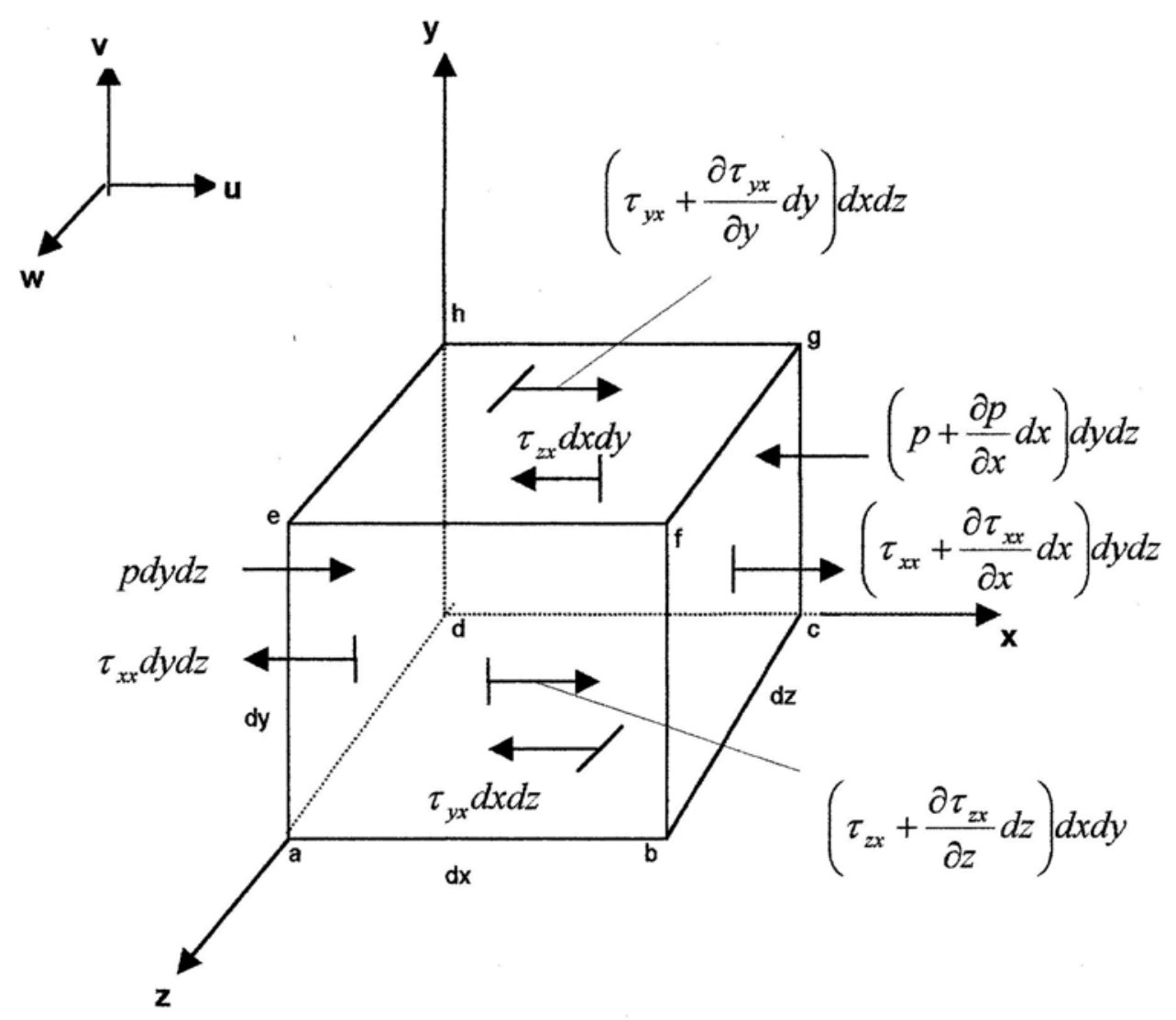

Figura 3.1 - Elemento de fluido em movimento.

A forma genérica das equações de conservação da quantidade de movimento, segundo Anderson et al. (1984), para cada uma das três direções é dada por:

$$
\frac{\partial U}{\partial t}+\frac{\partial F}{\partial x}+\frac{\partial G}{\partial y}+\frac{\partial H}{\partial z}=J
$$


A equação (3-2) é conhecida como sendo a forma conservativa das equações do movimento, onde $U, F, G, H$ e $J$ são representados pelos seguintes vetores:

$$
U=\left[\begin{array}{c}
\rho u \\
\rho v \\
\rho w
\end{array}\right]
$$

$$
F=\left[\begin{array}{l}
\rho u^{2}+p-\tau_{x x} \\
\rho v u-\tau_{x y} \\
\rho w u-\tau_{x z}
\end{array}\right] ;
$$

$$
G=\left[\begin{array}{l}
\rho u v-\tau_{y x} \\
\rho v^{2}+p-\tau_{y y} \\
\rho w v-\tau_{y z}
\end{array}\right] ;
$$

$$
H=\left[\begin{array}{lll}
\rho u w & -\tau_{z x} & \\
\rho v w-\tau_{z y} & \\
\rho w^{2}+p & - & \tau_{z z}
\end{array}\right]
$$

e

$$
J=\left[\begin{array}{c}
\rho f_{x} \\
\rho f_{y} \\
\rho f_{z}
\end{array}\right]
$$

onde $p$ é a pressão, $f_{x}, f_{y}$ e $f_{z}$ são as forças de campo, $\tau_{x x}, \tau_{x y}, \tau_{x z}, \tau_{y x}, \tau_{y y}, \tau_{y z}, \tau_{z x}, \tau_{z y}$ e $\tau_{z z}$ são as tensões de cisalhamento, em cada um dos planos. 
Assume-se que o segundo coeficiente de viscosidade média $\left(\mu\right.$ ') é igual a $-\frac{2}{3}$ da viscosidade dinâmica $(\mu)$. Portanto, as tensões de cisalhamento que aparecem nas equações (3-4), (3-5) e (3-6) são representadas por:

$$
\begin{aligned}
& \tau_{x x}=\frac{2}{3} \mu\left(2 \frac{\partial u}{\partial x}-\frac{\partial v}{\partial y}-\frac{\partial w}{\partial z}\right) \\
& \tau_{y y}=\frac{2}{3} \mu\left(2 \frac{\partial v}{\partial y}-\frac{\partial u}{\partial x}-\frac{\partial w}{\partial z}\right) ; \\
& \tau_{z z}=\frac{2}{3} \mu\left(2 \frac{\partial w}{\partial z}-\frac{\partial u}{\partial x}-\frac{\partial v}{\partial y}\right) ; \\
& \tau_{x y}=\mu\left(\frac{\partial u}{\partial y}+\frac{\partial v}{\partial x}\right)=\tau_{y x} \\
& \tau_{x z}=\mu\left(\frac{\partial w}{\partial x}+\frac{\partial u}{\partial z}\right)=\tau_{z x} \\
& \tau_{y z}=\mu\left(\frac{\partial v}{\partial z}+\frac{\partial w}{\partial y}\right)=\tau_{z y} .
\end{aligned}
$$

Nota-se que devido à continuidade do meio demonstra-se que as tensões de cisalhamento são iguais no caso em que os índices são iguais, ou seja, $\tau_{x y}=\tau_{y x}, \tau_{x z}=\tau_{z x} \mathrm{e}$ $\tau_{y z}=\tau_{z y}$

A viscosidade dinâmica neste trabalho é considerada constante. Além disso, na medida em que a massa específica do fluido compressível é baixa, não se-considera nesse trabalho as forças de campo, portanto, o termo fonte representado pelo vetor $J$ é nulo. 
A partir da equação (3-2), considerando-se as hipóteses assumidas, e lembrando que o fluido é compressível, obtêm-se as equações de conservação da quantidade de movimento para cada uma das direções $x, y$ e $z$, respectivamente:

$$
\begin{aligned}
& \frac{\partial \rho u}{\partial t}+\frac{\partial(\rho u u)}{\partial x}+\frac{\partial(\rho u v)}{\partial y}+\frac{\partial(\rho u w)}{\partial z}=-\frac{\partial p}{\partial x}+\frac{4}{3} \mu \frac{\partial^{2} u}{\partial x^{2}}+\mu \frac{\partial^{2} u}{\partial y^{2}}+\mu \frac{\partial^{2} u}{\partial z^{2}} \\
& \frac{\partial \rho v}{\partial t}+\frac{\partial(\rho v u)}{\partial x}+\frac{\partial(\rho v v)}{\partial y}+\frac{\partial(\rho v w)}{\partial z}=-\frac{\partial p}{\partial y}+\mu \frac{\partial^{2} v}{\partial x^{2}}+\frac{4}{3} \mu \frac{\partial^{2} v}{\partial y^{2}}+\mu \frac{\partial^{2} v}{\partial z^{2}}
\end{aligned}
$$

$\mathrm{e}$

$$
\frac{\partial \rho w}{\partial t}+\frac{\partial(\rho w u)}{\partial x}+\frac{\partial(\rho w v)}{\partial y}+\frac{\partial(\rho w w)}{\partial z}=-\frac{\partial p}{\partial z}+\mu \frac{\partial^{2} w}{\partial x^{2}}+\mu \frac{\partial^{2} w}{\partial y^{2}}+\frac{4}{3} \mu \frac{\partial^{2} w}{\partial z^{2}}
$$

Define-se a variável velocidade mássica em cada uma das direções $x, y$ e $z$, como sendo:

$$
\begin{aligned}
& G_{x}=\rho u ; \\
& G_{y}=\rho v ;
\end{aligned}
$$

$\mathrm{e}$

$$
G_{z}=\rho w .
$$

Substituindo-se as equações (3-17), (3-18) e (3-19) nas equações (3-14), (3-15) e (316), respectivamente, a fim de simplificá-las e obter as novas equações de conservação da quantidade de movimento:

$$
\frac{\partial G_{x}}{\partial t}+\frac{\partial\left(G_{x} u\right)}{\partial x}+\frac{\partial\left(G_{x} v\right)}{\partial y}+\frac{\partial\left(G_{x} w\right)}{\partial z}=-\frac{\partial p}{\partial x}+\frac{4}{3} \mu \frac{\partial^{2} u}{\partial x^{2}}+\mu \frac{\partial^{2} u}{\partial y^{2}}+\mu \frac{\partial^{2} u}{\partial z^{2}}
$$




$$
\frac{\partial G_{y}}{\partial t}+\frac{\partial\left(G_{y} u\right)}{\partial x}+\frac{\partial\left(G_{y} v\right)}{\partial y}+\frac{\partial\left(G_{y} w\right)}{\partial z}=-\frac{\partial p}{\partial y}+\mu \frac{\partial^{2} v}{\partial x^{2}}+\frac{4}{3} \mu \frac{\partial^{2} v}{\partial y^{2}}+\mu \frac{\partial^{2} v}{\partial z^{2}}
$$

e

$$
\frac{\partial G_{z}}{\partial t}+\frac{\partial\left(G_{z} u\right)}{\partial x}+\frac{\partial\left(G_{z} v\right)}{\partial y}+\frac{\partial\left(G_{z} w\right)}{\partial z}=-\frac{\partial p}{\partial z}+\mu \frac{\partial^{2} w}{\partial x^{2}}+\mu \frac{\partial^{2} w}{\partial y^{2}}+\frac{4}{3} \mu \frac{\partial^{2} w}{\partial z^{2}}
$$

\section{3 - Equação de conservação da energia}

Aplicando-se o $1^{0}$ princípio da termodinâmica, em um volume elementar de fluido, obtém-se a equação de conservação da energia para um sistema de coordenadas cartesianas em três dimensões que, segundo Anderson et al. (1984), é dada por:

$$
\frac{\partial(\rho e)}{\partial t}+\frac{\partial(\rho e u)}{\partial x}+\frac{\partial(\rho e v)}{\partial y}+\frac{\partial(\rho w e)}{\partial z}=\frac{\partial Q}{\partial t}+K \nabla^{2} T-p \operatorname{div} \vec{V}+\phi,
$$

onde $e$ é a energia interna específica, $K$ é a condutividade térmica (que neste trabalho é assumida como sendo constante), $T$ é a temperatura, $Q$ é a geração de energia interna por unidade de volume e $\phi$ é a função de dissipação, que é definida pela seguinte expressão:

$$
\begin{aligned}
\phi= & \mu\left[2\left(\frac{\partial u}{\partial x}\right)^{2}+2\left(\frac{\partial v}{\partial y}\right)^{2}+2\left(\frac{\partial w}{\partial z}\right)^{2}+\left(\frac{\partial v}{\partial x}+\frac{\partial u}{\partial y}\right)^{2}+\left(\frac{\partial w}{\partial y}+\frac{\partial v}{\partial z}\right)^{2}+\right. \\
& \left.+\left(\frac{\partial u}{\partial z}+\frac{\partial w}{\partial x}\right)^{2}-\frac{2}{3}\left(\frac{\partial u}{\partial x}+\frac{\partial v}{\partial y}+\frac{\partial w}{\partial z}\right)\right]
\end{aligned}
$$

Para o caso de gases perfeitos, pode-se reescrever a equação (3-23) em termos da energia interna por unidade de volume, $E$, ou seja: 


$$
\begin{aligned}
& \frac{\partial E}{\partial t}+\frac{\partial(E u)}{\partial x}+\frac{\partial(E v)}{\partial y}+\frac{\partial(E w)}{\partial z}=\frac{\partial Q}{\partial t}+K \nabla^{2} T-p \operatorname{div} \vec{V}+\mu\left[2\left(\frac{\partial u}{\partial x}\right)^{2}+2\left(\frac{\partial v}{\partial y}\right)^{2}+\right. \\
& \left.+2\left(\frac{\partial w}{\partial z}\right)^{2}+\left(\frac{\partial v}{\partial x}+\frac{\partial u}{\partial y}\right)^{2}+\left(\frac{\partial w}{\partial y}+\frac{\partial v}{\partial z}\right)^{2}+\left(\frac{\partial u}{\partial z}+\frac{\partial w}{\partial x}\right)^{2}-\frac{2}{3}\left(\frac{\partial u}{\partial x}+\frac{\partial v}{\partial y}+\frac{\partial w}{\partial z}\right)\right]
\end{aligned}
$$

onde assume-se que não há geração de energia interna, ou seja, $Q$ é igual a zero e $E$ é dado por

$$
E=\rho e .
$$

O cálculo da temperatura é feito a partir da definição de calor específico a volume constante para gases perfeitos, que é dada por:

$$
\left.\frac{\partial e}{\partial T}\right|_{V}=c_{V},
$$

onde $c_{v}$ é o calor específico a volume constante. Assumindo $c_{v}$ constante e integrando a equação (3-27), obtém-se a seguinte expressão:

$$
e-e_{0}=c_{v}\left(T-T_{0}\right) \text {. }
$$

Assumindo-se que o estado “0” é um estado de referência, no qual a energia é igual a zero, a equação anterior é reescrita da seguinte forma:

$$
e=c_{V} T
$$

Colocando a temperatura em termos da energia interna por unidade de volume, obtém-se a seguinte equação: 


$$
E=\rho c_{V} T \text {. }
$$

\section{4 - Equação de estado}

Assumindo que o fluido compressível é um gás perfeito, a sua equação de estado é dada pela equação universal dos gases perfeitos, ou seja:

$$
p=\rho R T
$$

onde $R$ é a constante do gás.

\section{5 - Equações em duas dimensões}

O desenvolvimento matemático do método de expansão em funções hierárquicas, proposto neste trabalho, envolve as equações de conservação da massa, as equações de conservação da quantidade de movimento, as equações de conservação da energia e as equações da velocidade mássica em duas dimensões, além da equação de estado e da equação da energia interna.

A partir da equação (3-1) obtém-se a equação de conservação da massa em duas dimensões, que é dada por:

$$
\frac{\partial \rho}{\partial t}+\frac{\partial \rho u}{\partial x}+\frac{\partial \rho w}{\partial z}=0
$$

As equações de conservação da quantidade de movimento originam-se das equações (3-20) e (3-22), que para as coordenadas cartesianas $x$ e $z$ são, respectivamente:

$$
\frac{\partial G_{x}}{\partial t}+\frac{\partial G_{x} u}{\partial x}+\frac{\partial G_{x} w}{\partial z}=-\frac{\partial p}{\partial x}+\frac{4}{3} \mu \frac{\partial^{2} u}{\partial x^{2}}+\mu \frac{\partial^{2} u}{\partial z^{2}}
$$


e

$$
\frac{\partial G_{z}}{\partial t}+\frac{\partial G_{z} u}{\partial x}+\frac{\partial G_{z} w}{\partial z}=-\frac{\partial p}{\partial z}+\mu \frac{\partial^{2} w}{\partial x^{2}}+\frac{4}{3} \mu \frac{\partial^{2} w}{\partial z^{2}}
$$

As equações da velocidade mássica para as coordenadas cartesianas $x$ e $z$ são, respectivamente:

$$
G_{x}=\rho u
$$

e

$$
G_{z}=\rho w .
$$

Reduzindo-se a equação (3-25) de três para duas dimensões, obtém-se a equação de conservação da energia nas direções $x$ e $z$, que é dada por:

$$
\begin{aligned}
\frac{\partial E}{\partial t}+\frac{\partial E u}{\partial x}+\frac{\partial E w}{\partial z} & =k \nabla^{2} T-p\left(\frac{\partial u}{\partial x}+\frac{\partial w}{\partial z}\right)+\mu\left(\frac{\partial u}{\partial z}+\frac{\partial w}{\partial x}\right)^{2}+ \\
+ & 2 \mu\left[\left(\frac{\partial u}{\partial x}\right)^{2}+\left(\frac{\partial w}{\partial z}\right)^{2}+\frac{\partial u}{\partial x} \frac{\partial w}{\partial z}\right]-\frac{2 \mu}{3}\left(\frac{\partial u}{\partial x}+\frac{\partial w}{\partial z}\right)
\end{aligned}
$$

As outras equações, equação de estado e equação da energia interna, não se alteram ao se passar de três para duas dimensões. 


\section{4 - DESENVOLVIMENTO TEÓRICO}

O método numérico proposto, neste trabalho, consiste em uma aplicação do método da expansão em funções hierárquicas, para a solução de problemas de escoamento em duas dimensões, de fluidos compressíveis em alta velocidade. No desenvolvimento deste trabalho são utilizadas as equações da continuidade, de conservação da quantidade de movimento, da velocidade mássica, de estado, de conservação da energia e da temperatura, conforme o desenvolvimento realizado no capítulo anterior e cujas equações em duas dimensões são mostradas resumidamente a seguir.

Equação da continuidade:

$$
\frac{\partial \rho}{\partial t}+\frac{\partial \rho u}{\partial x}+\frac{\partial \rho w}{\partial z}=0
$$

Equações de conservação da quantidade de movimento:

$$
\frac{\partial G_{x}}{\partial t}+\frac{\partial G_{x} u}{\partial x}+\frac{\partial G_{x} w}{\partial z}=-\frac{\partial p}{\partial x}+\frac{4}{3} \mu \frac{\partial^{2} u}{\partial x^{2}}+\mu \frac{\partial^{2} u}{\partial z^{2}}
$$

$\mathrm{e}$

$$
\frac{\partial G_{z}}{\partial t}+\frac{\partial G_{z} u}{\partial x}+\frac{\partial G_{z} w}{\partial z}=-\frac{\partial p}{\partial z}+\mu \frac{\partial^{2} w}{\partial x^{2}}+\frac{4}{3} \mu \frac{\partial^{2} w}{\partial z^{2}}
$$

Equações das velocidades mássicas:

$$
G_{x}=\rho u
$$

$\mathrm{e}$

$$
G_{z}=\rho w
$$


Equação de estado:

$$
p-\rho R T=0 .
$$

Equação de conservação da energia:

$$
\begin{aligned}
& \frac{\partial E}{\partial t}+\frac{\partial E u}{\partial x}+\frac{\partial E w}{\partial z}=k \nabla^{2} T-p\left(\frac{\partial u}{\partial x}+\frac{\partial w}{\partial z}\right)+\mu\left(\frac{\partial u}{\partial z}+\frac{\partial w}{\partial x}\right)^{2}+ \\
&+2 \mu\left[\left(\frac{\partial u}{\partial x}\right)^{2}+\left(\frac{\partial w}{\partial z}\right)^{2}+\frac{\partial u}{\partial x} \frac{\partial w}{\partial z}\right]-\frac{2 \mu}{3}\left(\frac{\partial u}{\partial x}+\frac{\partial w}{\partial z}\right) .
\end{aligned}
$$

Equação da temperatura:

$$
E=\rho c_{v} T .
$$

Neste trabalho é utilizada a formulação de Petrov-Galerkin consistente na solução das equações de Navier-Stokes, sendo que as funções de expansão utilizadas são baseadas em polinômios de Legendre, ajustadas nos elementos retangulares de uma forma conveniente.

A seguir são apresentados todos os passos do desenvolvimento matemático aplicado ao conjunto de equações de conservação e de estado, equações (4-1) à (4-8), para resolver problemas que envolvam fluidos compressíveis em alta velocidade em duas dimensões. $\mathrm{O}$ primeiro passo é integrar e pesar esse conjunto de equações em cada elemento. A função peso utilizada em todos os termos das equações de conservação, de estado e algébricas, segue a formulação Petrov-Galerkin, conhecida como formulação Petrov-Galerkin consistente. A partir dessa manipulação matemática, obtém-se, então, um conjunto de equações cuja solução apresenta o campo de velocidades, pressão e temperatura do fluido. 
Observa-se que os termos convectivos das equações de conservação são idênticos, a menos das variáveis envolvidas, isto facilita a solução numérica das equações.

\section{1 - Discretização do domínio de solução}

Como mencionado anteriormente, este trabalho utiliza malhas cartesianas estruturadas para a discretização do domínio da solução. A Figura 4.1 apresenta um esquema de uma malha retangular estruturada, da forma como é utilizada nesta tese.

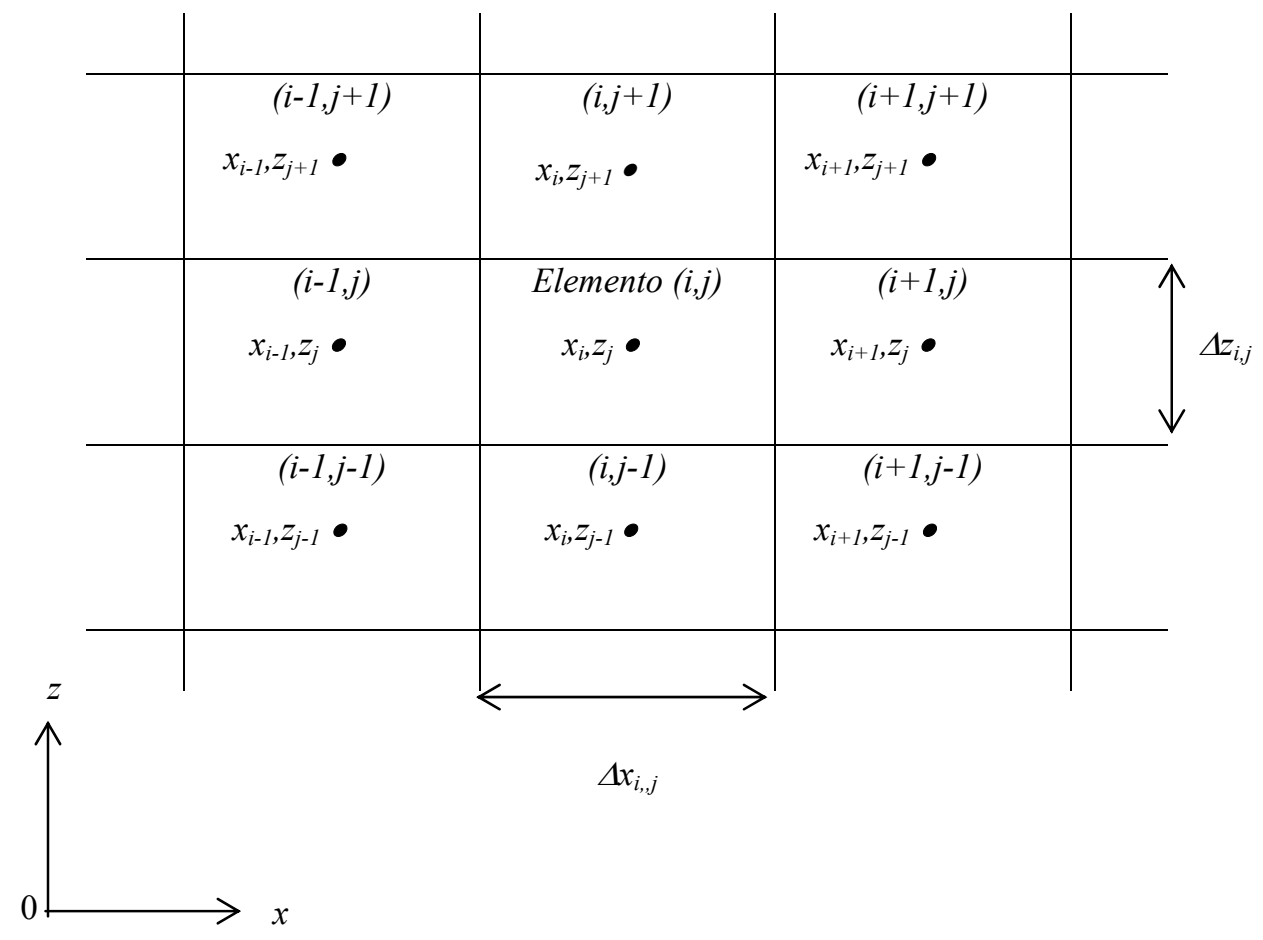

Figura 4.1 - Esquema da malha retangular utilizada.

Na malha da Figura 4.1, $\Delta x_{i, j}$ é o espaçamento na direção $x$ do elemento $i, j, \Delta z_{i, j}$ é o espaçamento na direção $z$ do elemento $i, j$ e as coordenadas $x_{i, j}$ e $z_{i, j}$ representam o ponto central do elemento $i, j$.

Para facilitar a solução das equações de conservação e, posteriormente, permitir o uso de geometrias irregulares, sem grandes modificações nas equações, é utilizado um 
outro sistema de coordenadas. Nesse novo sistema, as coordenadas são as variáveis $\xi$ e $\eta$, cuja correspondência com as coordenadas do sistema cartesiano $x$ e $z$, de cada elemento, é dada por:

$$
\xi=2 \frac{\left(x-x_{i, j}\right)}{\Delta x_{i, j}}
$$

e

$$
\eta=2 \frac{\left(z-z_{i, j}\right)}{\Delta z_{i, j}} .
$$

Observa-se que tanto $\xi$ como $\eta$ variam de -1 a 1 dentro do elemento $i, j$. Nota-se que as coordenadas $\xi$ e $\eta$ são coordenadas locais de cada elemento.

As derivadas das coordenadas $\xi$ e $\eta$ em função das coordenadas cartesianas $x$ e $z$ são obtidas pela derivação das equações (4-9) e (4-10), respectivamente, obtendo-se o seguinte:

$$
\frac{\partial \xi}{\partial x}=\frac{2}{\Delta x_{i, j}} ;
$$

e

$$
\frac{\partial \eta}{\partial \mathrm{z}}=\frac{2}{\Delta \mathrm{z}_{\mathrm{i}, \mathrm{j}}} .
$$

As variáveis $\rho, u, w, G_{x}, G_{z}, E, p$ e $T$ são descritas por uma série de funções dentro de cada um dos elementos $i, j$ do domínio de discretização, como se segue: 


$$
\begin{aligned}
& \rho=\sum_{\mathrm{m}=1}^{\mathrm{M}} \rho_{\mathrm{m}} \mathrm{N}_{m} ; \quad \mathrm{u}=\sum_{\mathrm{m}=1}^{\mathrm{M}} \mathrm{u}_{\mathrm{m}} \mathrm{N}_{m} ; \quad \mathrm{w}=\sum_{\mathrm{m}=1}^{\mathrm{M}} \mathrm{W}_{\mathrm{m}} \mathrm{N}_{m} ; \quad \mathrm{G}_{\mathrm{x}}=\sum_{\mathrm{m}=1}^{\mathrm{M}} \mathrm{G}_{\mathrm{xm}} \mathrm{N}_{m} ; \\
& \mathrm{G}_{\mathrm{z}}=\sum_{m=1}^{\mathrm{M}} \mathrm{G}_{z \mathrm{~m}} \mathrm{~N}_{m} ; \mathrm{p}=\sum_{\mathrm{m}=1}^{\mathrm{M}} \mathrm{p}_{\mathrm{m}} \mathrm{N}_{m} ; \mathrm{E}=\sum_{m=1}^{\mathrm{M}} \mathrm{E}_{\mathrm{m}} \mathrm{N}_{m} ; \mathrm{e} \quad \mathrm{T}=\sum_{m=1}^{\mathrm{M}} \mathrm{T}_{\mathrm{m}} \mathrm{N}_{m} .
\end{aligned}
$$

onde $M$ é o número total de funções utilizadas na expansão em série das variáveis, $N_{m}$ é a $m$-ésima função de expansão para o elemento $i, j$ e $\rho_{m}^{i, j}, u_{m}^{i, j}, w_{m}^{i, j}, G_{x m}^{i, j}, G_{z m}^{i, j}, E_{m}^{i, j}, p_{m}^{i, j} e T_{m}^{i, j}$ são, respectivamente, os coeficientes das variáveis $\rho, u, w, G_{x}, G_{z}, E, p$ e $T$, correspondentes à $m$-ésima função de expansão para o elemento $i, j$ da malha. Ressalta-se que, dependendo do grau da expansão ou do número de funções de expansão utilizadas nas séries, pode-se ajustar a precisão desejada para a solução. Uma descrição das funções de expansão é fornecida no item 4.3.

\section{2 - Desenvolvimento matemático das equações de conservação para um elemento}

As equações utilizadas para calcular o escoamento desejado são obtidas pela integração ponderada das equações (4-1) à (4-8), como é apresentado no desenvolvimento que se segue.

Pesando-se e integrando-se a equação da conservação de massa no elemento $i, j$, equação (4-1), obtém-se a seguinte expressão:

$$
\int_{V} P_{m} \frac{\partial \rho}{\partial t} d V+\int_{V} P_{m} \frac{\partial \rho u}{\partial x} d V+\int_{V} P_{m} \frac{\partial \rho w}{\partial z} d V=0, \text { para } m=1, \ldots, M,
$$

onde $V$ é o volume do elemento $i, j, P_{m}$ é a $m$-ésima função peso associada à $m$-ésima função de expansão no elemento $i, j$. Observa-se que essa equação representa um sistema de $M$ equações para o elemento $i, j$. Sendo que a $m$-ésima equação corresponde ao $m$-ésimo coeficiente da expansão da correspondente variável ou da $m$-ésima função de expansão. 
Aproxima-se a derivada temporal, presente na equação anterior, por uma diferença para trás e obtém-se a seguinte equação:

$$
\int_{V} P_{m} \frac{\rho-\rho^{t-\Delta t}}{\Delta t} d V+\int_{V} P_{m} \frac{\partial \rho u}{\partial x} d V+\int_{V} P_{m} \frac{\partial \rho w}{\partial z} d V=0, \text { para } m=1, \ldots, M
$$

onde $\rho^{t-\Delta t}$ é a massa específica calculada no instante de tempo anterior, $t-\Delta t$. Observa-se que não é utilizado nenhum símbolo para denotar as variáveis no instante $t$. A função peso $P_{m}$ segue a formulação de Petrov-Galerkin sendo dada, segundo Hughes e Brooks (1982), por:

$$
P_{m}^{i, j}=N_{\mathrm{m}}+\frac{\Delta t}{2} \bar{u}_{\mathrm{i}, \mathrm{j}} \frac{\partial N_{m}}{\partial x}+\frac{\Delta t}{2} \bar{w}_{\mathrm{i}, \mathrm{j}} \frac{\partial N_{m}}{\partial z}
$$

onde $\bar{u}_{i, j}$ é a velocidade média no elemento $i, j$, na direção $x$, e $\bar{w}_{i, j}$ é a velocidade média no elemento $i, j$ na direção $z$.

De forma análoga, pesando-se e integrando-se as equações de quantidade de movimento (4-2) e (4-3) para as direções $x$ e $z$, respectivamente, no elemento $i, j$ de volume $V$, obtém-se, respectivamente, o seguinte conjunto de equações para $u$ e $w$ no elemento $i, j$ :

$$
\begin{aligned}
& \int_{V} P_{m} \frac{\partial G_{x}}{\partial t} d V+\int_{V} P_{m} \frac{\partial G_{x} u}{\partial x} d V+\int_{V} P_{m} \frac{\partial G_{x} w}{\partial z} d V= \\
& =-\int_{V} P_{m} \frac{\partial p}{\partial x} d V+\int_{V} P_{m} \frac{4}{3} \mu \frac{\partial^{2} u}{\partial x^{2}} d V+\int_{V} P_{m} \mu \frac{\partial^{2} u}{\partial z^{2}} d V
\end{aligned}
$$

e

$$
\begin{gathered}
\int_{V} P_{m} \frac{\partial G_{z}}{\partial t} d V+\int_{V} P_{m} \frac{\partial G_{z} u}{\partial x} d V+\int_{V} P_{m} \frac{\partial G_{z} w}{\partial z} d V= \\
=-\int_{V} P_{m} \frac{\partial p}{\partial z} d V+\int_{V} P_{m} \mu \frac{\partial^{2} w}{\partial x^{2}} d V+\int_{V} P_{m} \frac{4}{3} \mu \frac{\partial^{2} w}{\partial z^{2}} d V
\end{gathered},
$$


Nas equações da quantidade de movimento, equações (4-17) e (4-18), aproxima-se também a derivada temporal, por uma diferença para trás, obtendo-se, respectivamente, as seguintes expressões para as velocidades nas direções $x$ e $z$,

$$
\begin{aligned}
& \int_{V} P_{m} \frac{G_{x}-G_{x}^{t-\Delta t}}{\Delta t} d V+\int_{V} P_{m} \frac{\partial G_{x} u}{\partial x} d V+\int_{V} P_{m} \frac{\partial G_{x} w}{\partial z} d V= \\
& =-\int_{V} P_{m} \frac{\partial p}{\partial x} d V+\int_{V} P_{m} \frac{4}{3} \mu \frac{\partial^{2} u}{\partial x^{2}} d V+\int_{V} P_{m} \mu \frac{\partial^{2} u}{\partial w^{2}} d V \quad, \text { para } m=1, \ldots, M,(4-19) \\
& \int_{V} P_{m} \frac{G_{z}-G_{z}^{t-\Delta t}}{\Delta t} d V+\int_{V} P_{m} \frac{\partial G_{z} u}{\partial x} d V+\int_{V} P_{m} \frac{\partial G_{z} w}{\partial z} d V= \\
& =-\int_{V} P_{m} \frac{\partial p}{\partial z} d V+\int_{V} P_{m} \mu \frac{\partial^{2} w}{\partial x^{2}} d V+\int_{V} P_{m} \frac{4}{3} \mu \frac{\partial^{2} w}{\partial z^{2}} d V \quad \text { para } m=1, \ldots, M, \quad(4-20)
\end{aligned}
$$

onde $G_{x}^{t-\Delta t}$ e $G_{z}^{t-\Delta t}$ são as velocidades mássicas, respectivamente, nas direções $x$ e $z$, calculadas no instante de tempo anterior. Novamente não é utilizado nenhum símbolo para denotar as variáveis no instante $t$.

Pesando-se e integrando-se as equações da velocidade mássica, nas direções $x$ e $z$, equações (4-4) e (4-5), respectivamente, no elemento $i, j$ de volume $V$, tem-se o seguinte:

$$
\int_{V} P_{m} G_{x} d V-\int_{V} P_{m} \rho u d V=0, \text { para } m=1, \ldots, M
$$

e

$$
\int_{V} P_{m} G_{z} d V-\int_{V} P_{m} \rho w d V=0, \text { para } m=1, \ldots, M
$$

Integrando-se e pesando-se a equação de estado, equação (4-6), no elemento $i, j$ de volume $V$, obtém-se a seguinte expressão: 


$$
\int_{V} P_{m} p d V-\int_{V} P_{m} \rho R T d V=0 \text {, para } m=1, \ldots, M .
$$

Integrando-se e pesando-se a equação de conservação da energia, dada pela equação (4-7), no elemento $i, j$ de volume $V$, obtém-se a seguinte expressão:

$$
\begin{aligned}
& \int_{V} P_{m} \frac{\partial E}{\partial t} d V+\int_{V} P_{m} \frac{\partial E u}{\partial x} d V+\int_{V} P_{m} \frac{\partial E w}{\partial z} d V= \\
& =-\int_{V} P_{m} p\left(\frac{\partial u}{\partial x}+\frac{\partial w}{\partial z}\right) d V+\int_{V} P_{m} K \nabla^{2} T d V+ \\
& +\int_{V} P_{m} 2 \mu\left(\left(\frac{\partial u}{\partial x}\right)^{2}+\frac{\partial u}{\partial x} \frac{\partial w}{\partial z}+\left(\frac{\partial w}{\partial z}\right)^{2}\right) d V-\int_{V} P_{m} \frac{2}{3} \mu\left[\left(\frac{\partial u}{\partial x}\right)+\left(\frac{\partial w}{\partial z}\right)\right] d V,
\end{aligned}
$$

para $m=1, \ldots, M$.

Analogamente às equações anteriores, aproxima-se a derivada temporal, presente na equação (4-24), por uma diferença para trás e obtém-se a seguinte equação:

$$
\begin{aligned}
& \int_{V} P_{m} \frac{E-E^{t-\Delta t}}{\Delta t} d V+\int_{V} P_{m} \frac{\partial E u}{\partial x} d V+\int_{V} P_{m} \frac{\partial E w}{\partial z} d V= \\
& =-\int_{V} P_{m} p\left(\frac{\partial u}{\partial x}+\frac{\partial w}{\partial z}\right) d V+\int_{V} P_{m} K \nabla^{2} T d V+ \\
& +\int_{V} P_{m} 2 \mu\left(\left(\frac{\partial u}{\partial x}\right)^{2}+\frac{\partial u}{\partial x} \frac{\partial w}{\partial z}+\left(\frac{\partial w}{\partial z}\right)^{2}\right) d V-\int_{V} P_{m} \frac{2}{3} \mu\left[\left(\frac{\partial u}{\partial x}\right)+\left(\frac{\partial w}{\partial z}\right)\right] d V,
\end{aligned}
$$

para $m=1, \ldots, M$,

onde $E^{t-\Delta t}$ é a energia calculada no instante de tempo anterior $t-\Delta t$.

Finalmente, integrando-se e pesando-se a equação da temperatura, dada pela equação (4-8), no elemento $i, j$ de volume $V$, obtém-se a seguinte equação: 


$$
\int_{V} P_{m} E d V-\int_{V} P_{m} \rho c v_{v} T d V=0, \text { para } m=1, \ldots, M
$$

Observa-se, novamente, que cada uma das equações (4-15), (4-19), (4-20), (4-21), (4-22), (4-23), (4-25) e (4-26) representam um sistema de $M$ equações para o elemento $i, j$.

A partir dessas equações, obtidas pela integração das equações (4-1) à (4-8) nos elementos da malha, aplica-se o método de expansão em funções hierárquicas para a obtenção das variáveis desejadas.

\subsection{1 - Equação da continuidade}

Para obtenção da expressão utilizada no cálculo da massa específica $(\rho)$, abrem-se as integrais da equação (4-15), para as direções $x$ e $z$, chegando-se ao seguinte:

$$
\iint_{x} P_{m} \frac{\rho-\rho^{t-\Delta t}}{\Delta t} d x d z+\iint_{x} P_{m} \frac{\partial \rho u}{\partial x} d x d z+\iint_{x z} P_{m} \frac{\partial \rho w}{\partial z} d x d z=0,
$$

para $m=1, \ldots, M$.

Nota-se que na equação (4-27) assume-se a largura unitária para o domínio na direção $y$.

A fim de facilitar o cálculo das integrais e, futuramente, o uso de geometrias irregulares faz-se a mudança do sistema de coordenadas $x$ e $z$, para $\xi$ e $\eta$ por meio das equações (4-9), (4-10), (4-11) e (4-12). Além disso, introduzindo-se a expansão das variáveis $\rho, G_{x}$ e $G_{z}$, dados por (4-13), obtém-se a seguinte expressão: 


$$
\begin{aligned}
& \sum_{n=1}^{M} \rho_{n}^{i, j}\left\{\frac{\Delta x_{i, j} \Delta z_{i, j}}{4 \Delta t} \int_{-1}^{1} \int_{-1}^{1} P_{m} N_{n} \mathrm{~d} \xi \mathrm{d} \eta+\frac{\Delta z_{i, j}}{2} \sum_{k=1}^{M} u_{\mathrm{k}}^{\mathrm{i}, \mathrm{j}} \int_{-1}^{1} \int_{-1}^{1} P_{m} \frac{\partial N_{n} N_{k}}{\partial \xi} \mathrm{d} \xi \mathrm{d} \eta+\right. \\
& \left.+\frac{\Delta x_{i, j}}{2} \sum_{k=1}^{M} w_{k}^{i, j} \int_{-1}^{1} \int_{-1}^{1} P_{m} \frac{\partial N_{\mathrm{n}} N_{\mathrm{k}}}{\partial \eta} \mathrm{d} \xi \mathrm{d} \eta\right\}=\frac{\Delta x_{i, j} \Delta z_{i, j}}{4 \Delta t} \sum_{n=1}^{M} \rho_{n}^{t-\Delta t} \int_{-1}^{1} \int_{-1}^{1} P_{m} N_{\mathrm{n}} \mathrm{d} \xi \mathrm{d} \eta \\
& \text { para } m=1, \ldots, M,
\end{aligned}
$$

onde os índices $i, j$, nas funções de expansão e peso, foram suprimidos para não sobrecarregar a notação.

Dividindo a equação (4-28) por $\frac{\Delta x_{i, j} \Delta z_{i, j}}{4}$ e colocando-a na forma matricial tem-se:

$$
\underline{A}^{i, j} \vec{\rho}^{i, j}=\vec{b}^{i, j}
$$

onde a matriz $\underline{A}^{i, j}$ é a matriz de expansão da variável $\rho$ no elemento $i, j, \vec{\rho}^{i, j}$ é o vetor dos coeficientes da massa específica $\rho$ no elemento $i, j$ e $\bar{b}^{i, j}$ é o vetor resultante para a massa específica no elemento i, j. Nota-se que a dimensão da matriz $\underline{A}^{i, j}$ é $(M \times M)$ e a dimensão dos vetores $\vec{\rho}^{i, j}$ e $\vec{b}^{i, j}$ é $(M \times 1)$. O elemento $(m, n)$ da matriz $\underline{A}^{i, j}$ é dado por:

$$
A_{m, n}^{i, j}=\frac{1}{\Delta t} \alpha_{m, n}^{i, j}+\frac{2}{\Delta x_{i, j}} \sum_{k=1}^{M} u_{k} \beta_{m, n, k}^{i, j}+\frac{2}{\Delta z_{i, j}} \sum_{k=1}^{M} w_{k} \gamma_{m, n, k}^{i, j},
$$

e o $m$-ésimo componente do vetor resultante, para o elemento $i, j$, é dado por:

$$
b_{\mathrm{m}}^{i, j}=\frac{1}{\Delta t} \sum_{m=1}^{M} \rho_{m}^{t-\Delta t} \alpha_{m, n}^{i, j} .
$$


Os parâmetros $\underline{\alpha}_{m, n}^{i, j}, \underline{\beta}_{m, n, k}^{i, j}$ e $\underline{\gamma}_{m, n, k}^{i, j}$ são definidos por integrais das funções peso e expansão, da seguinte forma:

$$
\begin{aligned}
& \alpha_{m, n}^{i, j}=\int_{-1}^{1} \int_{-1}^{1} P_{m} N_{n} d \xi d \eta=\int_{-1}^{1} \int_{-1}^{1} N_{m} N_{n}+\bar{u}^{i, j} d \xi d \eta \frac{\Delta t}{\Delta x_{i, j}} \int_{-1}^{1} \int_{-1}^{1} \frac{\partial N_{m}}{\partial \xi} N_{n} d \xi d \eta+ \\
& +\bar{w}^{i, j} \frac{\Delta t}{\Delta z_{i, j}} \int_{-1}^{1} \int_{-1}^{1} \frac{\partial N_{m}}{\partial \eta} N_{n} d \xi d \eta \\
& \beta_{m, n, k}^{i, j}=\int_{-1}^{1} \int_{-1}^{1} P_{m} \frac{\partial N_{n} N_{k}}{\partial \xi} d \xi d \eta=\int_{-1}^{1} \int_{-1}^{1} N_{m} \frac{\partial N_{n} N_{k}}{\partial \xi} d \xi d \eta+ \\
& +\bar{u}^{i, j} \frac{\Delta t}{\Delta x_{i, j}} \int_{-1-1}^{1} \int_{-1}^{1} \frac{\partial N_{m}}{\partial \xi} \frac{\partial N_{n} N_{k}}{\partial \xi} d \xi d \eta+\bar{w}^{i, j} \frac{\Delta t}{\Delta z_{i, j}} \int_{-1-1}^{1} \int_{-1}^{1} \frac{\partial N_{m}}{\partial \eta} \frac{\partial N_{n} N_{k}}{\partial \xi} d \xi d \eta \\
& \gamma_{m, n, k}^{i, j}=\int_{-1}^{1} \int_{-1}^{1} P_{m} \frac{\partial N_{n} N_{k}}{\partial \eta} d \xi d \eta=\int_{-1}^{1} \int_{-1}^{1} N_{m} \frac{\partial N_{n} N_{k}}{\partial \eta} d \xi d \eta+ \\
& +\bar{u}^{i, j} \frac{\Delta t}{\Delta x_{i, j}} \int_{-1-1}^{1} \int_{-1}^{1} \frac{\partial N_{m}}{\partial \xi} \frac{\partial N_{n} N_{k}}{\partial \eta} d \xi d \eta+\bar{w}^{i, j} \frac{\Delta t}{\Delta z_{i, j}} \int_{-1-1}^{1} \int_{-1}^{1} \frac{\partial N_{m}}{\partial \eta} \frac{\partial N_{n} N_{k}}{\partial \eta} d \xi d \eta .
\end{aligned}
$$

Observa-se que $P_{m}$ é a função peso dada pela equação (4-16). Nota-se que os parâmetros $\underline{\alpha}_{m, n}^{i, j}$ estão associados com o termo temporal e os parâmetros $\underline{\beta}_{m, n, k}^{i, j}$ e $\underline{\gamma}_{-m, n, k}^{i, j}$, com os termos convectivos. 


\subsection{2 - Equação da quantidade de movimento na direção $x$}

Para o cálculo da componente $G_{x}$ da velocidade mássica, aplica-se inicialmente o Teorema de Green na equação (4-19), obtendo-se o seguinte:

$$
\begin{aligned}
& \int_{V} P_{m} \frac{G_{x}-G_{x}^{t-\Delta t}}{\Delta t} d V+\int_{V} P_{m} \frac{\partial G_{x} u}{\partial x} d V+\int_{V} P_{m} \frac{\partial G_{x} w}{\partial z} d V=-\int_{V} P_{m} \frac{\partial p}{\partial x} d V+ \\
& +\frac{4}{3} \mu\left[\oint_{S} P_{m} \frac{\partial u}{\partial x} d S-\int_{V} \frac{\partial P_{m}}{\partial x} \frac{\partial u}{\partial x} d V\right]+\mu\left[\oint_{S} P_{m} \frac{\partial u}{\partial z} d S-\int_{V} \frac{\partial P_{m}}{\partial z} \frac{\partial u}{\partial z} d V\right], \\
& \operatorname{para} m=1, \ldots, M,
\end{aligned}
$$

onde $S$ é a área externa do domínio da solução. Observa-se que os termos que envolvem integrais de superfície somente aparecem para os elementos localizados nas regiões de contorno da malha.

Abrindo as integrais da equação anterior, para as direções $x$ e $z$, tem-se a seguinte equação:

$$
\begin{aligned}
& \iint_{x} P_{m} \frac{G_{x}-G_{x}^{t-\Delta t}}{\Delta t} d x d z+\iint_{x z} P_{m} \frac{\partial G_{x} u}{\partial x} d x d z+\iint_{x z} P_{m} \frac{\partial G_{x} w}{\partial z} d x d z= \\
& =-\iint_{x z} P_{m} \frac{\partial p}{\partial x} d x d z+\frac{4}{3} \mu\left[\iint_{z}\left(P_{m} \frac{\partial u}{\partial x}\right)_{x=L x} d z-\iint_{x} \frac{\partial P_{m}}{\partial x} \frac{\partial u}{\partial x} d x d z\right]+ \\
& +\mu\left[\int_{x}\left(P_{m} \frac{\partial u}{\partial z}\right)_{z=L z} d x-\int_{x} \int_{z} \frac{\partial P_{m}}{\partial z} \frac{\partial u}{\partial z} d x d z\right], \\
& \operatorname{para} m=1, \ldots, M,
\end{aligned}
$$

onde $L x$ e $L z$ representam simbolicamente os respectivos extremos $x$ e $z$ do domínio da solução. 
Fazendo-se a mudança do sistema de coordenadas $x$ e $z$ para as variáveis locais $\xi$ e $\eta$, de acordo, respectivamente, com as equações (4-9), (4-10), (4-11) e (4-12) e introduzindo-se a expansão para as variáveis $\rho, u, w, G_{x}$, e $p$ dadas por (4-13), obtém-se a seguinte equação:

$$
\begin{aligned}
& \sum_{n=1}^{M} G_{x}\left\{\frac{\Delta x_{i, j} \Delta z_{i, j}}{4 \Delta t} \int_{-1}^{1} \int_{-1}^{1} P_{m} N_{n} d \xi d \eta+\frac{\Delta z_{i, j}}{2} \sum_{k=1}^{M} u_{k} \int_{-1}^{1} \int_{-1}^{1} P_{m} \frac{\partial N_{n} N_{k}}{\partial \xi} d \xi d \eta+\right. \\
& \left.+\frac{\Delta x_{i, j}}{2} \sum_{k=1}^{M} w_{k} \int_{-1}^{1} \int_{-1}^{1} P_{m} \frac{\partial N_{n} N_{k}}{\partial \eta} d \xi d \eta\right\}=\frac{\Delta x_{i, j} \Delta z_{i, j}}{4 \Delta T} \sum_{n=1}^{M} G_{x n}^{t-\Delta t} \int_{-1}^{1} \int_{-1}^{1} P_{m} N_{n} d \xi d \eta- \\
& -\frac{\Delta z_{i, j}}{2} \sum_{n=1}^{M} p_{n} \int_{-1}^{1} \int_{-1}^{1} P_{m} \frac{\partial N_{n}}{\partial \xi} d \xi d \eta+\sum_{n=1}^{M} u_{n}\left\{\frac { 4 } { 3 } \mu \frac { \Delta z _ { i , j } } { \Delta x _ { i , j } } \left[\int_{-1}^{1}\left(P_{m} \frac{\partial N_{n}}{\partial \xi}\right)_{\xi=-1}^{\xi=1} d \eta-\right.\right. \\
& \left.\left.-\int_{-1}^{1} \int_{-1}^{1} \frac{\partial P_{m}}{\partial \xi} \frac{\partial N_{n}}{\partial \xi} d \xi d \eta\right]+\mu \frac{\Delta x_{i, j}}{\Delta z_{i, j}}\left[\int_{-1}^{1}\left(P_{m} \frac{\partial N_{n}}{\partial \eta}\right)_{\eta=-1}^{\eta=1} d \xi-\int_{-1}^{1} \int_{-1}^{1} \frac{\partial P_{m}}{\partial \eta} \frac{\partial N_{n}}{\partial \eta} d \xi d \eta\right]\right\} \\
& \operatorname{para} m=1, \ldots, M .
\end{aligned}
$$

Observa-se novamente que os termos de integral de superfície somente aparecem nos elementos localizados nas regiões de contorno do domínio da solução. Dividindo a equação (4-37) por $\frac{\Delta x_{i, j} \Delta z_{i, j}}{4}$ e colocando-a na forma matricial tem-se:

$$
\underline{A}^{i, j} \vec{G}_{x}^{i, j}=\vec{b}_{x}^{i, j}
$$

onde a matriz $\underline{A}^{i, j}$ é a matriz de expansão dada pela equação (4-30), $\vec{G}_{x}^{i, j}$ é o vetor dos coeficientes da velocidade mássica $G_{x}$ para o elemento $i, j$ e $\vec{b}_{x}^{i, j}$ é o vetor resultante para a velocidade mássica para o $m$-ésimo componente do vetor resultante, para o elemento $i, j$, que é dado por: 


$$
\begin{aligned}
b_{x}^{i, j}= & \frac{1}{\Delta t} \sum_{n=1}^{M} G_{x n}^{t-\Delta t} \alpha_{m, n}^{i, j}-\frac{2}{\Delta x_{i, j}} \sum_{n=1}^{K} p_{n} \Omega_{m, n}^{i, j}+ \\
& +\sum_{n=1}^{M} u_{n}\left[\frac{16 \mu}{3 \Delta x_{i, j}^{2}}\left(C_{z m, n}^{i, j}-D_{x m, n}^{i, j}\right)+\frac{4 \mu}{\Delta z_{i, j}^{2}}\left(C_{x m, n}^{i, j}-D_{z m, n}^{i, j}\right)\right]
\end{aligned}
$$

Os parâmetros $\underline{C}_{x}^{i, j}, \underline{C}_{z}^{i, j}, \underline{D}_{x}^{i, j}, \underline{D}_{z}^{i, j}$ e $\underline{\Omega}^{i, j}$ são definidos por integrais das funções peso e expansão, da seguinte forma:

$$
\begin{aligned}
C_{z m, n}^{i, j} & =\int_{-1}^{1}\left(P_{m} \frac{\partial N_{n}}{\partial \xi}\right)_{\xi=-1}^{\xi=1} d \eta=\left.f c d \int_{-1}^{1} P_{m} \frac{\partial N_{n}}{\partial \xi}\right|_{\xi=1} d \eta-\left.f c e \int_{-1}^{1} P_{m} \frac{\partial N_{n}}{\partial \xi}\right|_{\xi=-1} d \eta= \\
& =f c d\left[\int_{-1}^{1} N_{m} \frac{\partial N_{n}}{\partial \xi} d \eta+\bar{u}^{i, j} \frac{\Delta t}{\Delta x_{i, j}} \int_{-1}^{1} \frac{\partial N_{m}}{\partial \xi} \frac{\partial N_{n}}{\partial \xi} d \eta+\bar{w}^{i, j} \frac{\Delta t}{\Delta z i, j} \int_{-1}^{1} \frac{\partial N_{m}}{\partial \eta} \frac{\partial N_{n}}{\partial \xi} d \eta\right]_{\xi=1}- \\
& -f c e\left[\int_{-1}^{1} N_{m} \frac{\partial N_{n}}{\partial \xi} d \eta+\bar{u}^{i, j} \frac{\Delta t}{\Delta x_{i, j}} \int_{-1}^{1} \frac{\partial N_{m}}{\partial \xi} \frac{\partial N_{n}}{\partial \xi} d \eta+\bar{w}^{i, j} \frac{\Delta t}{\Delta z i, j} \int_{-1}^{1} \frac{\partial N_{m}}{\partial \eta} \frac{\partial N_{n}}{\partial \xi} d \eta\right]_{\xi=-1} \\
C_{x m, n}^{i, j} & =\int_{-1}^{1}\left(P_{m} \frac{\partial N_{n}}{\partial \eta}\right)_{\eta=-1}^{\eta=1} d \xi=\left.f c s \int_{-1}^{1} P_{m} \frac{\partial N_{n}}{\partial \eta}\right|_{\eta=1} d \xi-\left.f c i \int_{-1}^{1} P_{m} \frac{\partial N_{n}}{\partial \eta}\right|_{\eta=-1} ^{d \xi}= \\
= & f c s\left[\int_{-1}^{1} N_{m} \frac{\partial N_{n}}{\partial \eta} d \xi+\bar{u}^{i, j} \frac{\Delta t}{\Delta x_{i, j}} \int_{-1}^{1} \frac{\partial N_{m}}{\partial \xi} \frac{\partial N_{n}}{\partial \eta} d \xi+\bar{w}^{i, j} \frac{\Delta t}{\Delta \int_{i, j}-1} \frac{\partial N_{m}}{\partial \eta} \frac{\partial N_{n}}{\partial \eta} d \xi\right]_{\eta=1}
\end{aligned}
$$




$$
\begin{aligned}
& -f c i\left[\int_{-1}^{1} N_{m} \frac{\partial N_{n}}{\partial \eta} d \xi+\bar{u}^{i, j} \frac{\Delta t}{\Delta x_{i, j}} \int_{-1}^{1} \frac{\partial N_{m}}{\partial \xi} \frac{\partial N_{n}}{\partial \eta} d \xi+\bar{w}^{i, j} \frac{\Delta t}{\Delta z_{i, j}} \int_{-1}^{1} \frac{\partial N_{m}}{\partial \eta} \frac{\partial N_{n}}{\partial \eta} d \xi\right]_{\eta=-1} \\
& \begin{aligned}
D_{x m, n}^{i, j}= & \int_{-1-1}^{1} \int_{-1}^{1} \frac{\partial P_{m}}{\partial \xi} \frac{\partial N_{n}}{\partial \xi} d \xi d \eta=\int_{-1}^{1} \int_{-1}^{1} \frac{\partial N_{m}}{\partial \xi} \frac{\partial N_{n}}{\partial \xi} d \xi d \eta+ \\
& +\bar{u}^{i, j} \frac{\Delta t}{\Delta x_{i, j}} \int_{-1-1}^{1} \int_{-1}^{1} \frac{\partial^{2} N_{n}}{\partial \xi^{2}} \frac{\partial N_{n}}{\partial \xi} d \xi d \eta+\bar{w}^{i, j} \frac{\Delta t}{\Delta z_{i, j}} \int_{-1-1}^{1} \int_{-1}^{1} \frac{\partial^{2} N_{m}}{\partial \eta \partial \xi} \frac{\partial N_{n}}{\partial \xi} d \xi d \eta
\end{aligned} \\
& D_{z m, n}^{i, j}=\int_{-1-1}^{1} \int_{-1}^{1} \frac{\partial P_{m}}{\partial \eta} \frac{\partial N_{n}}{\partial \eta} d \xi d \eta=\int_{-1}^{1} \int_{-1}^{1} \frac{\partial N_{m}}{\partial \eta} \frac{\partial N_{n}}{\partial \eta} d \xi d \eta+ \\
& +\bar{u}^{i, j} \frac{\Delta t}{\Delta x_{i, j}} \int_{-1-1}^{1} \int_{-1}^{1} \frac{\partial^{2} N_{n}}{\partial \eta \partial \xi} \frac{\partial N_{n}}{\partial \eta} d \xi d \eta+\bar{w}^{i, j} \frac{\Delta t}{\Delta z i, j} \int_{-1-1}^{1} \int_{-1}^{1} \frac{\partial^{2} N_{m}}{\partial^{2} \eta} \frac{\partial N_{n}}{\partial \eta} d \xi d \eta \\
& \Omega_{m, n}^{i, j}=\int_{-1}^{1} \int_{-1}^{1} P_{m} \frac{\partial N_{n}}{\partial \xi} d \xi d \eta=\int_{-1}^{1} \int_{-1}^{1} N_{m} \frac{\partial N_{n}}{\partial \xi} d \xi d \eta+ \\
& +\bar{u}^{i, j} \frac{\Delta t}{\Delta x_{i, j}} \int_{-1-1}^{1} \int_{-1}^{1} \frac{\partial N_{m}}{\partial \xi} \frac{\partial N_{n}}{\partial \xi} d \xi d \eta+\bar{w}^{i, j} \frac{\Delta t}{\Delta z_{i, j}} \int_{-1-1}^{1} \int_{-1}^{1} \frac{\partial N_{m}}{\partial \eta} \frac{\partial N_{n}}{\partial \xi} d \xi d \eta .
\end{aligned}
$$

Observa-se que os parâmetros $\alpha_{m, n}^{i, j}$ estão associados com o termo temporal; os parâmetros $\Omega_{m, n}^{i, j}$, com os termos convectivos; os parâmetros $D_{x m, n}^{i, j}$ e $D_{z m, n}^{i, j}$, com os termos difusivos; os parâmetros $C_{x m, n}^{i, j}$, com as condições de contorno nas faces $x$ do elemento; e a matriz $C_{z m, n}^{i, j}$, com as condições de contorno nas faces $z$ do elemento. 
Os termos $f e d, f c e, f c s$ e $f c i$, que aparecem nos parâmetros de contorno $C_{x}^{i, j}{ }_{m, n}$ e $C_{z}^{i} j_{m, n}$, são denominados fatores de contorno e são definidos como se segue:

fed: é igual a 1 , se a face direita do elemento $i, j$ estiver no contorno do domínio da solução e igual a zero, se não estiver;

$f c e$ : é igual a 1 , se a face esquerda do elemento $i$, j estiver no contorno do domínio da solução e igual a zero, se não estiver;

$f C s$ : é igual a 1 , se a face superior do elemento $i, j$ estiver no contorno do domínio da solução e igual a zero, se não estiver;

$f c i$ : é igual a 1 , se a face inferior do elemento $i, j$ estiver no contorno do domínio da solução e igual a zero, se não estiver;

\subsection{3 - Equação da quantidade de movimento na direção $\mathrm{z}$}

Para o cálculo da componente $w$ da velocidade mássica, aplica-se, inicialmente, o Teorema de Green na equação (4-20), obtendo-se a seguinte equação:

$$
\begin{aligned}
\int_{V} P_{m} \frac{G_{z}-G_{z}^{t-\Delta t}}{\Delta t} d V+\int_{V} P_{m} \frac{\partial G_{z} u}{\partial x} d V+\int_{V} P_{m} \frac{\partial G_{z} w}{\partial z} d V=-\int_{V} P_{m} \frac{\partial p}{\partial z} d V+ \\
+\frac{4}{3} \mu\left[\oint_{S} P_{m} \frac{\partial w}{\partial z} d S-\int_{V} \frac{\partial P_{m}}{\partial z} \frac{\partial w}{\partial z} d V\right]+\mu\left[\oint_{S} P_{m} \frac{\partial w}{\partial x} d S-\int_{V} \frac{\partial P_{m}}{\partial x} \frac{\partial w}{\partial x} d V\right]
\end{aligned}
$$

para $m=1, \ldots, M$,

onde $S$ é a área externa do elemento $i, j$. 
Abrindo as integrais da equação anterior, para as direções $x$ e $z$, tem-se a seguinte equação:

$$
\begin{gathered}
\iint_{x z} P_{m} \frac{G_{z}-G_{z}^{t-\Delta t}}{\Delta t} d x d z+\iint_{x} P_{m} \frac{\partial G_{z} u}{\partial x} d x d z+\iint_{x} P_{m} \frac{\partial G_{z} w}{\partial z} d x d z= \\
=-\iint_{x} P_{m} \frac{\partial p}{\partial z} d x d z+\frac{4}{3} \mu\left[\iint_{x}\left(P_{m} \frac{\partial w}{\partial z}\right)_{z=L z} d x-\iint_{x z} \frac{\partial P_{m}}{\partial z} \frac{\partial w}{\partial z} d x d z\right]+ \\
+\mu\left[\int_{z}\left(P_{m} \frac{\partial w}{\partial x}\right)_{x=L x} d z-\int_{x z} \frac{\partial P_{m}}{\partial x} \frac{\partial w}{\partial x} d x d z\right], \text { para } m=1, \ldots, M,
\end{gathered}
$$

Fazendo-se a mudança do sistema de coordenadas $x$ e $z$ para as variáveis locais $\xi$ e $\eta$, de acordo, respectivamente, com as equações (4-9), (4-10), (4-11) e (4-12) e introduzindo-se a expansão para as variáveis $\rho, u, w, G_{z}$ e $p$ dadas por (4-13), obtém-se a seguinte equação:

$$
\begin{aligned}
& \sum_{n=1}^{M} G_{z n}\left\{\frac{\Delta x_{i, j} \Delta z_{i, j}}{4 \Delta t} \int_{-1}^{1} \int_{-1}^{1} P_{m} N_{n} d \xi d \eta+\frac{\Delta z_{i, j}}{2} \sum_{k=1}^{M} u_{k} \int_{-1}^{1} \int_{-1}^{1} P_{m} \frac{\partial N_{n} N_{k}}{\partial \xi} d \xi d \eta+\right. \\
& \left.+\frac{\Delta x_{i, j}}{2} \sum_{k=1}^{M} w_{k} \int_{-1}^{1} \int_{-1}^{1} P_{m} \frac{\partial N_{n} N_{k}}{\partial \eta} d \xi d \eta\right\}=\frac{\Delta x_{i, j} \Delta z_{i, j}}{4 \Delta T} \sum_{n=1}^{M} G_{z}^{t-\Delta t} \int_{-1}^{1} \int_{-1}^{1} \rho_{m} N_{n} d \xi d \eta- \\
& -\frac{\Delta x_{i, j}}{2} \sum_{n=1}^{M} p_{n} \int_{-1}^{1} \int_{-1}^{1} P_{m} \frac{\partial N_{n}}{\partial \eta} d \xi d \eta+\sum_{n=1}^{M} w_{n}\left\{\frac { 4 } { 3 } \mu \frac { \Delta x _ { i , j } } { \Delta z _ { i , j } } \left[\int_{-1}^{1}\left(P_{m} \frac{\partial N_{n}}{\partial \eta}\right)_{\eta=-1}^{\eta=1} d \xi-\right.\right. \\
& \left.\left.-\int_{-1}^{1} \int_{-1}^{1} \frac{\partial P_{m}}{\partial \eta} \frac{\partial N_{n}}{\partial \eta} d \xi d \eta\right]+\mu \frac{\Delta z_{i, j}}{\Delta x_{i, j}}\left[\int_{-1}^{1}\left(P_{m} \frac{\partial N_{n}}{\partial \xi}\right)_{\xi=-1}^{\xi=1} d \eta-\int_{-1}^{1} \int_{-1}^{1} \frac{\partial P_{m}}{\partial \xi} \frac{\partial N_{n}}{\partial \xi} d \xi d \eta\right]\right\}, \\
& \operatorname{para} m=1, \ldots, M .
\end{aligned}
$$


Dividindo a equação (4-47) por $\frac{\Delta x_{i, j} \Delta z_{i, j}}{4}$ e colocando-a na forma matricial tem-se:

$$
\underline{A}^{i, j} \vec{G}_{z}^{i, j}=\vec{b}_{z}^{i, j}
$$

onde a matriz $\underline{A}^{i, j}$ é a matriz de expansão e é dada pela equação (4-30), $\vec{G}_{z}^{i, j}$ é o vetor dos coeficientes da velocidade mássica $G_{z}$ para o elemento $i, j$ e $\vec{b}_{z}^{i, j}$ é o vetor resultante para a velocidade mássica $G_{z}$ para o elemento $i, j$. O $m$-ésimo componente do vetor resultante, para o elemento $i, j$, é dado por:

$$
\begin{aligned}
b_{z}^{i, j}= & \frac{1}{\Delta t} \sum_{n=1}^{M} G_{z n}^{t-\Delta t} \alpha_{m, n}^{i, j}-\frac{2}{\Delta x_{i, j}} \sum_{n=1}^{K} p_{n} \psi_{m, n}^{i, j}+ \\
& +\sum_{n=1}^{M} \omega_{n}\left[\frac{16 \mu}{3 \Delta z_{i, j}^{2}}\left(C_{x m, n}^{i, j}-D_{z m, n}^{i, j}\right)+\frac{4 \mu}{\Delta x_{i, j}^{2}}\left(C_{z m, n}^{i, j}-D_{x m, n}^{i, j}\right)\right],
\end{aligned}
$$

os parâmetros $\alpha_{m, n}^{i, j}, \quad C_{x_{m, n}}^{i, j}, C_{z_{m, n}}^{i, j}, \quad D_{x_{m, n}}^{i, j}$ e $D_{z_{m, n}}^{i, j}$ são as integrais já definidas anteriormente e o parâmetro $\psi_{m, n}^{i, j}$ é definido por:

$$
\begin{aligned}
\psi_{m, n}^{i, j}= & \int_{-1}^{1} \int_{-1}^{1} P_{m} \frac{\partial N_{n}}{\partial \eta} d \xi d \eta=\int_{-1}^{1} \int_{-1}^{1} N_{m} \frac{\partial N_{n}}{\partial \eta} d \xi d \eta+\bar{u}^{i, j} \frac{\Delta t}{\Delta x_{i, j}} \int_{-1-1}^{1} \int_{-1}^{1} \frac{\partial N_{m}}{\partial \xi} \frac{\partial N_{n}}{\partial \eta} d \xi d \eta+ \\
& +\bar{w}^{i, j} \frac{\Delta t}{\Delta z_{i, j}} \int_{-1-1}^{1} \int_{-1}^{1} \frac{\partial N_{m}}{\partial \eta} \frac{\partial N_{n}}{\partial \eta} d \xi d \eta ;
\end{aligned}
$$




\subsection{4 - Equação da velocidade mássica na direção $\mathrm{x}$}

A partir da equação integrada e pesada da velocidade mássica na direção $x$, dada por (4-21), abrem-se as integrais nas respectivas direções $x$ e $z$, resultando no seguinte:

$$
\iint_{x} P_{m} G_{x} d x d z-\iint_{x} P_{m} \rho u d x d z=0, \text { para } m=1, \ldots, M
$$

Faz-se a mudança de variáveis de $x$ e $z$ para $\xi$ e $\eta$, e introduz-se a expansão para as variáveis $G_{x}, \rho$ e $u$, dadas por (4-13), obtendo-se a seguinte equação:

$$
\sum_{n=1}^{M} u_{n} \sum_{k=1}^{M} \rho_{k} \int_{-1}^{1} \int_{-1}^{1} P_{m} N_{n} N_{k} d \xi d \eta=\sum_{n=1}^{M} G_{x \mathrm{n}} \int_{-1}^{1} \int_{-1}^{1} P_{m} N_{n} d \xi d \eta, \text { para } m=1, \ldots, M
$$

Escrevendo a equação anterior na forma matricial, para o cálculo da velocidade $u$, tem-se:

$$
\underline{A}_{u}^{i, j} \vec{u}^{i, j}=\vec{b}_{u}^{i, j}
$$

onde a matriz $\underline{A}_{u}^{i, j}$ é a matriz de expansão da variável $u$ para o elemento $i, j, \vec{u}^{i, j}$ é o vetor dos coeficientes da componente de velocidade $u$ para o elemento $i, j$ e $\vec{b}_{u}^{i, j}$ é o vetor resultante para a velocidade $u$ no elemento $i, j$. Nota-se que a dimensão da matriz $\underline{A}_{u}^{i, j}$ é $(M \times M)$ e a dimensão dos vetores $\vec{u}^{i, j}$ e $\vec{b}_{u}^{i, j}$ é $(M \times 1)$. O elemento $(m, n)$ da matriz $\underline{A}_{u}^{i, j}$ é dado por:

$$
A_{u m, n}^{i, j}=\sum_{k=1}^{M} \rho_{k} \theta_{m, n, k}^{i, j}
$$


onde o parâmetro $\theta_{m, n, k}^{i, j}$ é definido por:

$$
\begin{gathered}
\theta_{m, n, k}^{i, j}=\int_{-1-1}^{1} \int_{m}^{1} P_{m} N_{n} N_{k} d \xi d \eta=\int_{-1-1}^{1} \int_{m}^{1} N_{m} N_{n} N_{k} d \xi d \eta+\bar{u}^{i, j} \frac{\Delta t}{\Delta x_{i, j}} \int_{-1-1}^{1} \int_{-1}^{1} \frac{\partial N_{m}}{\partial \xi} N_{n} N_{k} d \xi d \eta+ \\
\quad+\bar{w}^{i, j} \frac{\Delta t}{\Delta z} \int_{i, j}^{1} \int_{-1-1}^{1} \frac{\partial N_{m}}{\partial \eta} N_{n} N_{k} d \xi d \eta
\end{gathered}
$$

O $m$-ésimo componente do vetor resultante para o elemento $i, j$ é dado pela expressão:

$$
b_{u m, n}^{i, j}=\sum_{n=1}^{M} G_{x n} \alpha_{m, n}^{i, j},
$$

onde $\alpha_{m, n}^{i, j}$ é a integral já definida anteriormente.

\subsection{5 - Equação da velocidade mássica na direção z}

Na seqüência, desenvolve-se a expressão utilizada para o cálculo da velocidade na direção $z$, a partir da equação (4-22), cujas integrais são abertas nas respectivas direções $x$ e $z$, resultando no seguinte:

$$
\iint_{x} P_{m} G_{z} d x d z-\iint_{x} P_{m} \rho w d x d z=0, \text { para } m=1, \ldots, M
$$

Realiza-se a mudança de variáveis de $x$ e $z$ para $\xi$ e $\eta$ e introduz-se a expansão para as variáveis $G_{z}, \rho$ e $w$, dadas por (4-13), obtendo-se a seguinte equação: 


$$
\sum_{n=1}^{M} w_{n} \sum_{k=1}^{M} \rho_{k} \int_{-1}^{1} \int_{-1}^{1} P_{m} N_{n} N_{k} d \xi d \eta=\sum_{n=1}^{M} G_{z \mathrm{n}} \int_{-1}^{1} \int_{-1}^{1} P_{m} N_{n} d \xi d \eta, \text { para } m=1, \ldots, M
$$

Reescrevendo a equação (4-58) na forma matricial, tem-se uma expressão para o cálculo da velocidade $w$, ou seja:

$$
\underline{A}_{w}^{i, j} \vec{w}^{i, j}=\vec{b}_{w}^{i, j},
$$

onde a matriz $\underline{A}_{w}^{i, j}$ é idêntica à matriz de expansão da variável $u$, equação (4-54), $\vec{w}^{i, j}$ é o vetor dos coeficientes da componente de velocidade $w$ para o elemento $i, j$ e $\vec{b}_{w}^{i, j}$ é o vetor resultante para a velocidade $w$ para o elemento $i, j$. O elemento $(m, n)$ da matriz $\underline{A}_{w}^{i, j}$ é dado por:

$$
A_{w m, n}^{i, j}=\sum_{k=1}^{M} \rho_{k} \theta_{m, n, k}^{i, j}
$$

O $m$-ésimo componente do vetor resultante para o elemento $i$, $j$ é dado pela expressão:

$$
b_{w m, n}^{i, j}=\sum_{n=1}^{M} G_{z n} \alpha_{m, n}^{i, j} .
$$

Os parâmetros $\alpha_{m, n}^{i, j}$ e $\theta_{m, n, k}^{i, j}$ são as integrais já definidas anteriormente.

\subsection{6 - Equação de estado}

A partir da equação de estado integrada e pesada, dada por (4-23), abrem-se as integrais nas direções $x$ e $z$, respectivamente, obtendo-se a seguinte equação: 


$$
\iint_{x z} P_{m} p d x d z-\iint_{x z} P_{m} \rho R T d x d z=0 .
$$

O mesmo procedimento matemático adotado para as equações anteriores é utilizado nesse caso, ou seja, faz-se a mudança de variáveis de $x$ e $z$ para $\xi$ e $\eta$ e introduz-se a expansão para as variáveis $p, \rho$ e $T$, dadas por (4-13), obtendo-se a seguinte expressão:

$$
\begin{aligned}
& \sum_{n=1}^{M} p_{n} \int_{-1}^{1} \int_{-1}^{1} P_{m} N_{n} d \xi d \eta-R \sum_{n=1}^{M} \rho_{n} \sum_{k=1}^{M} T_{k} \int_{-1}^{1} \int_{-1}^{1} P_{m} N_{n} N_{k} d \xi d \eta=0 \\
& \text { para } m=1, \ldots, M .
\end{aligned}
$$

Reescrevendo a equação anterior na forma matricial tem-se:

$$
\underline{A}_{p}^{i, j} \vec{p}^{i, j}=\vec{b}_{p}^{i, j}
$$

onde a matriz $\underline{A}_{p}^{i, j}$ é a matriz de expansão da variável $p$ para o elemento $i, j, \vec{p}^{i, j}$ é o vetor dos coeficientes da pressão para o elemento $i, j$ e $\vec{b}_{p}^{i, j}$ é o vetor resultante para a pressão no elemento $i, j$. Nota-se que a dimensão da matriz $\underline{A}_{p}^{i, j}$ é $(M \times M)$ e a dimensão dos vetores $\vec{p}^{i, j}$ e $\vec{b}_{p}^{i, j}$ é $(M \mathrm{x} 1)$. O elemento $(m, n)$ da matriz $\underline{A}_{p}^{i, j}$ é dado por:

$$
A_{p m, n}^{i, j}=\alpha_{m, n}^{i, j}
$$

O $m$-ésimo componente do vetor resultante para o elemento $i$, $j$ é dado pela expressão:

$$
b_{p m, n}^{i, j}=R \sum_{n=1}^{M} \rho_{n} \sum_{k=1}^{M} T_{k} \theta_{m, n, k}^{i, j} .
$$


Os parâmetros $\alpha_{n, n}^{i, j}$ e $\theta_{m, n, k}^{i, j}$ são as integrais já definidas anteriormente.

\subsection{7 - Equação da conservação de energia}

Com base na equação integrada e pesada da energia, dada por (4-25), aplicando-se o teorema de Green para os termos difusivos, a mesma pode ser reescrita da seguinte forma:

$$
\begin{aligned}
& \frac{1}{\Delta t} \int_{V} P_{m} E d V+\int_{V} P_{m} \frac{\partial E u}{\partial x} d V+\int_{V} P_{m} \frac{\partial E w}{\partial z} d V=\frac{1}{\Delta t} \int_{V} P_{m} E^{t-\Delta t} d V- \\
& -\int_{V} P_{m} p\left(\frac{\partial u}{\partial x}+\frac{\partial w}{\partial z}\right) d V+K\left[\oint_{S} P_{m} \frac{\partial T}{\partial x} d S-\int_{V} \frac{\partial P_{m}}{\partial x} \frac{\partial T}{\partial x} d V\right]+ \\
& +K\left[\oint_{S} P_{m} \frac{\partial T}{\partial z} d S-\int_{V} \frac{\partial P_{m}}{\partial z} \frac{\partial T}{\partial z} d V\right]+2 \mu \int_{V} P_{m}\left(\left(\frac{\partial u}{\partial x}\right)^{2}+\frac{\partial u}{\partial x} \frac{\partial w}{\partial z}+\left(\frac{\partial w}{\partial z}\right)^{2}\right) d V- \\
& -\frac{2}{3} \mu \int_{V} P_{m}\left[\left(\frac{\partial u}{\partial x}\right)+\left(\frac{\partial w}{\partial z}\right)\right] d V \text {, para } m=1, \ldots, M .
\end{aligned}
$$

Abrindo-se as integrais nas direções $x$ e $z$, fazendo a mudança de variáveis de $x$ e $z$ para $\xi$ e $\eta$ e, finalmente, introduzindo a expansão para as variáveis $\rho, T, G_{x}$ e $G_{z}$, conforme (4-13), resulta na seguinte expressão: 


$$
\begin{aligned}
& \sum_{n=1}^{M} E_{n}\left\{\frac{\Delta x_{i, j} \Delta z_{i, j}}{4 \Delta t} \int_{-1}^{1} \int_{-1}^{1} P_{m} N_{n} d \xi d \eta+\sum_{k=1}^{M} u_{k} \frac{\Delta z_{i, j}}{2} \int_{-1}^{1} \int_{-1}^{1} P_{m} \frac{\partial N_{n} N_{k}}{\partial \xi} d \xi d \eta+\right. \\
& \left.+\sum_{k=1}^{M} w_{k} \frac{\Delta x_{i, j}}{2} \int_{-1}^{1} \int_{-1}^{1} P_{m} \frac{\partial N_{n} N_{k}}{\partial \eta} d \xi d \eta\right\}=\frac{\Delta x_{i, j} \Delta z_{i, j}}{4 \Delta t} \sum_{n=1}^{M} E_{n}^{t-\Delta t} \int_{-1}^{1} \int_{-1}^{1} P_{m} N_{n} d \xi d \eta+ \\
& +\sum_{n=1}^{M} T_{n}\left\{K \frac{\Delta z_{i, j}}{\Delta x_{i, j}}\left[\int_{-1}^{1}\left(P_{m} \frac{\partial N_{n}}{\partial \xi}\right)_{\xi=-1}^{\xi=1} d \eta-\int_{-1}^{1} \int_{-1}^{1} \frac{\partial N_{m}}{\partial \xi} \frac{\partial N_{n}}{\partial \xi} d \xi d \eta\right]+\right. \\
& \left.+K \frac{\Delta x_{i, j}}{\Delta z_{i, j}}\left[\int_{-1}^{1} P_{m}\left(\frac{\partial N_{n}}{\partial \eta}\right)_{\eta=-1}^{\eta=1} d \xi-\int_{-1}^{1} \int_{-1}^{1} \frac{\partial N_{m}}{\partial \eta} \frac{\partial T}{\partial \eta} d \xi d \eta\right]\right\}+ \\
& +2 \mu\left\{\frac{\Delta z_{i, j}}{\Delta x_{i, j}} \sum_{n=1}^{M} u_{n} \sum_{k=1}^{M} u_{k} \int_{-1}^{1} \int_{-1}^{1} P_{m} \frac{\partial N_{n}}{\partial \xi} \frac{\partial N_{k}}{\partial \xi} \mathrm{d} \xi \mathrm{d} \eta+\right. \\
& +\sum_{n=1}^{M} u_{n} \sum_{r r=1}^{K} w_{k} \int_{-1}^{1} \int_{-1}^{1} P_{m} \frac{\partial N_{n}}{\partial \xi} \frac{\partial N_{k}}{\partial \eta} d \xi d \eta+ \\
& \left.+\frac{\Delta x_{i, j}}{\Delta z_{i, j}} \sum_{n=1}^{M} w_{n} \sum_{k=1}^{M} w_{k} \int_{-1}^{1} \int_{-1}^{1} P_{m}+\frac{\partial N_{n}}{\partial \eta} \frac{\partial N_{k}}{\partial \eta} d \xi d \eta\right\}- \\
& -\frac{\mu}{3}\left[\Delta z_{i, j} \sum_{n=1}^{M} u_{n} \int_{-1}^{1} \int_{-1}^{1} P_{m} \frac{\partial N_{n}}{\partial \xi} d \xi d \eta+\Delta x_{i, j} \sum_{n=1}^{M} w_{n} \int_{-1}^{1} \int_{-1}^{1} P_{m} \frac{\partial N_{n}}{\partial \eta} d \xi d \eta\right]+ \\
& +\sum_{n=1}^{M} p_{n}\left\{\frac{2}{\Delta x} \sum_{k=1}^{M} u_{k} \int_{-1}^{1} \int_{-1}^{1} P_{m} N_{n} \frac{\partial N_{k}}{\partial \xi} d \xi d \eta+\frac{2}{\Delta z} \sum_{k=1}^{M} w_{k} \int_{-1}^{1} \int_{-1}^{1} P_{m} N_{n} \frac{\partial N_{k}}{\partial \eta} d \xi d \eta\right\}, \\
& \text { para } m=1, \ldots, M \text {. }
\end{aligned}
$$


Dividindo a equação (4-68) por $\frac{\Delta x_{i, j} \Delta z_{i, j}}{4}$ e reescrevendo-a na forma matricial, tem-se:

$$
\underline{A}^{i, j} \vec{E}^{i, j}=\vec{b}_{E}^{i, j}
$$

onde a matriz $\underline{A}^{i, j}$ é a matriz de expansão e é dada pela equação (4-30), $\vec{E}^{i, j}$ é o vetor de coeficientes da energia no elemento $i, j$ e $\vec{b}_{E}^{i, j}$ é o vetor resultante para a energia no elemento $i, j$.

$\mathrm{O} m$-ésimo componente do vetor resultante para o elemento $i, j$ é dado por:

$$
\begin{aligned}
& b_{E m, n}^{i, j}= \frac{1}{\Delta t} \sum_{n=1}^{M} E_{n}^{t-\Delta t} \alpha_{m, n}^{i, j}+4 K \sum_{n=1}^{M} T_{n}\left[\frac{1}{\Delta x_{i, j}^{2}}\left(C_{z m, n}^{i, j}-D_{x m, n}^{i j}\right)+\frac{1}{\Delta z_{i, j}^{2}}\left(C_{x m, n}^{i, j}-D_{z m, n}^{i j}\right)\right]+ \\
&+8 \mu\left\{\frac{1}{\Delta x_{i, j}^{2}} \sum_{n=1}^{M} u_{n} \sum_{k=1}^{M} u_{k} v i s c_{1 m, n, k}^{i, j}+\frac{1}{\Delta x_{i, j} \Delta z_{i, j}} \sum_{n=1}^{M} u_{n} \sum_{k=1}^{M} w_{k} v i s c_{2 m, n, k}^{i, j}+\right. \\
&\left.+\frac{1}{\Delta z_{i, j}^{2}} \sum_{n=1}^{M} w_{n} \sum_{k=1}^{M} w_{k} v i s c_{3 m, n, k}^{i, j}\right\}-\frac{4}{3} \mu\left[\frac{1}{\Delta x_{i, j}} \sum_{n=1}^{M} u_{n} \Omega+\frac{1}{\Delta z_{i, j}} \sum_{n=1}^{M} w_{n} \psi\right]- \\
&-2 \sum_{n=1}^{M} p_{n}\left[\frac{1}{\Delta x_{i, j}} \sum_{k=1}^{M} u_{k} v i S c_{4 m, n, k}^{i, j}\right. \\
&
\end{aligned}
$$

onde $\quad \alpha_{\mathrm{m}, \mathrm{n}}^{\mathrm{i}, \mathrm{j}}, C_{x \mathrm{~m}, \mathrm{n}}^{i, j}, C_{z \mathrm{~m}, \mathrm{n}}^{i, j} D_{x \mathrm{~m}, \mathrm{n}}^{i, j}$ e $D_{z \mathrm{~m}, \mathrm{n}}^{i, j} \quad$ são $\quad$ as integrais já definidas anteriormente e os parâmetros $v i s c_{m, n, k}^{i, j}, v i s c_{2} \underset{m, n, k}{i, j}, v i s c_{3, n, k}^{i, j}, v i s c_{m, n, k}^{i, j}$ e visc $5_{m, n, k}^{i, j}$ são definidos pelas seguintes expressões: 


$$
\begin{aligned}
\operatorname{visc}_{1, n, k}^{i j}= & \int_{-1-1}^{1} \int_{m}^{1} P_{m} \frac{\partial N_{n}}{\partial \xi} \frac{\partial N_{k}}{\partial \xi} d \xi d \eta=\int_{-1}^{1} \int_{-1}^{1} N_{m} \frac{\partial N_{n}}{\partial \xi} \frac{\partial N_{k}}{\partial \xi} d \xi d \eta+ \\
& +\bar{u}^{i, j} \frac{\Delta t}{\Delta x_{i, j}} \int_{-1-1}^{1} \int_{-1}^{1} \frac{\partial N_{m}}{\partial \xi} \frac{\partial N_{n}}{\partial \xi} \frac{\partial N_{k}}{\partial \xi} d \xi d \eta+\bar{w}^{i, j} \frac{\Delta t}{\Delta z_{i, j}} \int_{-1-1}^{1} \int_{-1}^{1} \frac{\partial N_{m}}{\partial \eta} \frac{\partial N_{n}}{\partial \xi} \frac{\partial N_{k}}{\partial \xi} d \xi d \eta ;
\end{aligned}
$$

$$
\begin{aligned}
\operatorname{visc}_{2}{ }_{m, n, k}^{i j}= & \int_{-1-1}^{1} \int_{-1}^{1} P_{m} \frac{\partial N_{n}}{\partial \xi} \frac{\partial N_{k}}{\partial \eta} d \xi d \eta=\int_{-1-1}^{1} \int_{-1}^{1} N_{m} \frac{\partial N_{n}}{\partial \xi} \frac{\partial N_{k}}{\partial \eta} d \xi d \eta+ \\
& +\bar{u}^{i, j} \frac{\Delta t}{\Delta x_{i, j}} \int_{-1-1}^{1} \int_{-1}^{1} \frac{\partial N_{m}}{\partial \xi} \frac{\partial N_{n}}{\partial \xi} \frac{\partial N_{k}}{\partial \eta} d \xi d \eta+\bar{w}^{i, j} \frac{\Delta t}{\Delta z_{i, j}} \int_{-1-1}^{1} \int_{-1}^{1} \frac{\partial N_{m}}{\partial \eta} \frac{\partial N_{n}}{\partial \xi} \frac{\partial N_{k}}{\partial \eta} d \xi d \eta
\end{aligned}
$$

$$
\begin{aligned}
v i s c_{3 m, n, k}^{i j}= & \int_{-1}^{1} \int_{-1}^{1} P_{m} \frac{\partial N_{n}}{\partial \eta} \frac{\partial N_{k}}{\partial \eta} d \xi d \xi=\int_{-1}^{1} \int_{-1}^{1} N_{m} \frac{\partial N_{n}}{\partial \eta} \frac{\partial N_{k}}{\partial \eta} d \xi d \xi+ \\
& +\bar{u}^{i, j} \frac{\Delta t}{\Delta x_{i, j}} \int_{-1-1}^{1} \int_{-1}^{1} \frac{\partial N_{m}}{\partial \xi} \frac{\partial N_{n}}{\partial \eta} \frac{\partial N_{k}}{\partial \eta} d \xi d \xi+\bar{w}^{i, j} \frac{\Delta t}{\Delta z_{i, j}} \int_{-1-1}^{1} \int_{-1}^{1} \frac{\partial N_{m}}{\partial \eta} \frac{\partial N_{n}}{\partial \eta} \frac{\partial N_{k}}{\partial \eta} d \xi d \xi d ;
\end{aligned}
$$

$$
\begin{aligned}
\operatorname{visc} 4 m, n, k^{i j}= & \int_{-1}^{1} \int_{-1}^{1} P_{m} N_{n} \frac{\partial N_{k}}{\partial \xi} d \xi d \eta=\int_{-1}^{1} \int_{-1}^{1} N_{m} N_{n} \frac{\partial N_{k}}{\partial \xi} d \xi d \eta+ \\
& +\bar{u}^{i, j} \frac{\Delta t}{\Delta x_{i, j}} \int_{-1}^{1} \int_{-1}^{1} \frac{\partial N_{m}}{\partial \xi} N_{n} \frac{\partial N_{k}}{\partial \xi} d \xi d \eta+\bar{w}^{i, j} \frac{\Delta t}{\Delta z_{i, j}} \int_{-1-1}^{1} \int_{-1}^{1} \frac{\partial N_{m}}{\partial \eta} N_{n} \frac{\partial N_{k}}{\partial \xi} d \xi d \eta ;
\end{aligned}
$$

e

$$
\begin{aligned}
\operatorname{visc}_{5 m, n, k}^{i j}= & \int_{-1}^{1} \int_{-1}^{1} P_{m} N_{n} \frac{\partial N_{k}}{\partial \eta} d \xi d \eta=\int_{-1}^{1} \int_{-1}^{1} N_{m} N_{n} \frac{\partial N_{k}}{\partial \eta} d \xi d \eta+ \\
& +\bar{u}^{i, j} \frac{\Delta t}{\Delta x_{i, j}} \int_{-1-1}^{1} \int_{-1}^{1} \frac{\partial N_{m}}{\partial \xi} N_{n} \frac{\partial N_{k}}{\partial \eta} d \xi d \eta+\bar{w}^{i, j} \frac{\Delta t}{\Delta z_{i, j}} \int_{-1-1}^{1} \int_{-1}^{1} \frac{\partial N_{m}}{\partial \eta} N_{n} \frac{\partial N_{k}}{\partial \eta} d \xi d \eta .
\end{aligned}
$$




\subsection{8 - Equação da temperatura}

A partir da equação integrada e pesada da temperatura, dada por (4-26), abrem-se as integrais nas direções $x$ e $z$, obtendo-se a seguinte equação:

$$
\iint_{x} P_{m} E d x d z-\iint_{x} P_{m} \rho c_{v} T d x d z=0
$$

O mesmo procedimento matemático adotado para as equações anteriores é utilizado neste caso, ou seja, faz-se a mudança de variáveis de $x$ e $z$ para $\xi$ e $\eta$ e introduz-se a expansão para as variáveis $E, \rho$ e $T$, dadas por (4-13), obtendo-se a seguinte expressão:

$$
\begin{aligned}
& c_{v} \sum_{n=1}^{M} T_{n} \sum_{k=1}^{M} \rho_{k} \int_{-1}^{1} \int_{-1}^{1} P_{m} N_{n} N_{k} d \xi d \eta=\sum_{n=1}^{M} E_{n} \int_{-1-1}^{1} \int_{-1}^{1} P_{m} N_{n} d \xi d \eta, \\
& \text { para } m=1, \ldots, M .
\end{aligned}
$$

Reescrevendo a equação anterior na forma matricial tem-se:

$$
\underline{A}_{T}^{i, j} \vec{T}^{i, j}=\vec{b}_{T}^{i, j}
$$

onde a matriz $\underline{A}_{T}^{i, j}$ é a matriz de expansão da variável $T$ para o elemento $i, j, \vec{T}^{i, j}$ é o vetor dos coeficientes da temperatura para o elemento $i, j$ e $\vec{b}_{T}^{i, j}$ é o vetor resultante para a temperatura no elemento $i, j$. Nota-se que a dimensão da matriz $\underline{A}_{p}^{i, j}$ é $(M \mathrm{x} M)$ e a dimensão dos vetores $\vec{T}^{i, j}$ e $\vec{b}_{T}^{i, j}(M \times 1)$. O elemento $(m, n)$ da matriz $\underline{A}_{T}^{i, j}$ é dado por:

$$
A_{T m, n}^{i, j}=c_{v} \sum_{k=1}^{M} \rho_{k} \theta_{m, n, k}^{i, j}
$$


O $m$-ésimo componente do vetor resultante para o elemento $i, j$ é dado pela expressão:

$$
b_{T m, n}^{i, j}=\sum_{n=1}^{M} E_{n} \alpha_{m, n}^{i, j} .
$$

Os parâmetros $\alpha_{m, n}^{i, j}$ e $\theta_{m, n, k}^{i, j}$ são as integrais já definidas anteriormente.

Salienta-se que, embora neste trabalho $c_{V}$ é considerado constante, a equação da temperatura pode ser desenvolvida facilmente para valores variados de calor específico.

Todas as integrais que aparecem nos parâmetros das equações para a solução de um escoamento dependem das funções de expansão, que se encontram listadas no Apêndice A.

Para uma melhor compreensão de como as funções de expansão são ajustadas nos elementos retangulares, de forma a definir funções de canto, de lado e de área, no item 4.3 é apresentado um exemplo unidimensional da aplicação destas funções, a fim de se ter uma idéia real do método proposto neste trabalho.

\section{3 - Funções de expansão}

Nesta seção são apresentadas e comparadas as funções de expansão utilizadas no método clássico dos elementos finitos e as funções hierárquicas para um problema unidimensional. Com essas funções são apresentadas as principais vantagens da utilização das funções de expansão hierárquicas.

São também apresentadas as funções de expansão hierárquicas utilizadas em problemas bidimensionais com malhas retangulares. Essas funções são geradas através de associações das funções de uma dimensão, como será visto. 


\subsection{1 - Funções de expansão clássicas}

No método de elementos finitos clássico, os coeficientes de expansão das variáveis são identificados com as mesmas em pontos específicos da malha. Essa identificação é utilizada amplamente na literatura que trata de elementos finitos e fornece um significado físico aos coeficientes de expansão das variáveis. A desvantagem dessa definição pode ser facilmente observada a partir das funções linear, quadrática e cúbica aplicadas para o caso de uma dimensão. A Figura 4.2 mostra um elemento unidimensional típico de dimensão $\Delta x$ com funções de expansão linear, quadrática e cúbica.
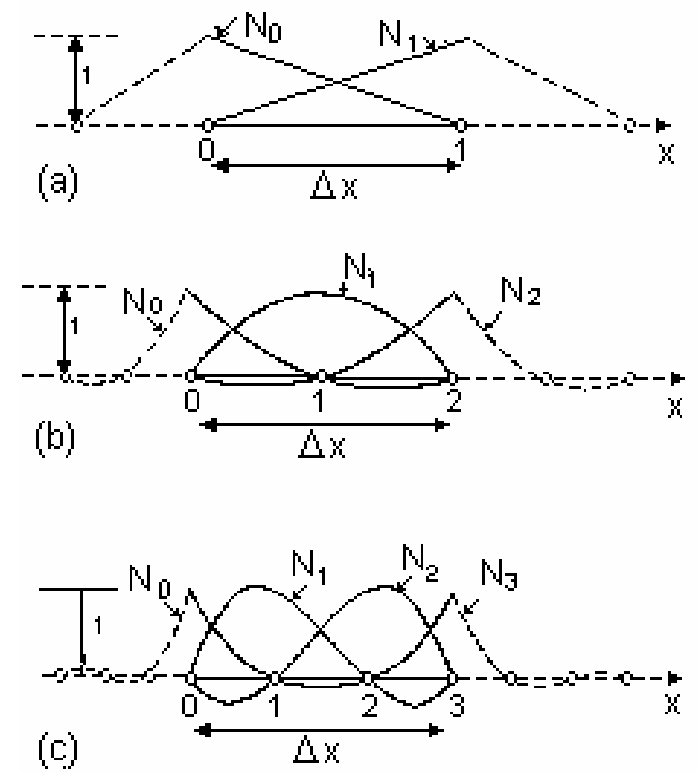

Figura 4.2 - Elementos unidimensionais e funções de expansão, (a) linear, (b) quadrática e (c) cúbica, segundo Zinkiewics e Morgan (1983).

Com elementos igualmente espaçados entre si, as funções de expansão utilizadas são as seguintes: 
Linear:

$$
N_{0}=-\frac{\xi-1}{2} ; \quad N_{1}=\frac{\xi+1}{2} .
$$

Quadrática

$$
N_{0}=\frac{\xi(\xi-1)}{2} ; \quad N_{1}=-(\xi-1)(\xi+1) ; \quad N_{2}=\frac{\xi(\xi+1)}{2}
$$

Cúbica:

$$
\begin{aligned}
& N_{0}=-\frac{9}{16}\left(\xi+\frac{1}{3}\right)\left(\xi-\frac{1}{3}\right)(\xi-1) ; \quad N_{1}=\frac{27}{16}(\xi+1)\left(\xi-\frac{1}{3}\right)(\xi-1) ; \\
& N_{2}=-\frac{27}{16}(\xi+1)\left(\xi+\frac{1}{3}\right)(\xi-1) ; N_{3}=\frac{9}{16}(\xi+1)\left(\xi+\frac{1}{3}\right)\left(\xi-\frac{1}{3}\right) .
\end{aligned}
$$

Nesses polinômios $\xi$ é a variável local, que dentro do intervalo $\Delta x$ varia de -1 a 1 . Observa-se que cada uma dessas funções está associada à variável no ponto da malha onde a respectiva função de expansão tem valor unitário. Por exemplo, no caso quadrático, a função $N_{0}(\xi)$ é igual a 1 para $\xi=-1$, ou no ponto 0 indicado na Figura 4.2, a função $N_{1}(\xi)$ é igual a 1 para $\xi=0$ (ponto 1), a função $N_{2}(\xi)$ é igual a 1 no ponto 2 e a função $N_{3}(\xi)$ é igual a 1 no ponto 3 .

A partir da Figura 4.2 e das equações (4-81), (4-82) e (4-83), pode-se observar que quanto mais se deseja refinar a solução pelo aumento do grau da expansão, é necessário reiniciar o problema em vista da mudança total e completa de todas as funções de expansão e das variáveis envolvidas.

A fim de exemplificar como é realizada a montagem dos elementos das matrizes de expansão, considera-se a equação da energia em uma dimensão. Para isso, reduz-se esta 
equação, equação (4-68), de duas para uma dimensão e considera-se que o fluido é invíscito $(\mu=0)$, então obtém-se a seguinte expressão:

$$
\begin{aligned}
\sum_{n=1}^{M} E_{n}\left\{\frac{\Delta x_{i}}{2 \Delta t} \int_{-1}^{1} P_{m} N_{n} d \xi+\sum_{k=1}^{M} u_{k} \int_{-1}^{1} P_{m} \frac{\partial N_{n} N_{k}}{\partial \xi} d \xi\right\}= \\
=\frac{\Delta x_{i}}{2 \Delta t} \sum_{n=1}^{M} E_{n}^{t-\Delta t} \int_{-1}^{1} P_{m} N_{n} d \xi+K \sum_{n=1}^{M} T_{n}\left[\int_{-1}^{1}\left(P_{m} \frac{\partial N_{n}}{\partial \xi}\right)_{\xi=-1}^{\xi=1} d \eta-\right. \\
\left.\left.-\frac{2}{\Delta x_{i}} \int_{-1}^{1} \frac{P_{m}}{\partial \xi} \frac{\partial N_{n}}{\partial \xi} d \xi\right]\right\} \\
\operatorname{para} m=1, \ldots, M .
\end{aligned}
$$

A fim de simplificar a montagem da matriz de expansão relativa à equação anterior, os termos temporais são desprezados e a velocidade é considerada constante e igual a $u$. Assim, a equação anterior reduz-se à seguinte expressão:

$$
\begin{aligned}
& \sum_{n=1}^{M} E_{n} \sum_{k=1}^{M} u_{k} \int_{-1}^{1} P_{m} \frac{\partial N_{n} N_{k}}{\partial \xi} d \xi=\frac{2 K}{\Delta x_{i}} \sum_{n=1}^{M} T_{n} \int_{-1}^{1} \frac{\partial P_{m}}{\partial \xi} \frac{\partial N_{n}}{\partial \xi} d \xi, \\
& \text { para } m=1, \ldots, M .
\end{aligned}
$$

Nota-se que, na medida em que a velocidade é considerada constante e igual a $u$, nos coeficientes correspondentes às funções de expansão, as velocidades são todas iguais a $u$.

Escrevendo a equação (4.85) na forma matricial, tem-se:

$$
\underline{A}_{E}^{i} \vec{E}^{i}=0 .
$$

O elemento $(m, n)$ da matriz de expansão é dado por: 


$$
A_{E m, n}^{i}=\sum_{k=1}^{M} u_{k} \int_{-1}^{1} P_{m} \frac{\partial N_{n} N_{k}}{\partial \xi} d \xi \text {, }
$$

onde $P_{m}$ é a função peso que é considerada igual ao polinômio de expansão $N_{m}$ a fim de simplificar o exemplo.

Calculando os elementos da matriz $\underline{A}_{E}^{i}$ conforme a equação (4-87), para as funções de expansão definidas pelas equações (4-81), (4-82) e (4-83), obtêm-se as matrizes para expansão linear, quadrática e cúbica, de acordo com o seguinte:

Linear

$$
\underline{A}_{E}^{i}=\left[\begin{array}{cc}
-\frac{1}{2} u & \frac{1}{2} u \\
-\frac{1}{2} u & \frac{1}{2} u
\end{array}\right] .
$$

Quadrática:

$$
\underline{A}_{E}^{i}=\left[\begin{array}{ccc}
-\frac{9}{10} u & \frac{2}{5} u & \frac{1}{10} u \\
\frac{2}{15} u & 0 & -\frac{2}{15} u \\
\frac{1}{30} u & -\frac{2}{5} u & \frac{9}{10} u
\end{array}\right] .
$$


Cúbica:

$$
A_{E}^{i}=\left[\begin{array}{cccc}
-\frac{83}{210} u & \frac{57}{80} u & -\frac{3}{10} u & \frac{7}{80} u \\
-\frac{793}{1120} u & 0 & \frac{81}{10} u & -\frac{657}{2240} u \\
\frac{69}{280} u & -\frac{81}{80} u & 0 & \frac{57}{80} u \\
-\frac{7}{80} u & \frac{3}{10} u & -\frac{57}{80} u & \frac{1}{2} u
\end{array}\right]
$$

Como pode ser visto, as matrizes de expansão mudam completamente quando se altera a ordem da expansão. Esse fato é mais um complicador, pois quando se deseja alterar o grau de expansão das variáveis deve-se, além de reiniciar o problema, calcular novamente as matrizes de expansão para todos os elementos.

\subsection{2 - Funções de expansão hierárquicas em uma dimensão}

No caso das funções de expansão hierárquicas, os coeficientes de expansão das variáveis não são identificados com as variáveis em pontos específicos da malha. Nesse caso, os coeficientes estão associados com as funções de expansão que são ajustadas nos elementos retangulares, definindo funções de canto, de lado e de área. Essa associação permite iniciar a solução de um problema com uma expansão linear e, se necessário, durante o processo de solução, adicionar novas funções de forma a aumentar o grau de expansão e obter uma solução de maior precisão. Essa característica pode ser facilmente observada através do desenvolvimento das funções de expansão hierárquicas apresentado a seguir.

As funções de expansão hierárquicas são baseadas em polinômios de Legendre. Os polinômios de Legendre formam um conjunto de funções com propriedades de ortogonalidade dentro do intervalo $-1 \leq \xi \leq 1$. O polinômio de Legendre de grau $m, L(\xi)$, pode ser gerado pela seguinte fórmula de recorrência, conforme Zienkiewicz e Morgan (1983): 


$$
N_{m}(\xi)=\frac{1}{(m-1) ! 2^{m-1}} \frac{1}{d \xi^{m}}\left[\left(\xi^{2}-1\right)^{m}\right] .
$$

Por exemplo, integrando-se a expressão acima para $m=2,3,4$ e 5 obtêm-se os seguintes polinômios:

$$
\begin{array}{ll}
N_{2}=-\left(\xi^{2}-1\right) ; & N_{4}=\frac{1}{4}\left(15 \xi^{4}-18 \xi^{2}+3\right) ; \\
N_{3}=2\left(\xi^{3}-\xi\right) ; & N_{5}=7 \xi^{5}-10 \xi^{3}+3 \xi .
\end{array}
$$

Essas funções correspondem às funções de expansão de grau 2, 3, 4 e 5 para um elemento unidimensional. As funções de expansão lineares utilizadas em conjunto com essas funções de grau superior são as mesmas utilizadas no método dos elementos finitos clássicos, ou seja, as funções presentes na equação (4-81). O comportamento dessas funções, até o terceiro grau, no intervalo $-1 \leq \xi \leq 1$, pode ser visto na Figura 4.3.

(a)

(b)

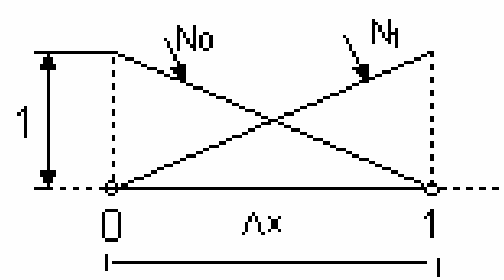

(c)
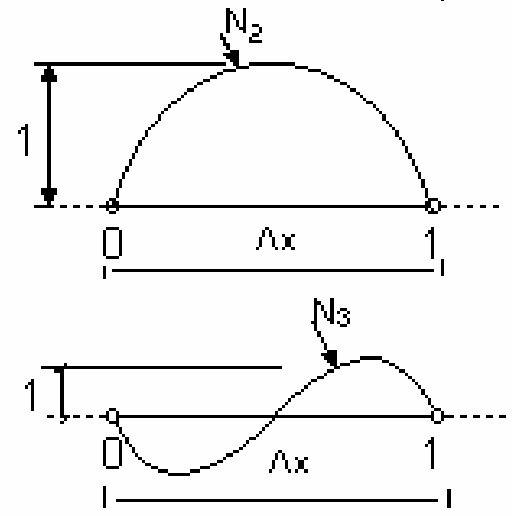

Figura 4.3 - Elementos hierárquicos e funções de expansão quase ortogonais, (a) linear, (b) quadrática e (c) cúbica. 
Observa-se que as funções lineares $N_{0}(\xi)$ e $N_{1}(\xi)$ estão associadas,respectivamente, à variável física nos pontos 0 e 1 do elemento, da mesma forma que no caso do método dos elementos finitos clássico. Contudo, as funções de expansão de grau 2, ou superiores, não estão associadas à variável física em um ponto determinado da malha. Essas funções alteram o valor da variável ao longo de todo o elemento, exceto nos pontos 0 e 1 , onde assumem valor igual a zero. A utilização dessas funções permite introduzir ou retirar uma função de expansão e seu respectivo coeficiente (a menos das funções lineares), durante o processo de solução de forma relativamente fácil.

Considerando-se a equação de energia em uma dimensão, cujos elementos da matriz de expansão para um elemento são dados pela equação (4-87), pode-se obter as matrizes de expansão para expansões lineares, quadráticas e cúbicas com as funções hierárquicas. Notase que, nesse caso, para se ter velocidade $u$ constante, tem-se que os coeficientes correspondentes à velocidade para as funções de expansão $N_{0}(\xi)$ e $N_{1}(\xi)$ é igual a $u$ e para as outras funções é igual a zero. Assim, as matrizes de expansão resultam em:

Linear:

$$
\underline{A}_{E}^{i}=\left[\begin{array}{cc}
-\frac{1}{2} u & \frac{1}{2} u \\
-\frac{1}{2} u & \frac{1}{2} u
\end{array}\right]
$$

Quadrática:

$$
\underline{A}_{E}^{i}=\left[\begin{array}{ccc}
-\frac{1}{2} u & \frac{1}{2} u & \frac{2}{3} u \\
-\frac{1}{2} u & \frac{1}{2} u & -\frac{2}{3} u \\
-\frac{2}{3} u & \frac{2}{3} u & 0
\end{array}\right] .
$$


Cúbica:

$$
\underline{A}_{E}^{i}=\left[\begin{array}{cccc}
-\frac{1}{2} u & \frac{1}{2} u & \frac{2}{3} u & 0 \\
-\frac{1}{2} u & \frac{1}{2} u & -\frac{2}{3} u & 0 \\
-\frac{2}{3} u & \frac{2}{3} u & 0 & -\frac{16}{15} u \\
0 & 0 & \frac{16}{15} u & 0
\end{array}\right]
$$

Nesse caso, pode-se ver que as matrizes não se alteram quando se muda a ordem da expansão, ou seja, não há a necessidade de se recalcular os elementos das matrizes do estágio anterior. Isso, aliado ao fato de se poder alterar o grau de expansão das variáveis sem a necessidade de reiniciar o problema é a grande vantagem das funções de expansão hierárquicas.

\subsection{3 - Funções de expansão hierárquicas em duas dimensões}

Com base nas funções hierárquicas em uma dimensão, a geração das funções de expansão hierárquicas em duas dimensões para elementos retangulares torna-se simples. A Figura 4.4 mostra um elemento retangular, com os seus cantos numerados de 1 a 4 , e o perfil de algumas funções de expansão associadas ao canto 3 e ao lado 3-4. 


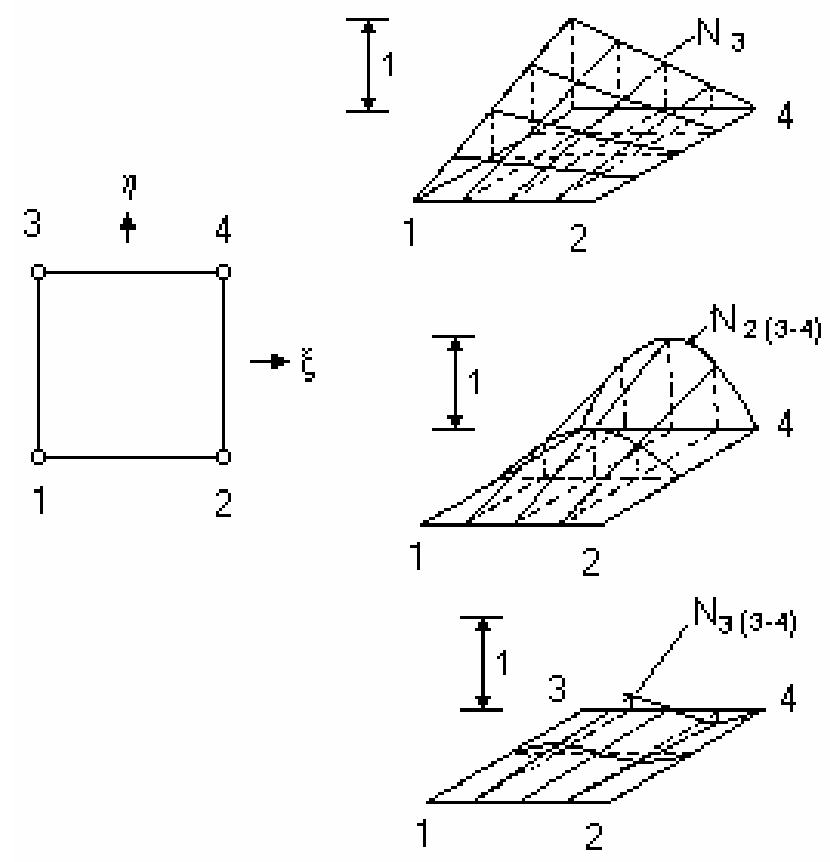

Figura 4.4 - Elemento retangular e algumas funções de expansão hierárquicas associadas.

As funções lineares associadas aos cantos dos elementos são obtidas pelo produto de duas funções lineares, da forma da equação (4-81), sendo cada uma, função de uma direção. Por exemplo, a função de expansão linear associada ao canto 3 é dada por:

$$
N_{3}(\xi, \eta)=-\frac{1}{4}(\xi-1)(\eta+1)
$$

Nota-se que essa função é igual a 1 no canto 3 e igual a zero nos outros três cantos.

As funções associadas aos cantos dos elementos são funções lineares e os coeficientes associados a estas funções representam o valor físico da variável naquela posição. Observa-se, também, que o coeficiente associado a uma determinada função de canto está presente em quatro elementos distintos e é o mesmo para estes quatro elementos. 
As funções de expansão associadas aos lados dos elementos são obtidas pelo produto de uma função linear unidimensional, ao longo de uma direção, por uma função de grau 2 ou superior ao longo da outra direção. Assim, por exemplo, as funções de expansão de graus 2 e 3, associadas ao lado (3-4) do elemento da Figura 4.4 são, respectivamente, as seguintes:

$$
\begin{aligned}
& N_{2(3-4)}(\xi, \eta)=-\frac{1}{2}(\eta+1)\left(\xi^{2}-1\right) ; \mathrm{e} \\
& N_{3(3-4)}(\xi, \eta)=(\eta+1)\left(\xi^{3}-\xi\right) .
\end{aligned}
$$

Observa-se que o valor dessas funções é zero em todos os cantos e nos outros três lados. Os coeficientes associados às funções de lado não representam a variável física em nenhuma posição determinada. Esses coeficientes estão simplesmente associados à forma geométrica da respectiva função. Ressalta-se que cada um desses coeficientes pertence a dois elementos adjacentes.

A identificação dos coeficientes de expansão associados com qualquer um dos lados do elemento e com os cantos deste lado, com o mesmo valor no elemento adjacente, garante automaticamente a singularidade da variável ao longo daquela lateral e, com isso, a continuidade desta variável no domínio da solução é assegurada.

As funções de expansão associadas à área de um elemento podem ser obtidas através do produto de duas funções, uma na variável $\xi$ e outra na variável $\eta$, de graus maiores do que 1. Por exemplo, a função de grau 2, para cada direção, é dada por:

$$
N_{2(\text { área })}(\xi, \eta)=\left(\xi^{2}-1\right)\left(\eta^{2}-1\right) .
$$


A equação (4-98) fornece uma função de expansão que é associada a um parâmetro que não está conectado a mais de um elemento, isto é, um parâmetro que é interno a um único elemento. Observa-se que o valor dessa função é zero nos quatro cantos e nos quatro lados do elemento.

As funções de expansão para os outros cantos e para os outros lados são obtidas de forma similar. O Apêndice A apresenta, com detalhes, todas as funções de expansão associadas a um elemento, até o grau 6. Embora o desenvolvimento apresentado neste trabalho tenha limitado o grau do polinômio em até 6, nada impede que sejam acrescidos graus maiores, quando for exigido um detalhamento maior na solução do problema que estiver sendo analisado.

\section{4 - Processo de agrupamento das equações dos elementos}

No item 4.2 foram desenvolvidas as equações de conservação utilizando o Método de Expansão em Funções Hierárquicas para cada elemento da malha. Cada uma das equações de conservação transforma-se, para cada elemento, em uma equação matricial de $M$ equações, onde $M$ é o número de funções de expansão utilizadas para descrever as variáveis no elemento.

A Figura 4.5 apresenta um elemento e os coeficientes de expansão de uma variável genérica $\phi$ associados com os cantos, lados e área. 


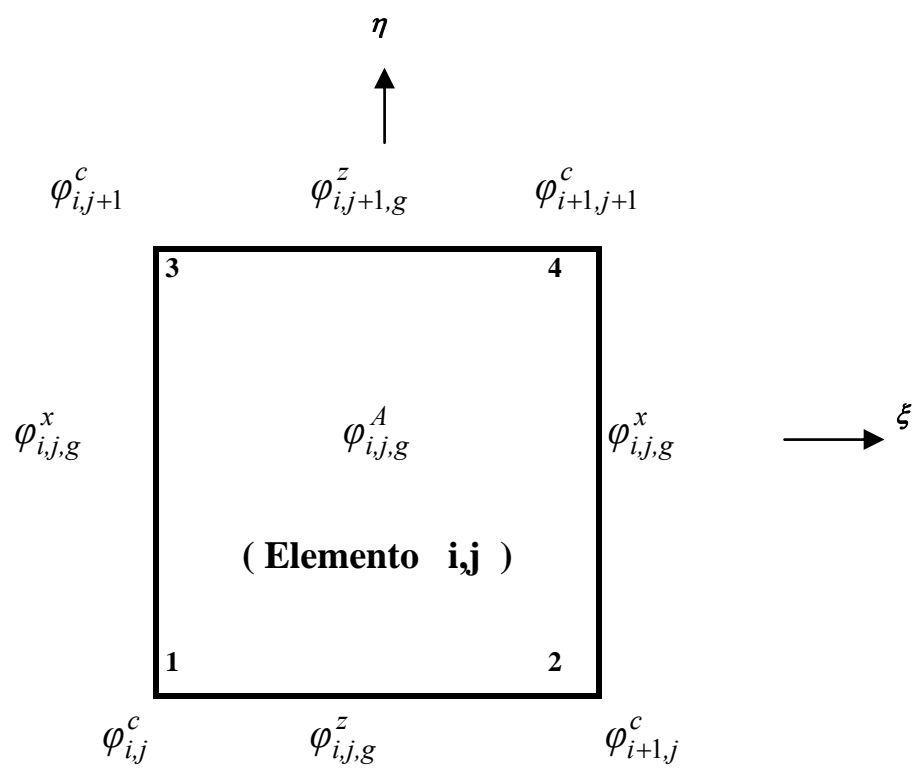

Figura 4.5 - Elemento retangular de duas dimensões e seus parâmetros associados.

Na Figura 4.5, os coeficientes $\phi_{i, j}^{c}$ são os parâmetros de canto associados às funções de expansão lineares, $\phi_{i, j, g}^{x}$ e $\phi_{i, j, g}^{z}$ são os coeficientes de lado associados às funções de expansão de lado, e $\phi_{i, j, g}^{A}$ são os coeficientes de área associados às funções de expansão de área. O subscrito $g$, presente nos coeficientes de lado e de área, representa o grau da função de expansão associada a esse coeficiente.

Pode-se observar na Figura 4.5 que os coeficientes de canto pertencem a quatro elementos diferentes e os coeficientes de lado pertencem a dois elementos. Dessa forma, esses coeficientes estão presentes nas equações de mais de um elemento. Para calcular os coeficientes que pertencem a mais de um elemento, deve-se usar as equações de conservação de todos os elementos nos quais o coeficiente está presente. Assim, a equação final correspondente a um coeficiente de expansão da malha é constituída por equações referentes a diversos elementos. O processo de obtenção das equações finais associadas a cada coeficiente de expansão da malha é chamado de processo de agrupamento. 
A fim de explicar como é realizado o processo de agrupamento para gerar as equações finais, cuja solução fornece os coeficientes de expansão, são considerados quatro elementos adjacentes da malha, como mostra a Figura 4.6.

Observa-se que na Figura 4.6 os coeficientes de área não estão representados, pois na medida em que pertencem a um único elemento, as equações referentes a estes coeficientes não entram no processo de agrupamento.
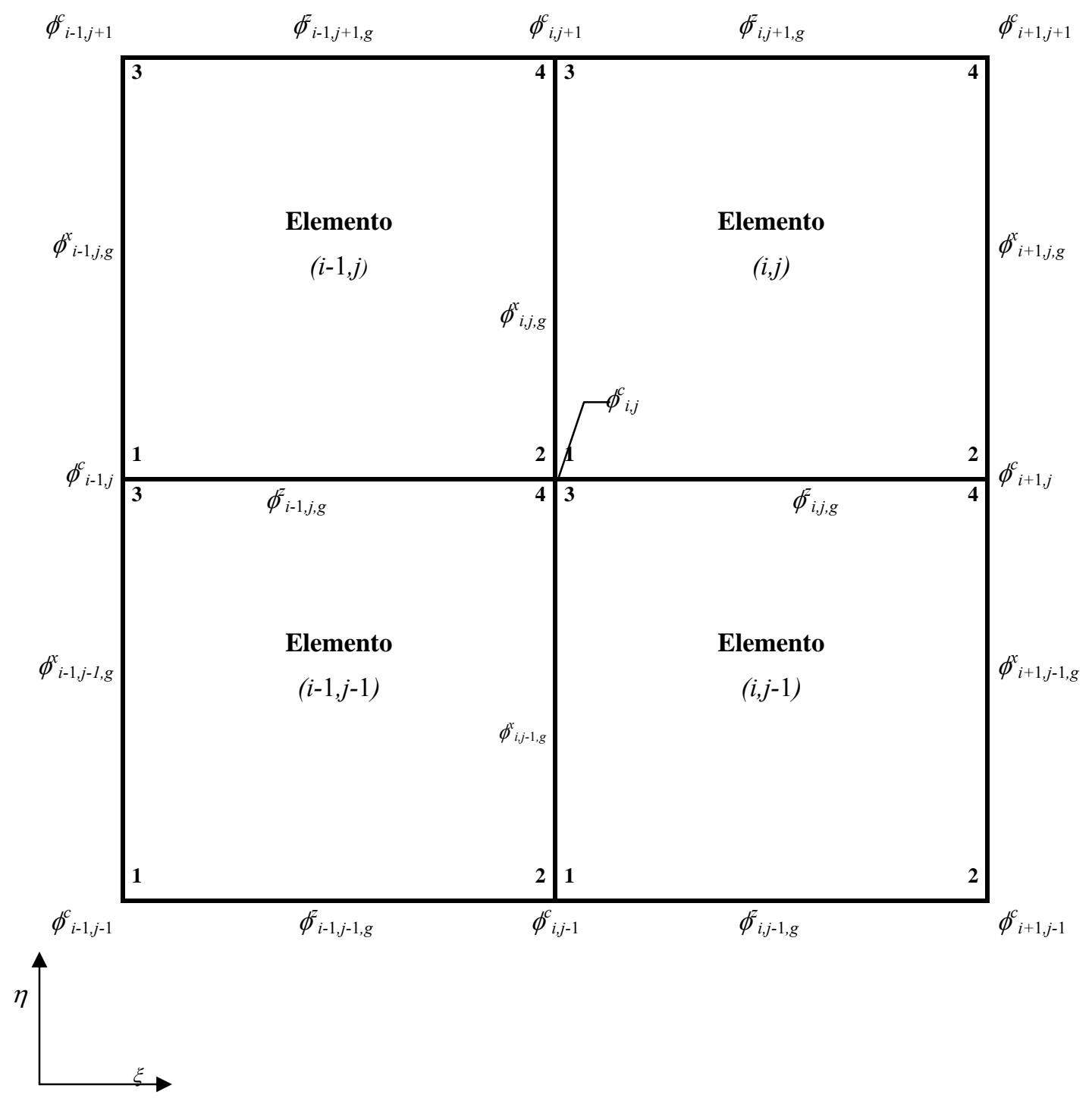

Figura 4.6 - Quatro elementos adjacentes e seus coeficientes de expansão associados. 
Pela Figura 4.6 observa-se que o coeficiente $\phi_{i, j}^{c}$ está associado à função de expansão $N_{1}$ do elemento $(i, j)$, mas também está associado à função $N_{2}$ no elemento $(i-1, j)$, à função $N_{3}$ no elemento $(i, j-1)$ e à função $N_{4}$ no elemento $(i-1, j-1)$. Portanto, como se nota, os coeficientes de canto estão conectados a quatro diferentes elementos através de quatro diferentes funções de expansão. Os coeficientes de lado $\phi_{i, j, g}^{x}$ estão conectados a dois elementos, aos elementos $(i, j)$ através da função de expansão $N_{L(1-3)}^{g}$, e ao elemento $(i-1, j)$, através da função $N_{L(2-4)}^{g}$. Os coeficientes de lado $\phi_{i, j, g}^{z}$ estão associados à função de expansão $N_{L(1-2)}^{g}$ do elemento $(i, j)$ e à função $N_{L(3-4)}^{g}$ do elemento $(i, j-1)$. Portanto, os coeficientes de lado estão conectados a dois elementos diferentes através de duas funções de expansão.

Como já visto, cada equação de conservação gera $M$ equações para cada elemento e a $m$-ésima equação está associada à $m$-ésima função de expansão.

Para exemplificar o processo de agrupamento considera-se uma expansão linear. Nota-se que na expansão linear somente existem os coeficientes de canto. Assim, a equação de conservação da variável $\phi$, escrita na forma matricial, para os elementos $(i, j),(i-1, j),(i, j-$ 1) e $(i-1, j-1)$ gera as seguintes expressões:

$$
\begin{array}{ll}
\underline{A}^{i, j} \vec{\varphi}=\vec{b}^{i, j} & \text { elemento }(i, j) ; \\
\underline{A}^{i-1, j} \vec{\varphi}=\vec{b}^{i-1, j} & \text { elemento }(i-1, j) ; \\
\underline{A}^{i, j-1} \vec{\varphi}=\vec{b}^{i, j-1} & \text { elemento }(i, j-1) ; \mathrm{e} \\
\underline{A}^{i-1, j-1} \vec{\varphi}=\vec{b}^{i-1, j-1} & \text { elemento }(i-1, j-1),
\end{array}
$$

onde $\vec{\phi}$ é o vetor que contém os coeficientes de canto dos respectivos elementos. 
Para a expansão linear, cada uma dessas equações matriciais consiste de quatro equações algébricas lineares. Para obter a equação final do coeficiente $\phi_{i, j}^{c}$ deve-se utilizar as equações correspondentes a este coeficiente nos quatro elementos envolvidos. Assim, para o coeficiente $\phi_{i, j}^{c}$ utiliza-se a primeira equação da equação matricial (4-99) do elemento $(i, j)$, a segunda equação do sistema de equações (4-100) do elemento $(i-1, j)$, a terceira equação do elemento $(i, j-1)$ e a quarta equação do elemento $(i-1, j-1)$, descritas a seguir:

$$
\begin{aligned}
& a_{11}^{i, j} \phi_{i, j}^{c}+a_{12}^{i, j} \phi_{i+1, j}^{c}+a_{13}^{i, j} \phi_{i, j+1}^{c}+a_{14}^{i, j} \phi_{i+1, j+1}^{c}=b_{1}^{i, j} \\
& a_{21}^{i-1, j} \phi_{i-1, j}^{c}+a_{22}^{i-1, j} \phi_{i, j}^{c}+a_{23}^{i-1, j} \phi_{i-1, j+1}^{c}+a_{24}^{i-1, j} \phi_{i, j+1}^{c}=b_{2}^{i-1, j} \\
& a_{31}^{i, j-1} \phi_{i, j-1}^{c}+a_{32}^{i, j-1} \phi_{i+1, j-1}^{c}+a_{33}^{i, j-1} \phi_{i, j}^{c}+a_{34}^{i, j-1} \phi_{i+1, j}^{c}=b_{3}^{i, j-1} ; \mathrm{e} \\
& a_{41}^{i-1, j-1} \phi_{i-1, j-1}^{c}+a_{42}^{i-1, j-1} \phi_{i, j-1}^{c}+a_{43}^{i-1, j-1} \phi_{i-1, j}^{c}+a_{44}^{i-1, j-1} \phi_{i, j}^{c}=b_{4}^{i-1, j-1},(4-1)
\end{aligned}
$$

onde os parâmetros $a_{m, n}^{i, j}$ são os componentes das matrizes de expansão dos elementos da malha envolvidos com o coeficiente $\phi_{i, j}^{c}$ e $b_{m}^{i, j}$ são os componentes do vetor resultante. Os parâmetros $a_{m, n}^{i, j}$ e $b_{m}^{i, j}$ são constituídos por integrais das funções de expansão, por parâmetros geométricos dos elementos, por propriedades físicas do fluido e também por componentes da velocidade no fluido no elemento, na forma derivada no item 4.2.

A equação final do coeficiente $\phi_{i, j}^{c}$ é obtida simplesmente somando-se as equações (4-103), (4-104), (4-105) e (4-106), resultando em: 


$$
\begin{aligned}
& \phi_{i, j}^{c}\left(a_{11}^{i, j}+a_{22}^{i-1, j}+a_{33}^{i, j-1}+a_{44}^{i-1, j-1}\right)+\phi_{i+1, j}^{c}\left(a_{12}^{i, j}+a_{34}^{i, j-1}\right)+ \\
& +\phi_{i, j+1}^{c}\left(a_{13}^{i, j}+a_{24}^{i-1, j}\right)+\phi_{i-1, j}^{c}\left(a_{21}^{i-1, j}+a_{43}^{i-1, j-1}\right)+\phi_{i, j-1}^{c}\left(a_{31}^{i, j-1}+a_{42}^{i-1, j-1}\right)+ \\
& +\phi_{i+1, j+1}^{c} a_{14}^{i, j}+\phi_{i-1, j+1}^{c} a_{23}^{i-1, j}+\phi_{i+1, j-1}^{c} a_{32}^{i, j-1}+\phi_{i-1, j-1}^{c} a_{41}^{i-1, j-1}= \\
& =b_{1}^{i, j}++b_{2}^{i-1, j}+b_{3}^{i, j-1}+b_{4}^{i-1, j-1}
\end{aligned}
$$

A obtenção das equações finais para os coeficientes de lado é realizada de forma semelhante, porém somente dois elementos da malha são utilizados. Assim, por exemplo, para obter a equação final correspondente ao coeficiente de expansão $\phi_{i, j, g}^{x}$, utilizam-se as equações de conservação dos elementos $(i, j)$ e $(i-1, j)$.

A obtenção de equações similares à equação (4-107) para cada um dos coeficientes de expansão da malha consiste no processo de agrupamento. A solução dessa equação, em conjunto com as equações correspondentes aos outros coeficientes de expansão, fornece a solução do problema desejado.

As equações de conservação desenvolvidas no item 4.2 e o processo de agrupamento foram implementados no programa computacional desenvolvido neste trabalho, denominado Programa do Método da Expansão em Funções Hierárquicas em duas Dimensões - PMEFH2D.

\section{5 - Algoritmo de solução}

No algoritmo de solução utilizado para resolver o conjunto de equações de NavierStokes em duas dimensões para fluidos compressíveis em alta velocidade, deve-se considerar que todas as equações têm que ser resolvidas simultaneamente. No entanto, é utilizado um processo de duas etapas iterativas, onde se tem uma iteração interna e uma iteração externa. $\mathrm{Na}$ iteração interna são calculadas as variáveis $\rho, G_{x}, G_{z}, u, w$ e $p$ e na 
iteração externa é realizado o cálculo de $E$ e $T$. O processo iterativo, em cada intervalo de tempo de integração, é realizado segundo os seguintes passos:

1) Incremento do tempo;

2) Iteração interna reduzida com o objetivo das variáveis convergirem mais rapidamente;

a) calcular $\rho$;

b) calcular $G_{x}$ e $G_{z}$;

c) obter $u$ e $w$;

d) calcular $p$;

e) interagir entre os itens (a) e (d) até determinar a massa específica, as componentes da velocidade, $u$ e $w$, da velocidade mássica $G_{x}$ e $G_{z}$, e a pressão, dentro da precisão desejada;

3) Calcular $E$ e $T$, utilizando os valores das variáveis obtidas no processo do item (2);

4) Voltar para o ítem (2);

5) Repetir itens (2) e (3) até a convergência desejada das variáveis dentro da precisão desejada; e

6) Finalmente avançar para um novo nível de tempo, retornando ao item (1). 


\section{5 - RESULTADOS}

Este capítulo apresenta os casos estudados com o método da expansão em funções hierárquicas na solução das equações de Navier-Stokes, para problemas de escoamento, em duas dimensões, de fluidos compressíveis em alta velocidade.

A fim de analisar e validar o método numérico proposto neste trabalho, foram simulados alguns casos conhecidos da literatura. Esses casos são os seguintes: teste de continuidade, teste de consistência e estabilidade, problema do degrau de temperatura e problema do choque oblíquo.

A partir da aplicação do método numérico proposto para a solução das equações de conservação, como descrito no capítulo 4, elaborou-se um programa computacional para simular e poder analisar os testes e problemas de mecânica dos fluidos, acima citados, denominado PMEFH2D e, consequentemente, poder validar o método numérico desenvolvido neste trabalho.

O fluido de trabalho utilizado para testar o método numérico desenvolvido foi o ar, considerando a sua viscosidade $\mu=2.10^{-5} \mathrm{~kg} / \mathrm{ms}$ e a sua condutividade térmica $\mathrm{k}=2.10^{-2} \mathrm{~J} / \mathrm{msK}$, constantes. Quanto à discretização espacial, o método numérico desenvolvido neste trabalho empregou malhas estruturadas constituídas de elementos retangulares.

\section{1 - TESTE DE CONTINUIDADE}

Este teste foi realizado, basicamente, para verificar se o programa computacional, desenvolvido para simular o conjunto de equações diferenciais apresentadas no capítulo 4, interfere na solução dessas equações, isto é, verificar se o programa gera algum tipo de perturbação indesejável não prevista pelo método numérico. 
Para isso, simulou-se o escoamento de um fluido (ar), à alta velocidade, onde as condições de contorno do problema são dadas apenas à montante do escoamento. Inicialmente, com o escoamento na direção $x$, da esquerda para a direita, figura 5.1. Depois, com o escoamento na direção $z$, de baixo para cima, figura 5.2 e, finalmente, com o escoamento em uma direção formando um ângulo de $45^{\circ}$ com a direção $x$, figura 5.3.

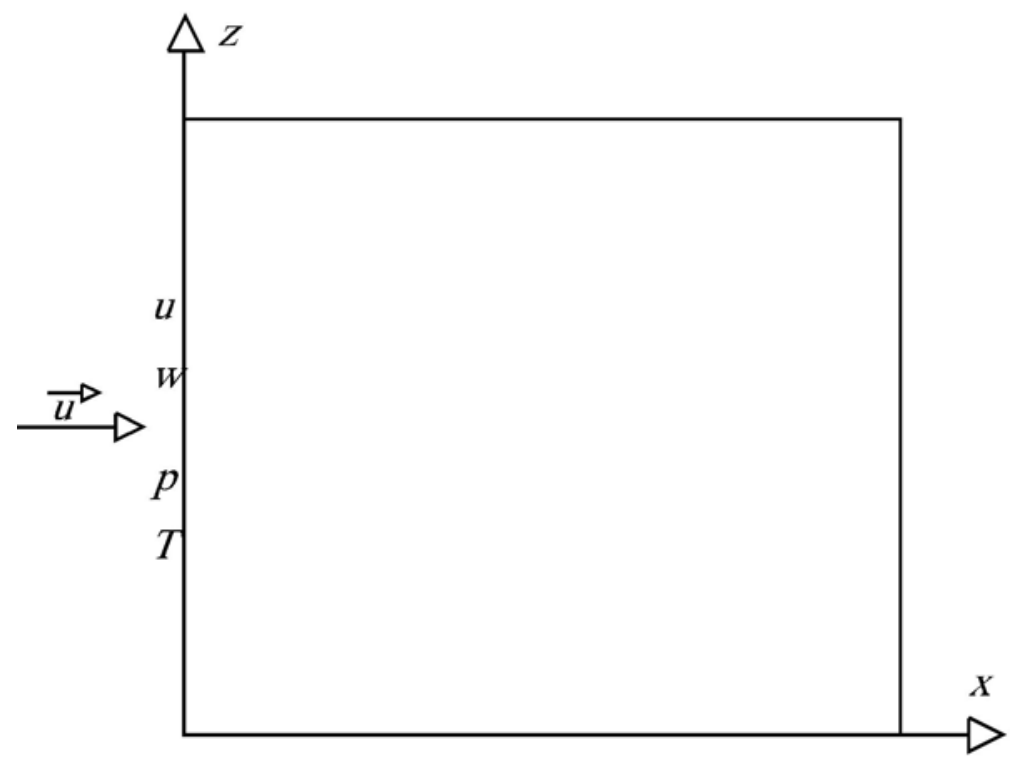

Figura 5.1 - Domínio computacional (0,5 m x 0,5 m) - Escoamento de ar à alta velocidade na direção $x$ da esquerda para a direita. 


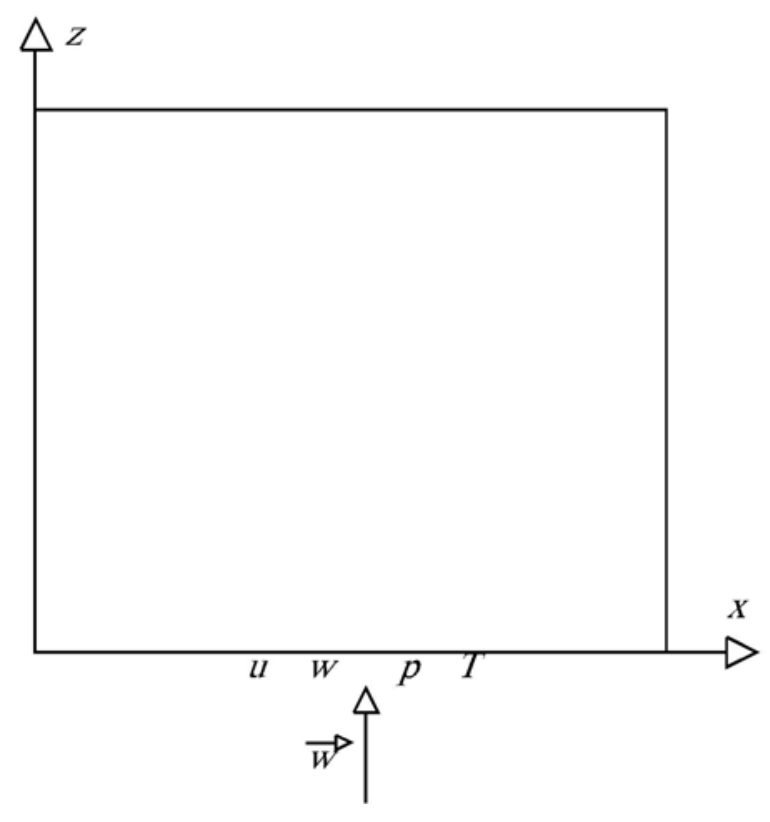

Figura 5.2 - Domínio computacional (0,5 m x 0,5 m) - Escoamento de ar à alta velocidade na direção $z$ de baixo para cima.

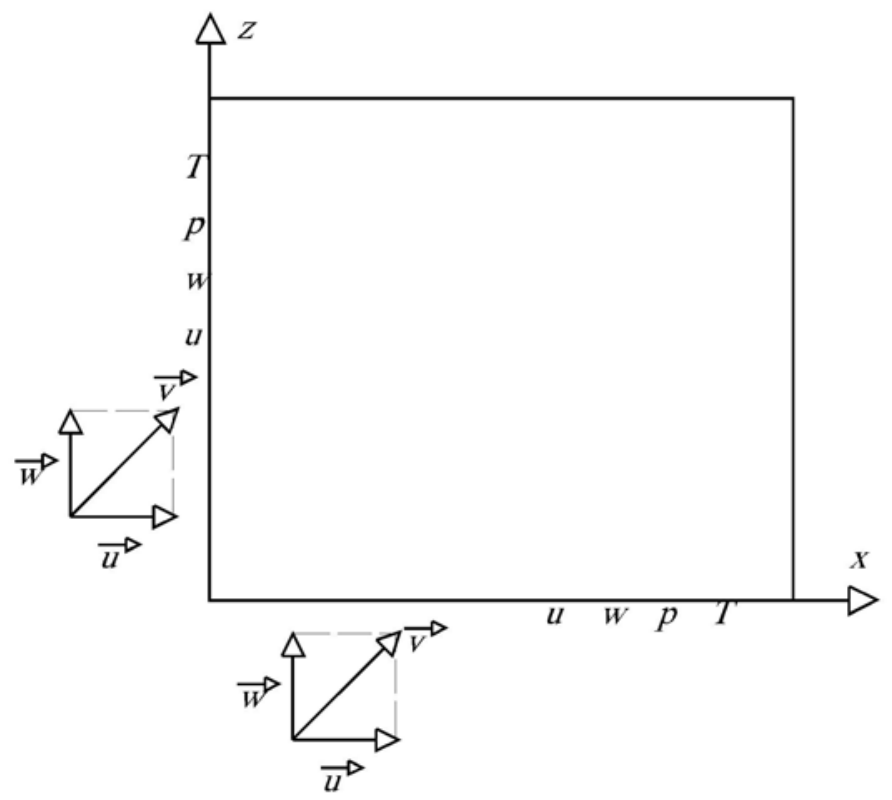

Figura 5.3 - Domínio computacional (0,5 m x 0,5 m) - Escoamento de ar à alta velocidade nas direções $x$ e $z$ com ângulo de inclinação igual a $45^{\circ}$. 
Inicialmente, para o teste referente à figura 5.1, tem-se as seguintes condições de contorno fornecidas na face esquerda do domínio computacional: velocidade na direção do eixo $x, u=1000 \mathrm{~m} / \mathrm{s}$; velocidade na direção do eixo $z, w=0$; temperatura do fluido $T=300 \mathrm{~K}$ e pressão $p=1$ bar.

Para o teste referente à figura 5.2, tem-se as seguintes condições de contorno fornecidas na face inferior do domínio computacional: velocidade na direção $x, u=0$; velocidade na direção $z, w=1000 \mathrm{~m} / \mathrm{s}$; temperatura do fluido, $T=300 \mathrm{~K}$ e pressão $p=1$ bar.

Finalmente, para o teste referente à figura 5.3, tem-se as seguintes condições de contorno fornecidas nas faces esquerda e inferior do domínio computacional: velocidade na direção $x, u=1000 \mathrm{~m} / \mathrm{s}$; velocidade na direção $z, w=1000 \mathrm{~m} / \mathrm{s}$; temperatura do fluido $T=300 \mathrm{~K}$ e pressão $p=1$ bar.

Os dados gerais da malha utilizada são:

- comprimento das células nas direções $x$ e $z$ igual a 0,05m;

- número de células nas direções $x$ e $z$ igual a 10;

- grau de expansão dos polinômios igual a 2; e

- intervalo de tempo igual a $10^{-5} \mathrm{~s}$.

De acordo com as condições de contorno descritas acima, são mostrados a seguir alguns resultados obtidos. 


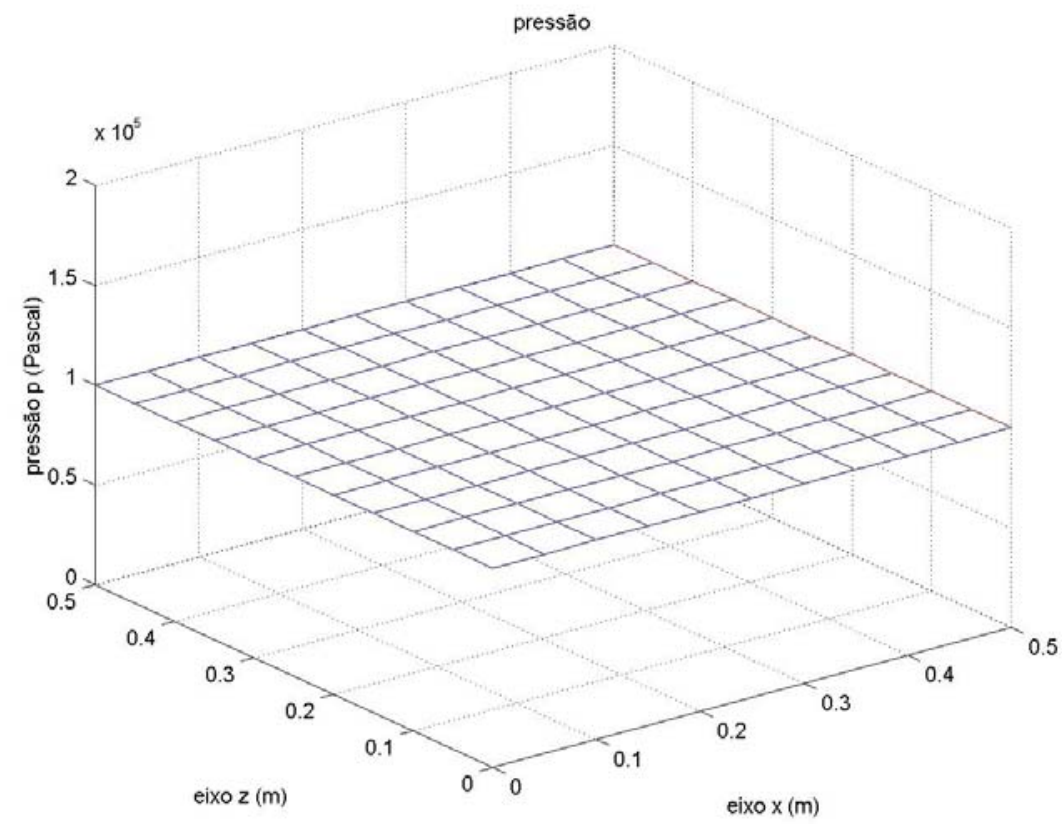

Figura 5.4 - Comportamento da pressão referente ao domínio computacional da figura 5.1.

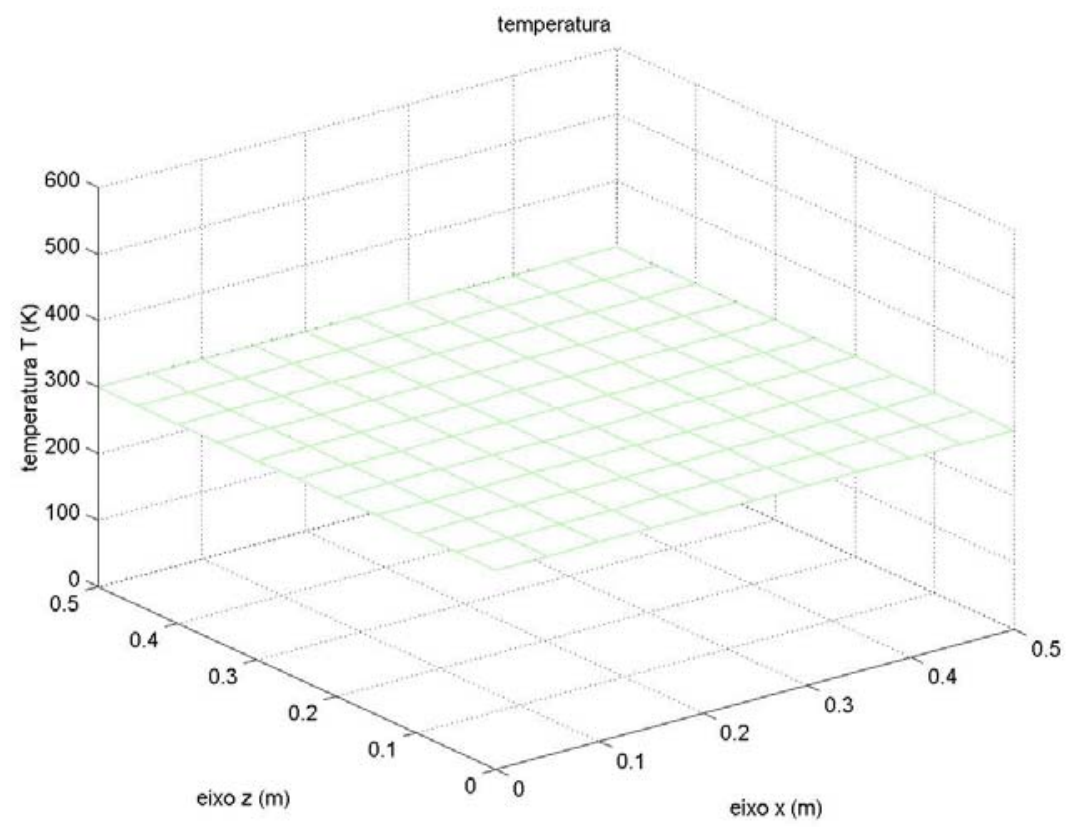

Figura 5.5 - Comportamento da temperatura referente ao domínio computacional da figura 5.2. 


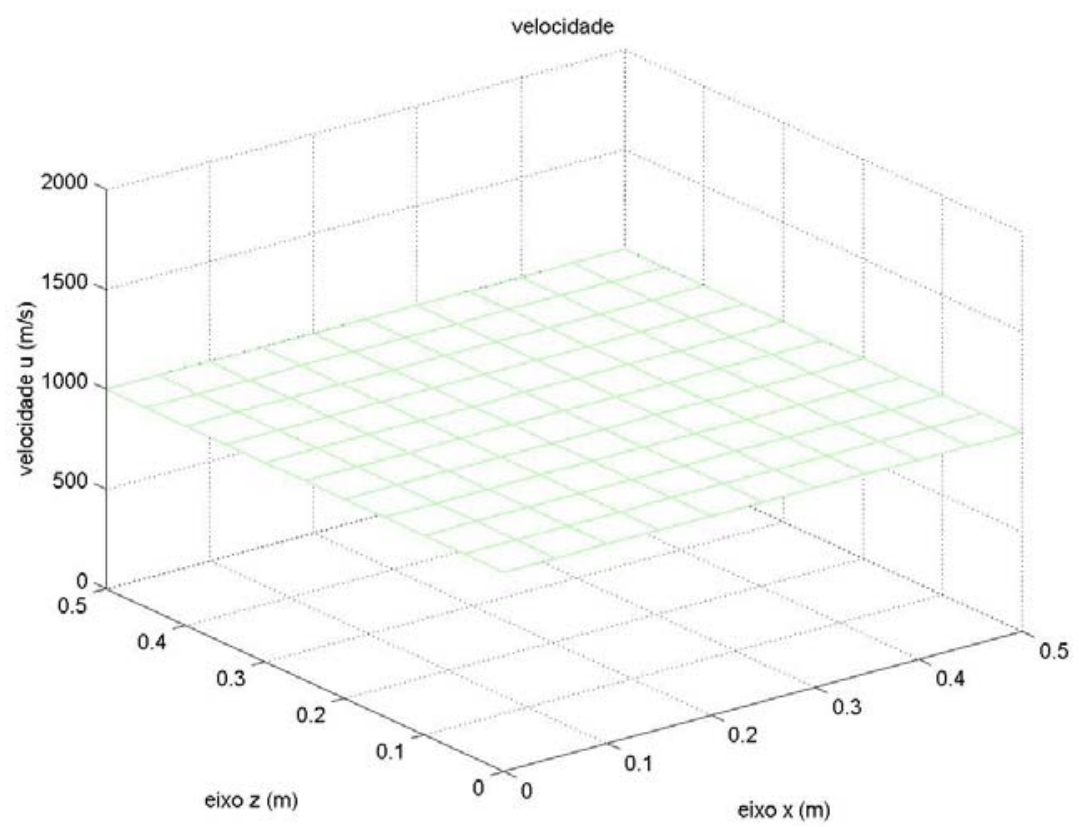

Figura 5.6 - Comportamento da velocidade $u$ referente ao domínio computacional da figura 5.3.

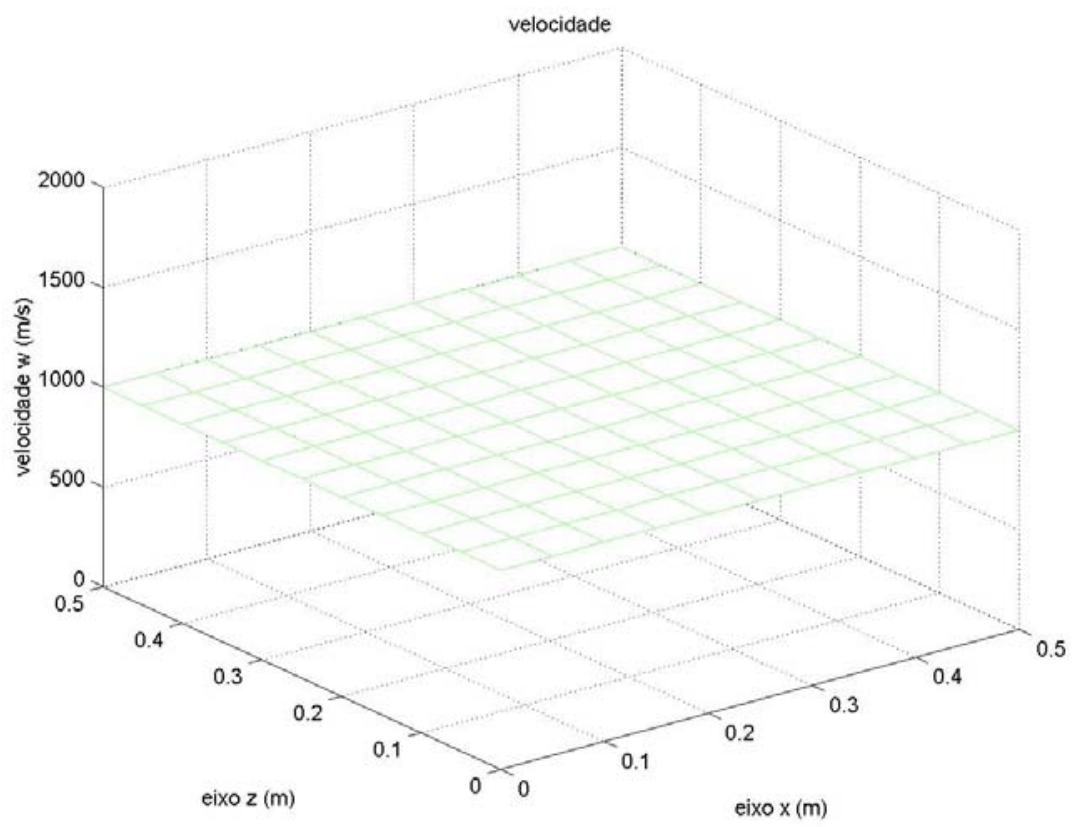

Figura 5.7 - Comportamento da velocidade $w$ referente ao domínio computacional da figura 5.3. 
Nota-se que, em todos os casos estudados, as grandezas físicas analisadas velocidade na direção $x(u)$, velocidade na direção $z(w)$, pressão $(p)$ e temperatura $(T)$, convergem para o valor esperado, sem perturbações aparentes.

\section{2 - TESTE DE CONSISTÊNCIA E ESTABILIDADE}

O segundo teste realizado consiste na verificação da consistência e estabilidade do método numérico, através da simulação de uma onda de choque normal de um escoamento supersônico. É importante ressaltar que, nesse teste, não há captura da onda de choque, isto é, a onda de choque observada nas figuras subseqüentes desta seção é apenas uma reprodução das condições de contorno do problema.

A figura 5.8 ilustra o domínio computacional, onde a face inferior esquerda contém as condições de contorno que caracterizam a região 1, anterior ao choque, e a face inferior direita contém as condições de contorno que caracterizam a região 2, posterior ao choque. A linha pontilhada representa a linha de choque normal que separa as duas regiões.

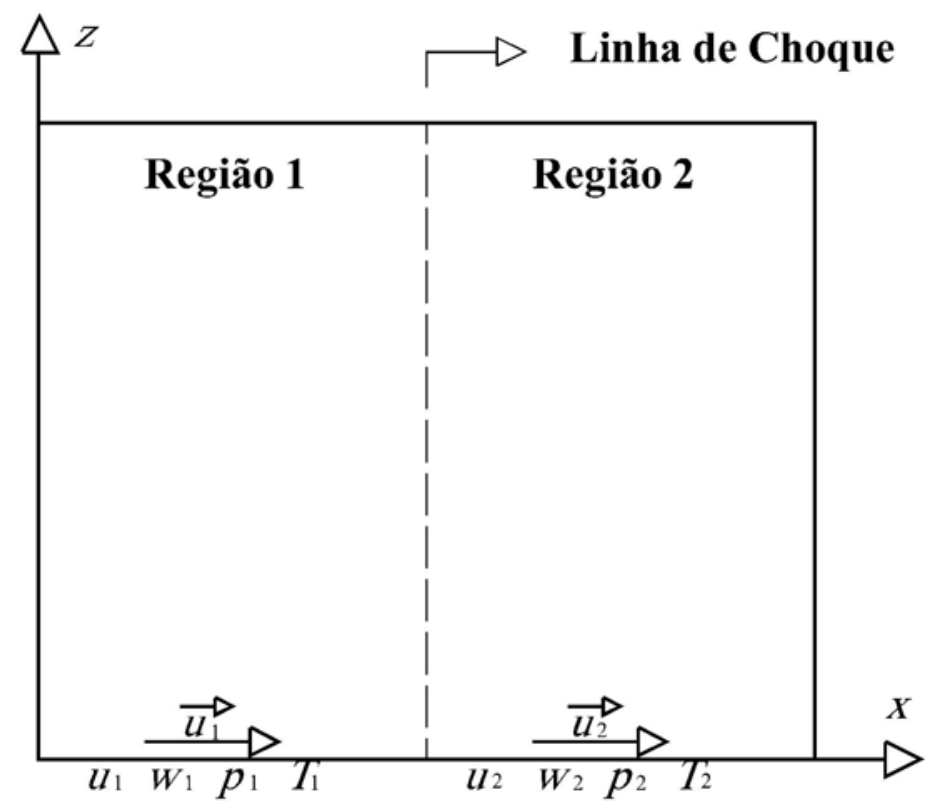

Figura 5.8 - Domínio computacional (1,2 m x 1,2 m) do teste de convergência e estabilidade. 
As condições de contorno da região 1 (anterior ao choque) fornecidas na face inferior esquerda são: temperatura $T_{1}=300 \mathrm{k}$; pressão $p_{1}=1$ bar; velocidade na direção $x$, $u_{1}=694 \mathrm{~m} / \mathrm{s}$ (velocidade normal à linha de choque) e velocidade na direção $z, w_{1}=0$ (velocidade tangencial à linha de choque).

As condições de contorno da região 2 (posterior ao choque) fornecidas na face inferior direita são: temperatura $T_{2}=506,4 \mathrm{k}$; pressão $p_{2}=4,2$ bar; velocidade na direção $x$, $u_{2}=260,2 \mathrm{~m} / \mathrm{s}$ (velocidade normal à linha de choque) e velocidade na direção $z, w_{2}=0$ (velocidade tangencial à linha de choque).

Os valores das condições de contorno de velocidade, pressão e temperatura da região 2 (região posterior ao choque) são obtidas pelas equações de salto, descritas abaixo, segundo Simões-Moreira (2002), para efeito de comparação com os resultados obtidos pelo método numérico desenvolvido neste trabalho.

Portanto, as equações de salto para onda de choque normal são:

- relação entre as pressões das duas regiões

$$
\frac{p_{2}}{p_{1}}=1+\frac{2 \gamma}{\gamma+1}\left(M_{1}^{2}-1\right)
$$

- relação entre as temperaturas das duas regiões

$$
\frac{T_{2}}{T_{1}}=1+\frac{2(\gamma-1)}{(\gamma+1)^{2}} \frac{\gamma M_{1}^{2}+1}{M_{1}^{2}}\left(M_{1}^{2}-1\right) ;
$$

- relação entre as densidades ou velocidades normais das duas regiões

$$
\frac{\rho_{2}}{\rho_{1}}=\frac{u_{1}}{u_{2}}=\frac{(\gamma+1) M_{1}^{2}}{2+(\gamma-1) M_{1}^{2}} .
$$

De acordo com as hipóteses do problema, isto é, as condições de contorno da região 1 (anterior ao choque), o número de Mach da região 1 igual a $\mathrm{M}_{1}=2$; utilizando as relações $u_{1}=M_{1} c_{1}$ e $c_{1}=\sqrt{\gamma R T_{1}}$, onde $c_{1}$ é a velocidade do som na região $1, R$ é a 
constante universal dos gases perfeitos e $\gamma$ é a razão entre os calores específicos do ar e empregando as condições de salto (5.1, 5.2 e 5.3) descritas anteriormente, tem-se as seguintes condições gerais para a região 2 :

- relação entre as pressões

$\frac{p_{2}}{p_{1}}=4,2 \Rightarrow p_{2}=4,2$ bar ;

- relação entre as temperaturas

$\frac{T_{2}}{T_{1}}=1,69 \Rightarrow T_{2}=506,4 \mathrm{~K}$;

- relação entre as velocidades

$\frac{u_{1}}{u_{2}}=2,67 \Rightarrow u_{2}=260,2 \mathrm{~m} / \mathrm{s}$.

Para este teste, com as condições de contorno acima descritas, simulou-se três casos. No primeiro caso, dividiu-se o domínio computacional em 10 células na direção $x$ e 10 células na direção $z$ ( $\Delta x=\Delta z=0,12 \mathrm{~m}$ ), totalizando 100 células na malha computacional. No segundo caso, utilizou-se 20 células em cada direção ( $\Delta x=\Delta z=0,06 \mathrm{~m}$ ) formando uma malha computacional de 400 células e, finalmente, no terceiro caso, utilizou-se 40 células em cada direção ( $\Delta x=\Delta z=0,03 \mathrm{~m}$ ), formando, consequentemente, uma malha computacional de 1600 células. Em todos os três casos, acima descritos, utilizou-se um intervalo de tempo de $10^{-5}$ s e grau 2, na expansão dos polinômios.

As figuras 5.9 à 5.20 mostram o comportamento das velocidades $u$ e $w$, pressão $p$ e temperatura $T$ para os três casos simulados do teste de consistência e estabilidade. 


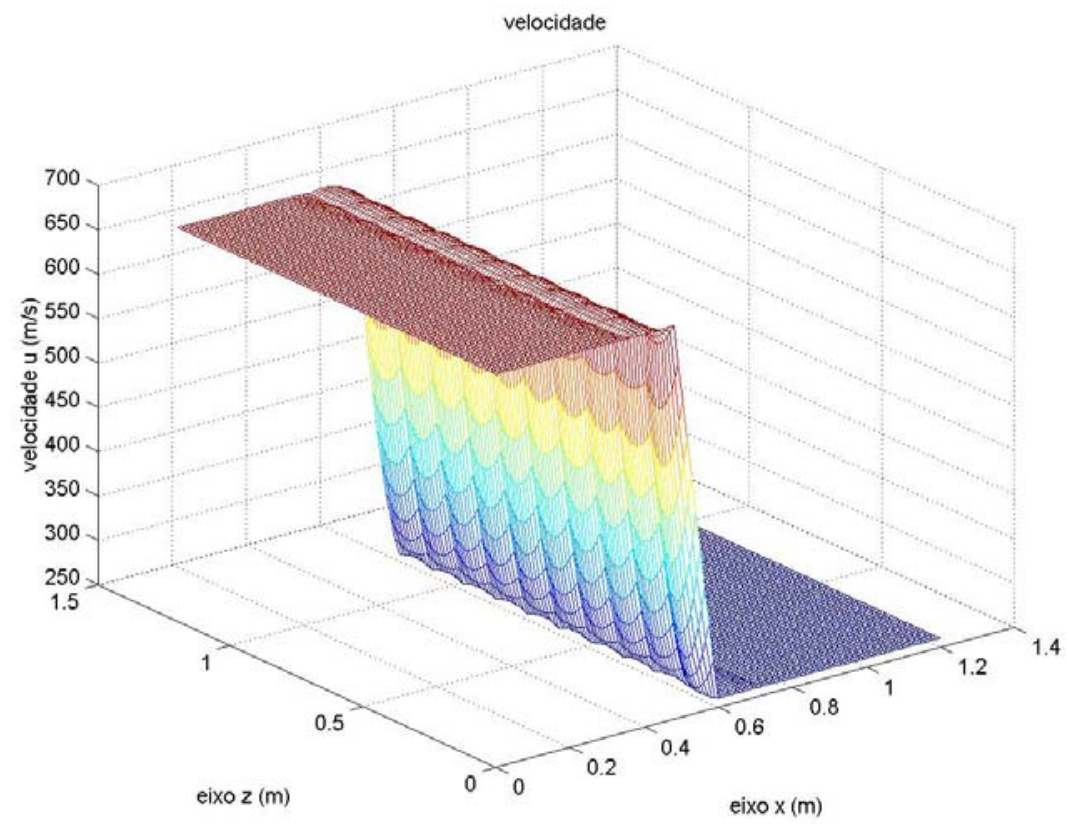

Figura 5.9 - Comportamento da velocidade $u$ (normal ao choque) - malha computacional com 100 células.

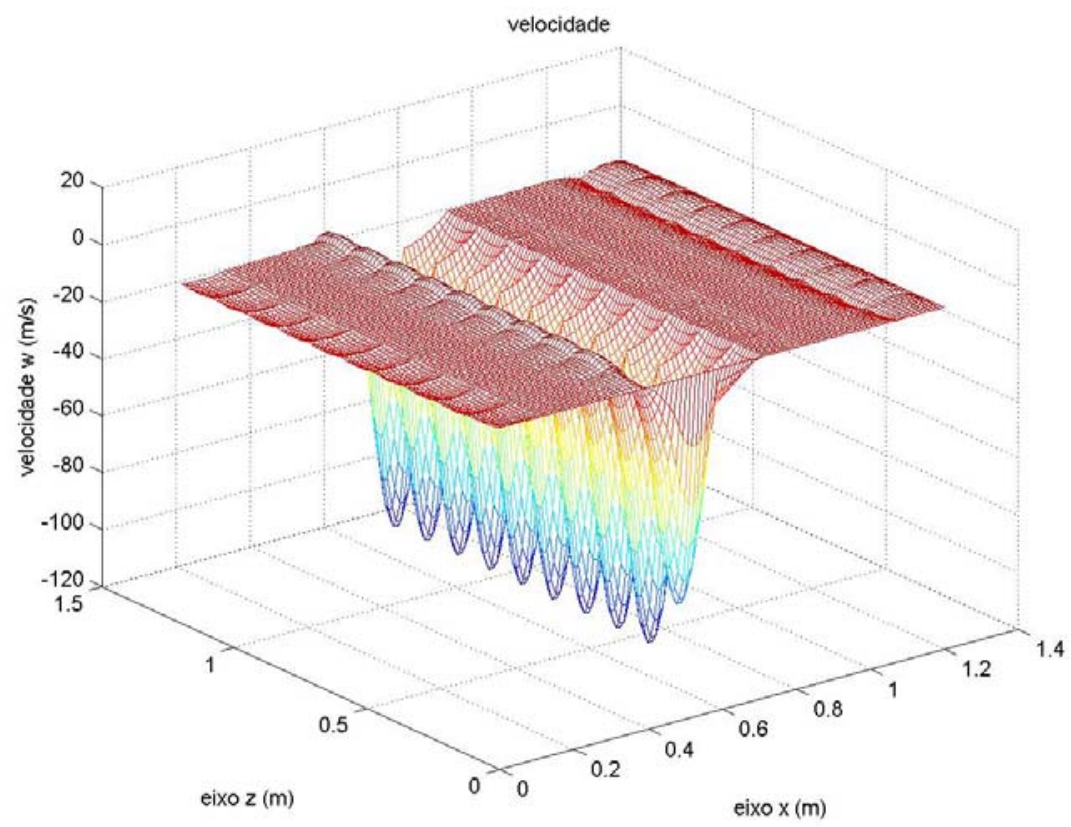

Figura 5.10 - Comportamento da velocidade $w$ (tangencial ao choque) - malha computacional com 100 células. 


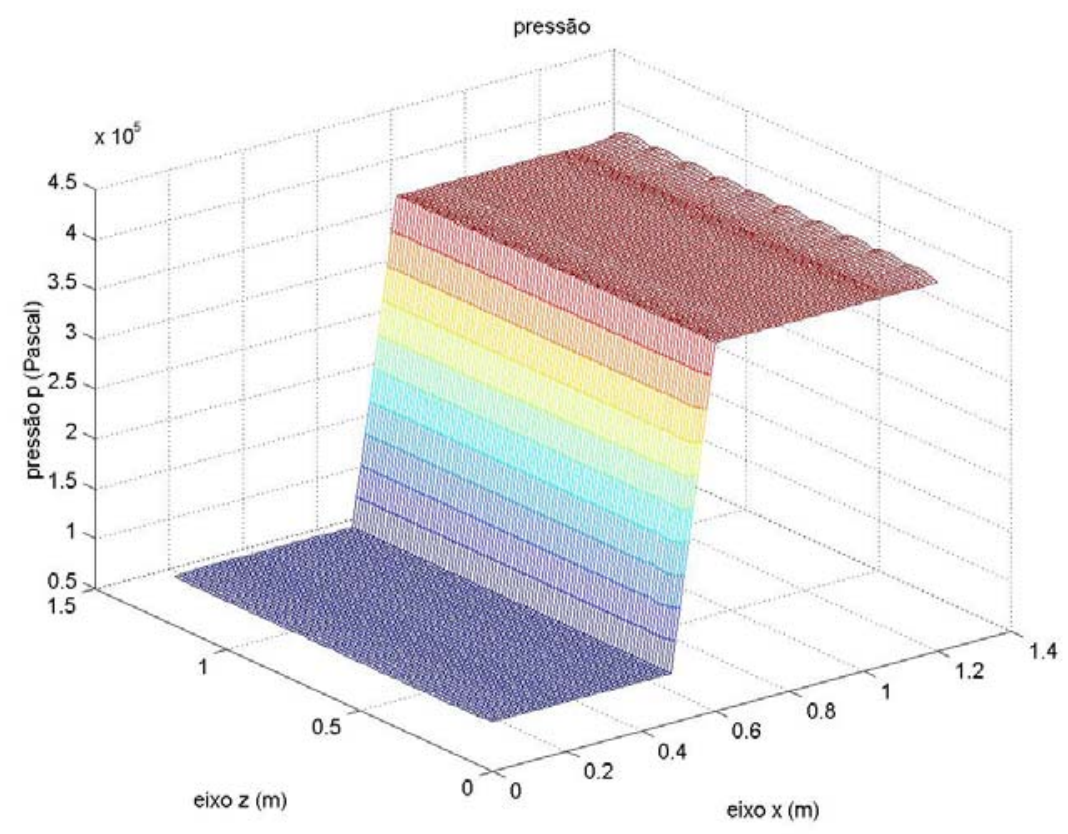

Figura 5.11 - Comportamento da pressão - malha computacional com 100 células.

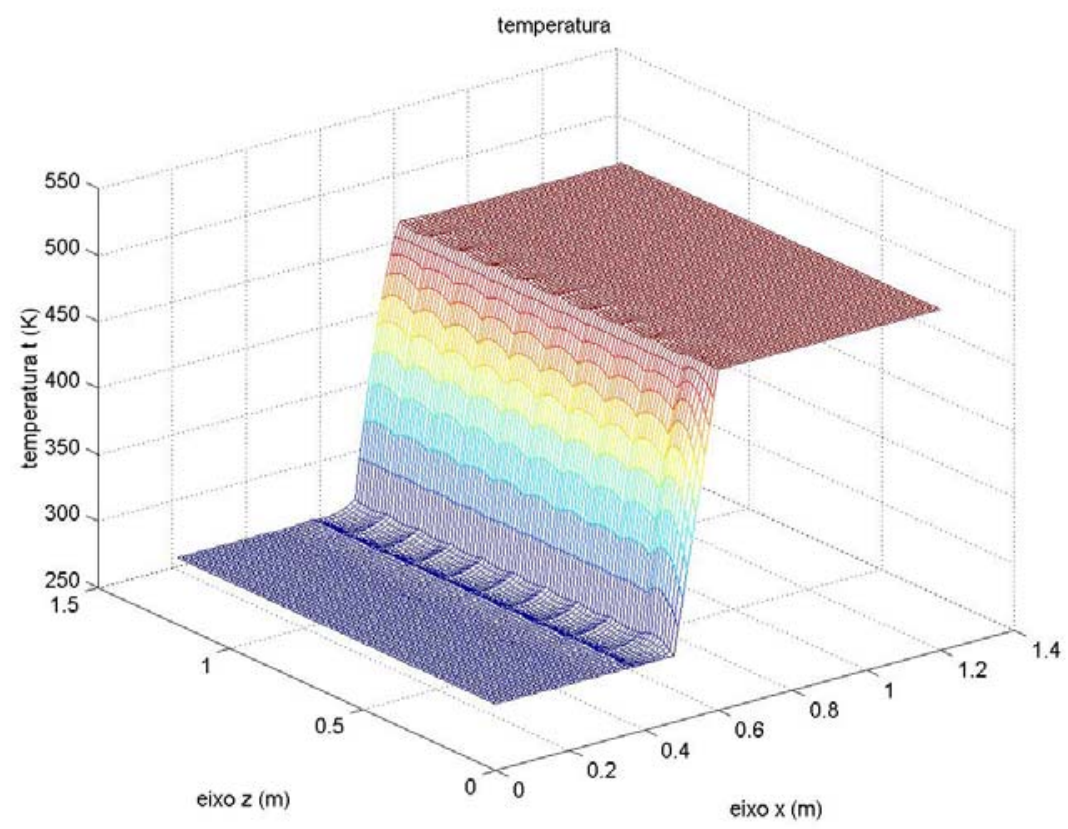

Figura 5.12 - Comportamento da temperatura - malha computacional com 100 células. 


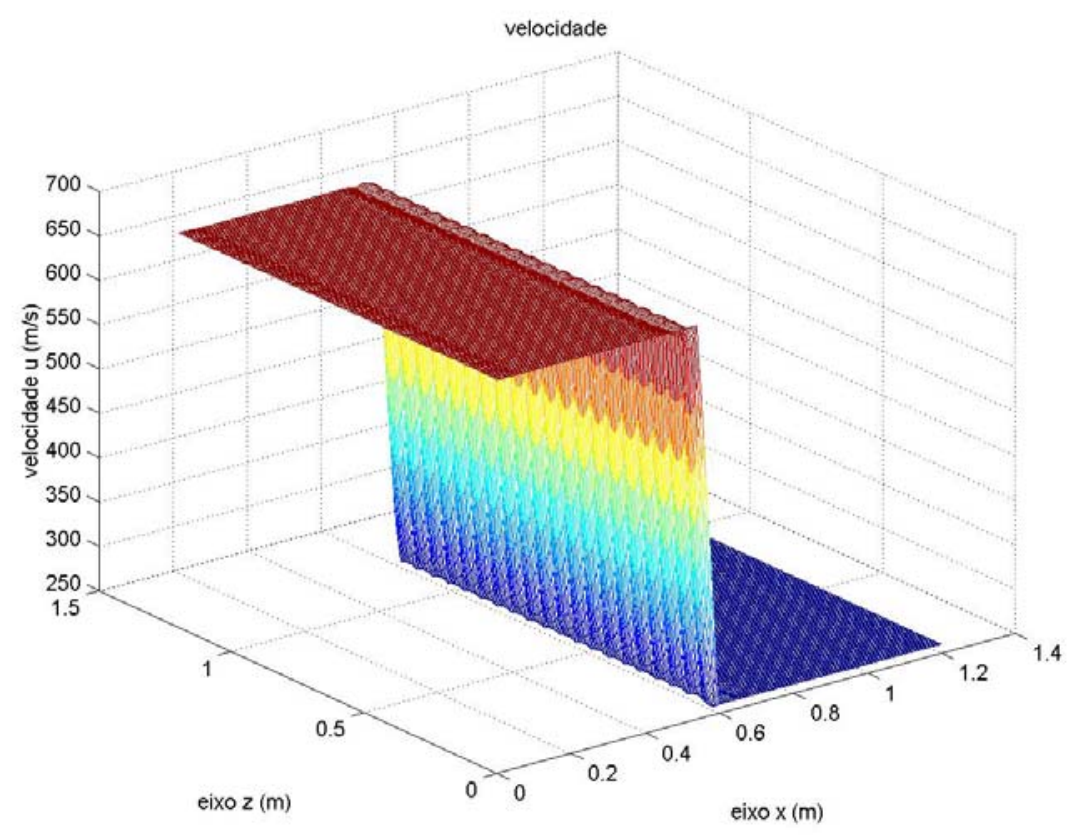

Figura 5.13 - Comportamento da velocidade $u$ (normal ao choque) - malha computacional com 400 células.

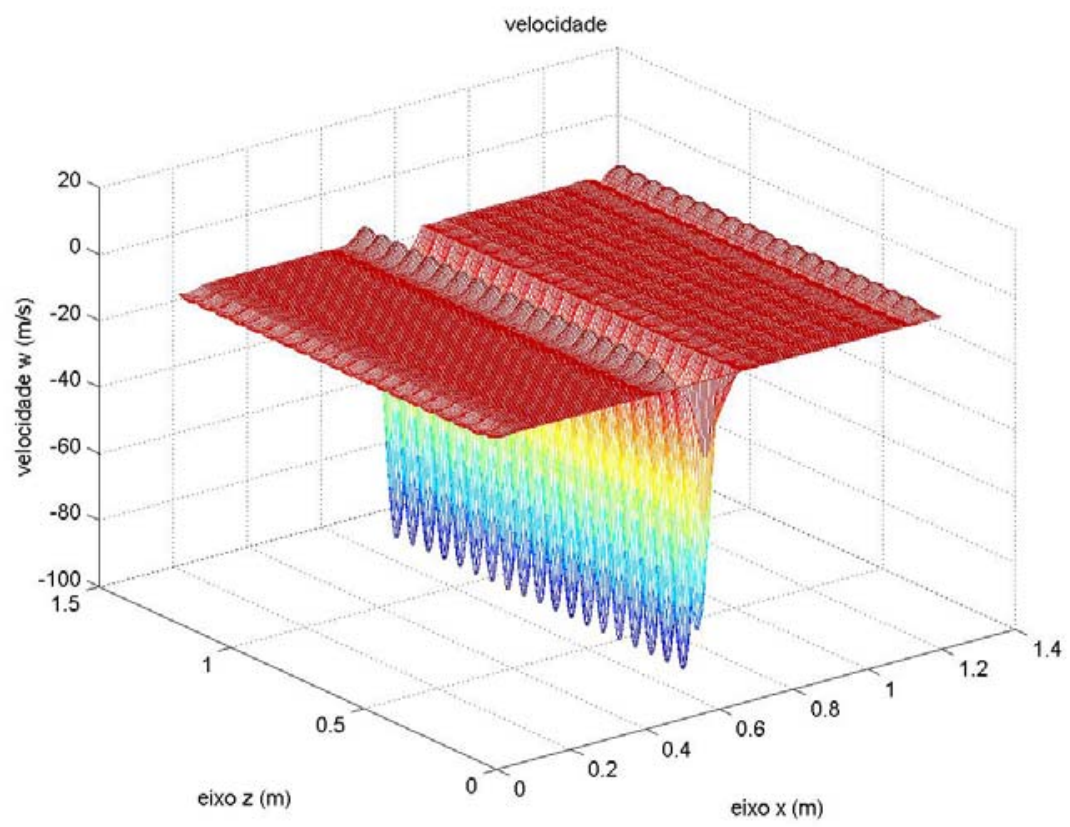

Figura 5.14 - Comportamento da velocidade $w$ (tangencial ao choque) - malha computacional com 400 células. 


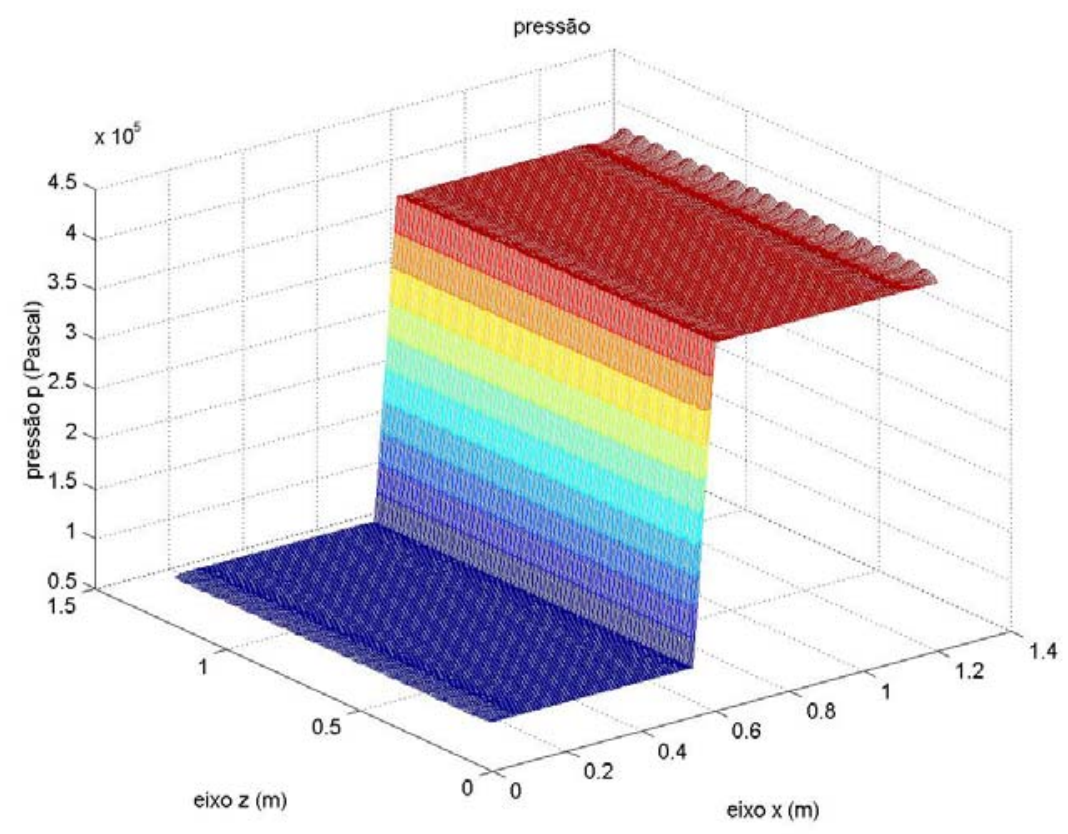

Figura 5.15 - Comportamento da pressão - malha computacional com 400 células.

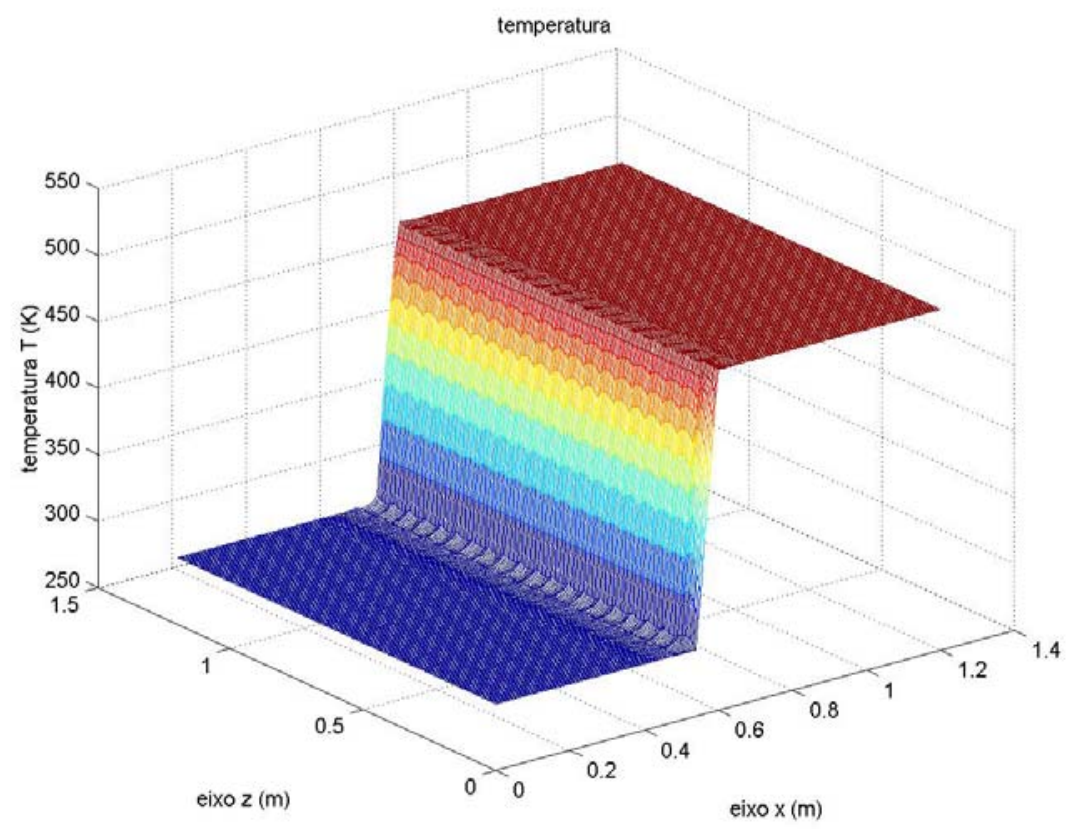

Figura 5.16 - Comportamento da temperatura - malha computacional com 400 células. 


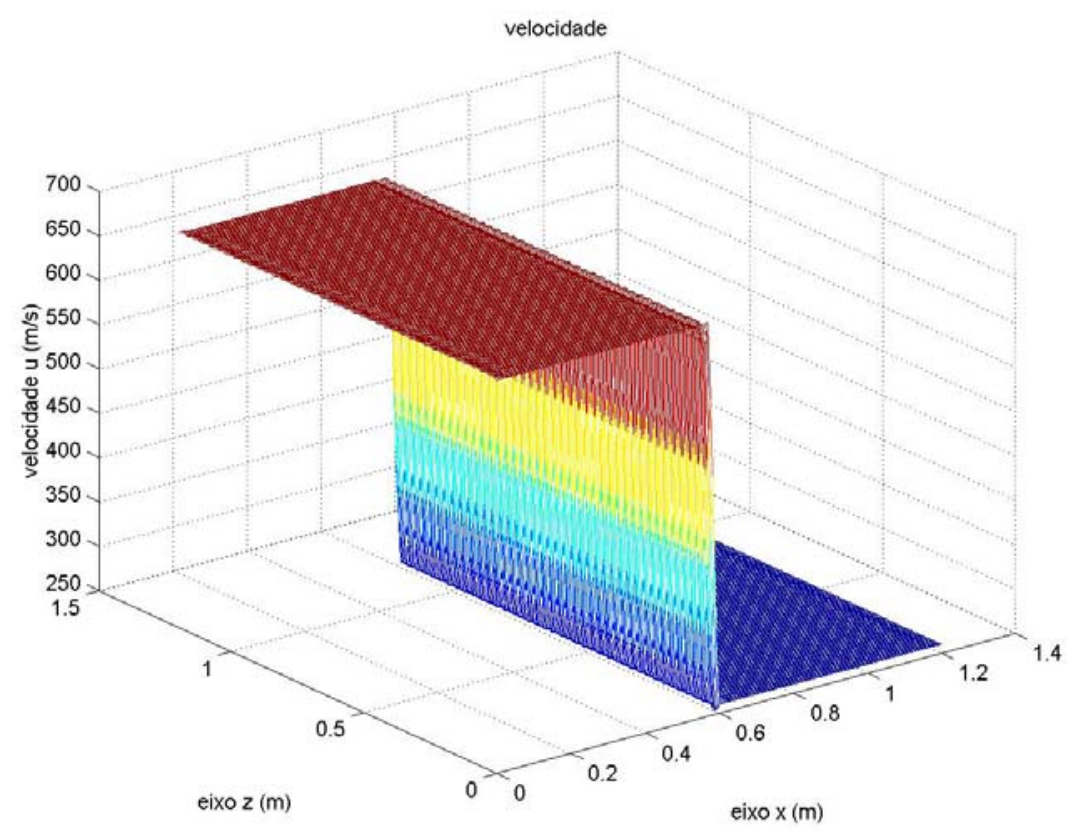

Figura 5.17 - Comportamento da velocidade $u$ (normal ao choque) - malha computacional com 1600 células.

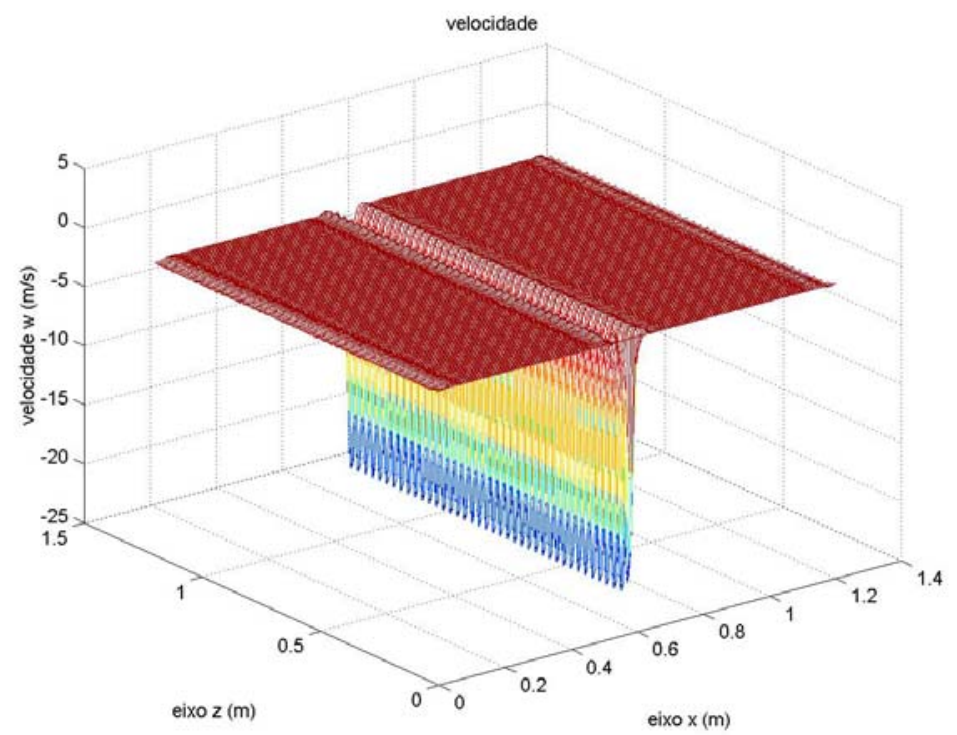

Figura 5.18 - Comportamento da velocidade $w$ (tangencial ao choque) - malha computacional com 1600 células. 


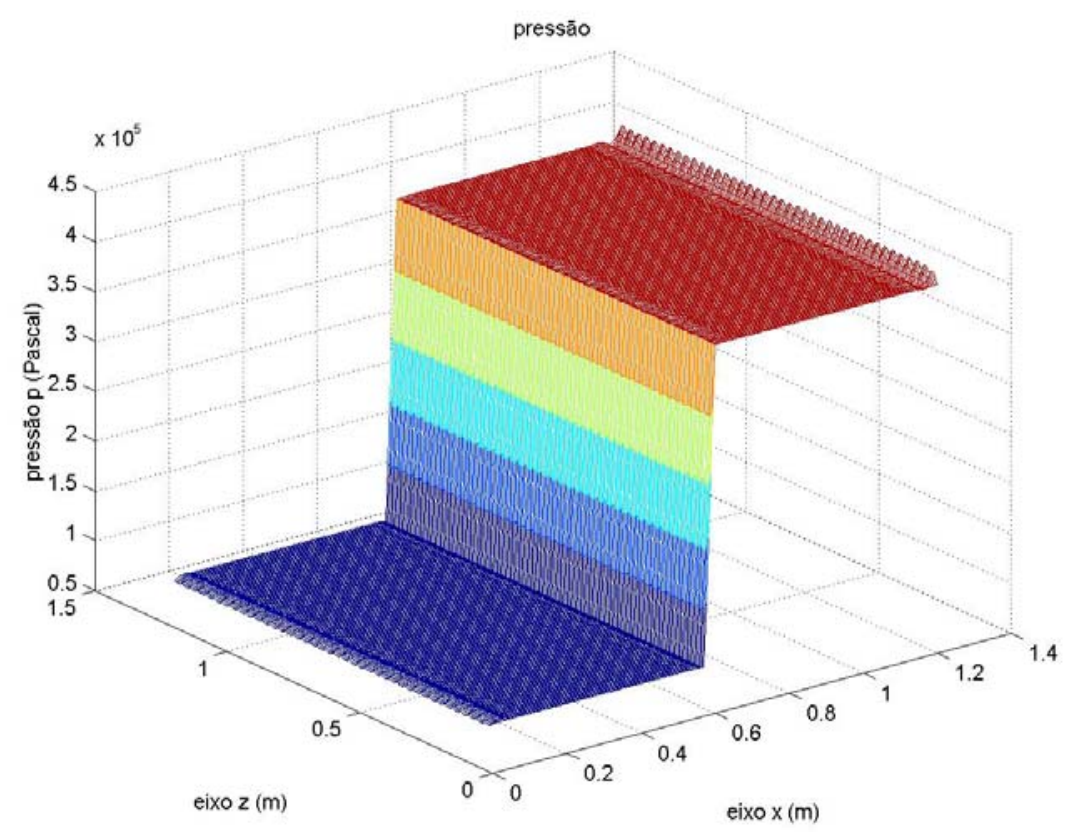

Figura 5.19 - Comportamento da pressão - malha computacional com 1600 células.

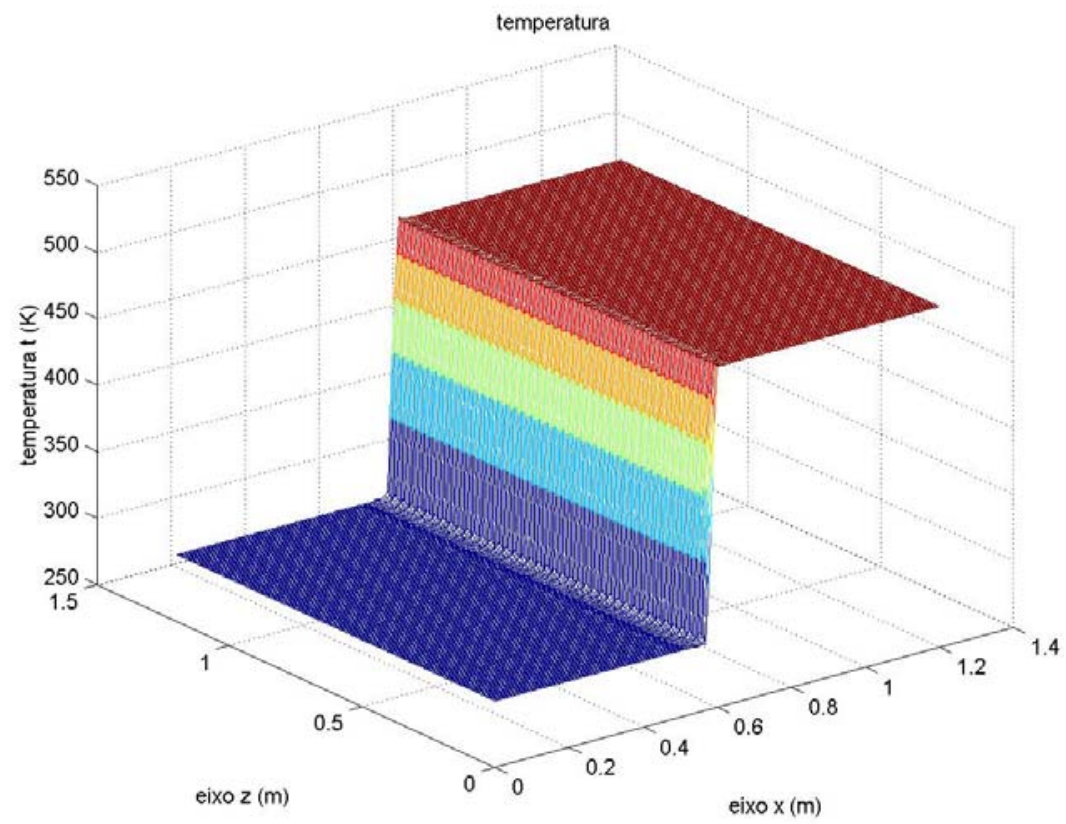

Figura 5.20 - Comportamento da temperatura - malha computacional com 1600 células. 
Nota-se, à medida que a malha computacional utilizada nesses casos fica mais refinada, o comportamento das grandezas físicas analisadas (velocidade $u$ normal à onda de choque, velocidade $w$ tangencial à onda de choque, pressão $p$ e temperatura $T$ ), proveniente das equações discretizadas, tende à solução das equações diferenciais que governam o teste estudado. Por isso que exatamente na interface das duas regiões anterior e posterior ao choque, a velocidade tangencial $w$, que deveria ser zero, oscila em torno desse valor. Para as malhas mais refinadas, essas oscilações foram menores do que para as malhas menos refinadas, isto porque as células menores descreveram melhor geometricamente a interface.

\section{3 - PROBLEMA DO DEGRAU DE TEMPERATURA}

Esse problema simula, numericamente, a interface de descontinuidade de temperatura, de um escoamento supersônico, isto é, simula um degrau de temperatura formado através das condições de contorno impostas pelo problema. O objetivo deste caso é analisar o problema da difusão numérica, ou falsa difusão, que pode ser criada artificialmente pelo método numérico. Esse fato é verificado principalmente em problemas com convecção predominante. A figura 5.21 mostra o domínio computacional do problema.

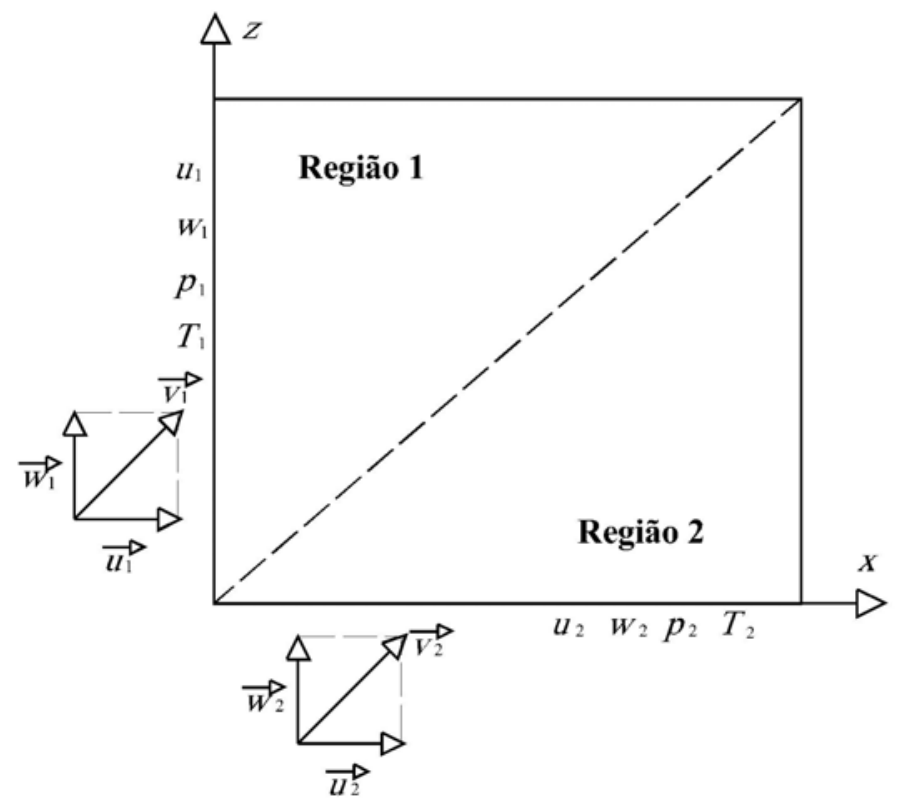

Figura 5.21 - Domínio computacional (2,0 m x 2,0 m) do problema do degrau de temperatura. 
As condições de contorno adotadas para o problema, em questão, estão descritas a seguir. As condições de contorno da região 1 , dadas pela face esquerda do domínio computacional, são: Temperatura $t_{1}=310 \mathrm{~K}$; pressão $p_{1}=1$ bar; velocidade na direção do eixo $x, u_{1}=500 \mathrm{~m} / \mathrm{s}$ e velocidade na direção do eixo $z, w_{1}=500 \mathrm{~m} / \mathrm{s}$. As condições de contorno da região 2, dadas pela face inferior do domínio computacional, são: temperatura $T_{2}=290 \mathrm{~K}$; pressão $p_{2}=1$ bar; velocidade na direção do eixo $x, u_{2}=500 \mathrm{~m} / \mathrm{s}$ e velocidade na direção do eixo $z, w_{2}=500 \mathrm{~m} / \mathrm{s}$. Com a finalidade de gerar o degrau de temperatura, a condição de contorno de temperatura da face esquerda é diferente $20 \mathrm{~K}$ da condição de contorno de temperatura da face inferior.

Para este problema, assume-se as seguintes propriedades para o fluido: fluido invíscito, isto é, $\mu=0$, e condutividade térmica igual a zero, isto é, $\mathrm{k}=0$. Com isto, os termos difusivos da equação da energia são eliminados de forma a não existir nenhum tipo de difusão.

Nessas condições de contorno, as componentes do vetor velocidade do fluido de entrada podem assumir valores distintos. A variação das componentes da velocidade tem por finalidade alterar o ângulo com que o vetor velocidade faz com o eixo $x$ (ângulo $\theta$ ) e, portanto, a direção relativa entre a malha e a velocidade do fluido. $\mathrm{O}$ caso analisado nesta seção tem $\theta=45^{\circ}$.

Para este problema, baseado nas condições de contorno descritas anteriormente, foram desenvolvidos quatro casos, isto é, foram feitas quatro simulações numéricas. Neste problema, em todos os quatro casos estudados, a malha computacional adotada foi de 100 células (10 células na direção $x$ e 10 células na direção $z$ ), com $\Delta \mathrm{x}=\Delta \mathrm{z}=0,2 \mathrm{~m}$ e $\Delta \mathrm{t}=0,00001 \mathrm{~s}$. Os principais dados de cada caso analisado são:

- primeiro caso - malha computacional, acima descrita, e grau 1 na expansão das funções hierárquicas; 
- segundo caso - malha computacional, acima descrita, e grau 2 na expansão das funções hierárquicas;

- terceiro caso - malha computacional, acima descrita, e grau 3 na expansão das funções hierárquicas;

- quarto caso - malha computacional, acima descrita, e grau 4 na expansão das funções hierárquicas.

As figuras 5.22 à 5.25 mostram os resultados dos quatro casos simulados para o degrau de temperatura. Nota-se que para o grau 1 da expansão das variáveis, o degrau de temperatura obtido foi o degrau com canto mais arredondado. À medida que se aumenta o grau de expansão das variáveis, graus 2, 3 e 4, o degrau de temperatura obtido fica com o canto mais reto. Portanto, pode-se dizer que os degraus de temperatura obtidos no segundo, no terceiro e no quarto casos são bastante semelhantes entre si, e se destacam do primeiro caso.

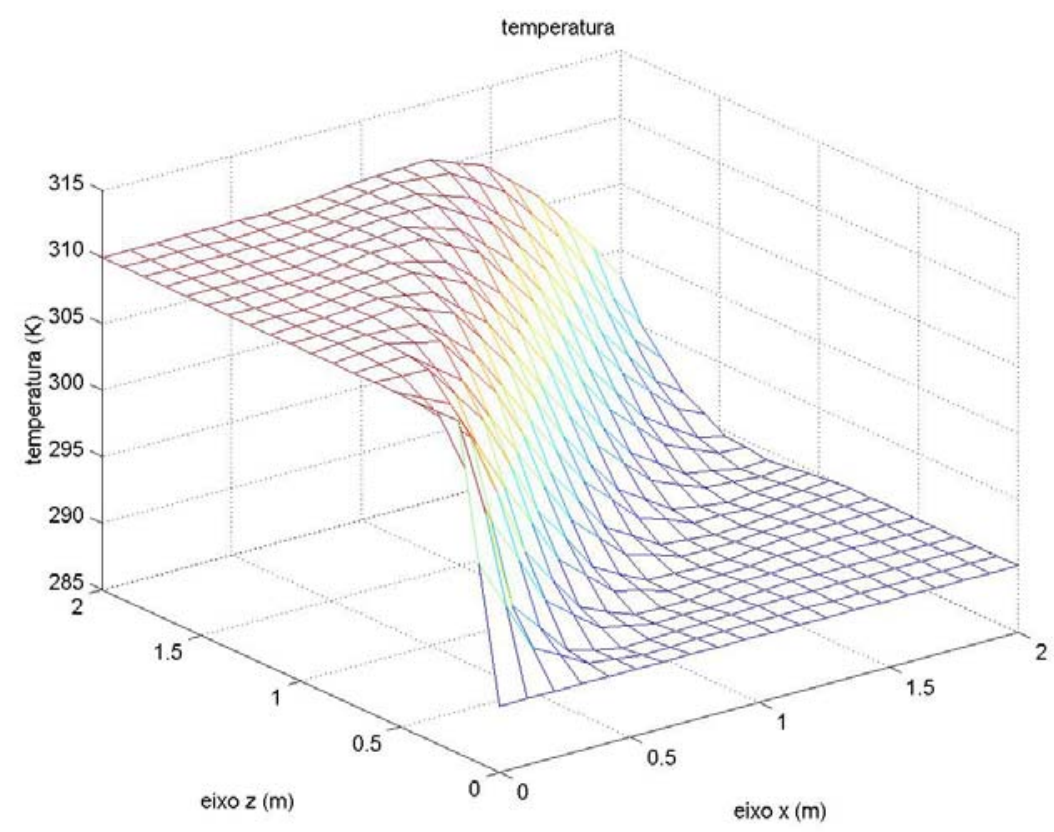

Figura 5.22 - Comportamento da temperatura - malha computacional com 100 células e grau 1 na expansão das variáveis. 


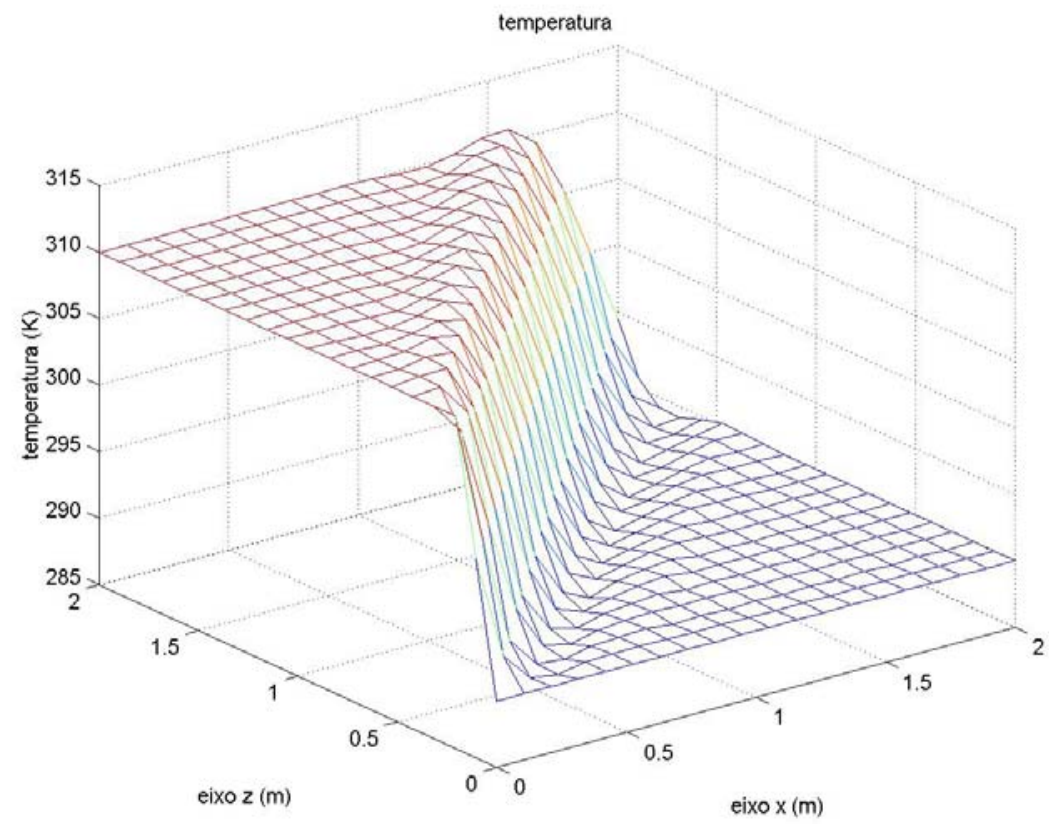

Figura 5.23 - Comportamento da temperatura - malha computacional com 100 células e grau 2 na expansão das variáveis.

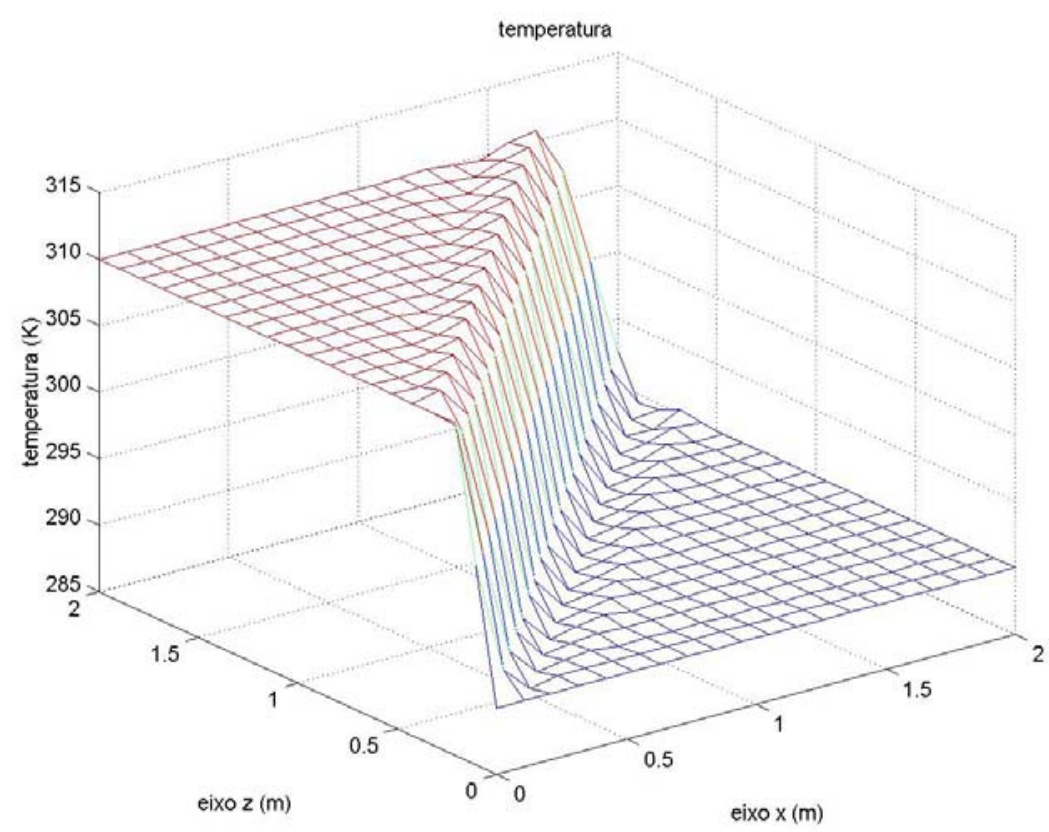

Figura 5.24 - Comportamento da temperatura - malha computacional com 100 células e grau 3 na expansão das variáveis. 


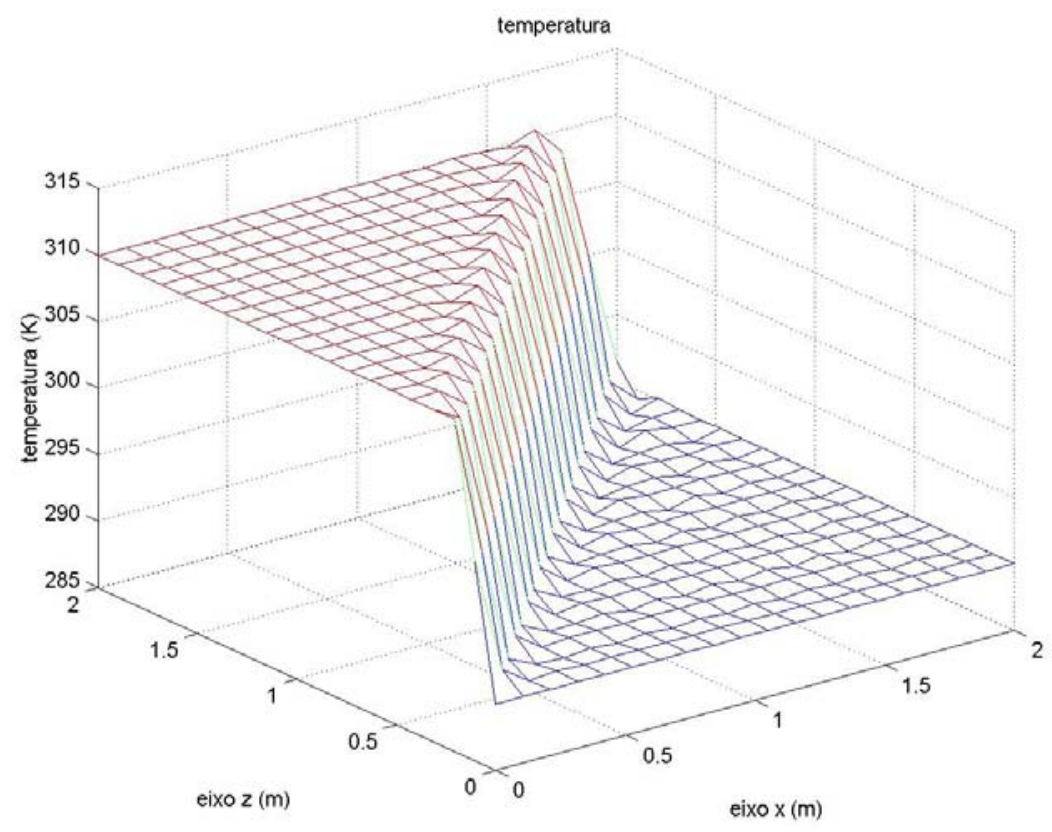

Figura 5.25 - Comportamento da temperatura - malha computacional com 100 células e grau 4 na expansão das variáveis.

\section{4 - PROBLEMA DO CHOQUE OBLÍQUO}

O quarto problema a ser analisado é um escoamento supersônico que incide obliquamente sobre um corpo (superfície), com formação de uma onda de choque oblíqua. Para este tipo de problema, três casos foram desenvolvidos. O primeiro caso visa à captura da onda de choque oblíqua, reta e colada ao corpo, com solução natural (solução de choque fraco). O segundo caso visa à captura da onda de choque oblíqua, reta e colada ao corpo, com solução não natural ou forçada (solução de choque intenso). O terceiro e último caso visa à captura da onda de choque oblíqua, curva e deslocada do corpo. 


\subsection{1 - CHOQUE OBLÍQUO RETO - SOLUÇÃO NATURAL}

Este caso consiste de um escoamento supersônico, com número de Mach da região 1 $\left(M_{1}=2,9\right)$, incidente sobre um corpo (superfície) que ocupa $1 / 3$ do contorno inferior. Nesse caso, com relação ao domínio computacional, mostrado na figura 5.26, o fluido escoa da esquerda para a direita, de cima para baixo, e incide na face esquerda e superior com ângulo $\theta=11,3^{\circ}$, em relação à linha horizontal. A linha pontilhada representa a linha de choque reto que é formada neste escoamento, cujo ângulo de inclinação em relação à direção da velocidade $\overrightarrow{\mathrm{v}}_{1}$ é $\beta=29,4^{\circ}$, Thompson (1972).

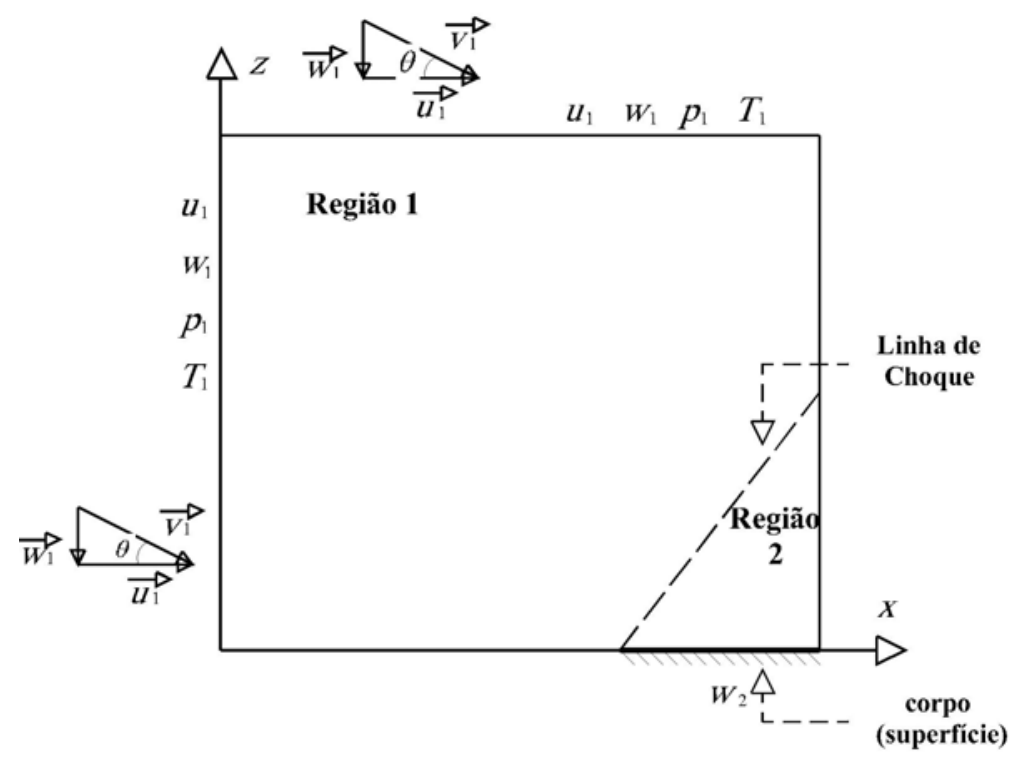

Figura 5.26 - Domínio computacional (1,0 m x 1,0 m) do problema do choque oblíquo reto - solução natural.

As condições de contorno adotadas para esse caso são condições típicas de um escoamento supersônico, para a obtenção da solução natural (solução de choque fraco), isto é, apenas são fornecidas as condições de contorno à montante do escoamento. 
Portanto, as condições de contorno da região 1 (região anterior ao choque), dadas pelas faces esquerda e superior, são: temperatura $T_{1}=300 \mathrm{~K}$; pressão $p_{1}=1$ bar; velocidade na direção $x, u_{1}=1.000,0 \mathrm{~m} / \mathrm{s}$ e velocidade na direção $z, w_{1}=-200,0 \mathrm{~m} / \mathrm{s}$.

A malha computacional empregada nesse caso é composta de 9 células para obtenção da onda de choque, distribuídas igualmente nas direções $x$ e $z$. O comprimento das células nas direções $x$ e $z$, respectivamente, é $\Delta x=\Delta z=0,33 \mathrm{~m}$. O intervalo de tempo utilizado é $\Delta t=10^{-5} \mathrm{~s}$. Quanto à expansão das funções hierárquicas foram usados o grau 1 e o grau 2.

A figura 5.27 mostra as principais grandezas físicas vetoriais das regiões de escoamento 1 e 2, junto à interface de separação das regiões.

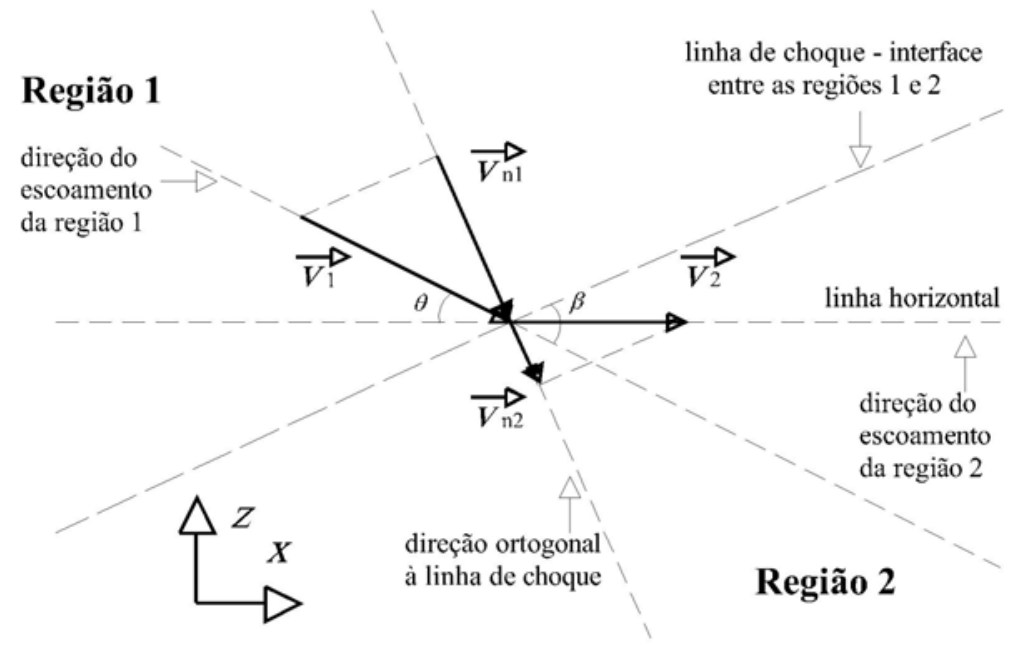

Figura 5.27 - Comportamento da velocidade de escoamento junto à interface de separação das regiões 1 e 2 .

Os resultados obtidos com o método numérico, que mostram a captura da onda de choque para o caso do choque oblíquo - solução natural, podem ser vistos nas figuras 5.28 e 5.29. O gráfico da figura 5.28 mostra a captura da onda de choque utilizando grau 1 na 
expansão das funções, cuja inclinação da onda de choque é de $56^{\circ}$, bem acima do valor esperado da literatura. Já o gráfico da figura 5.29 mostra a captura da onda de choque utilizando grau 2 na expansão das funções, cuja inclinação obtida foi de $36^{\circ}$, valor bem mais próximo dos 29,4 esperado da literatura, Thompson (1972).

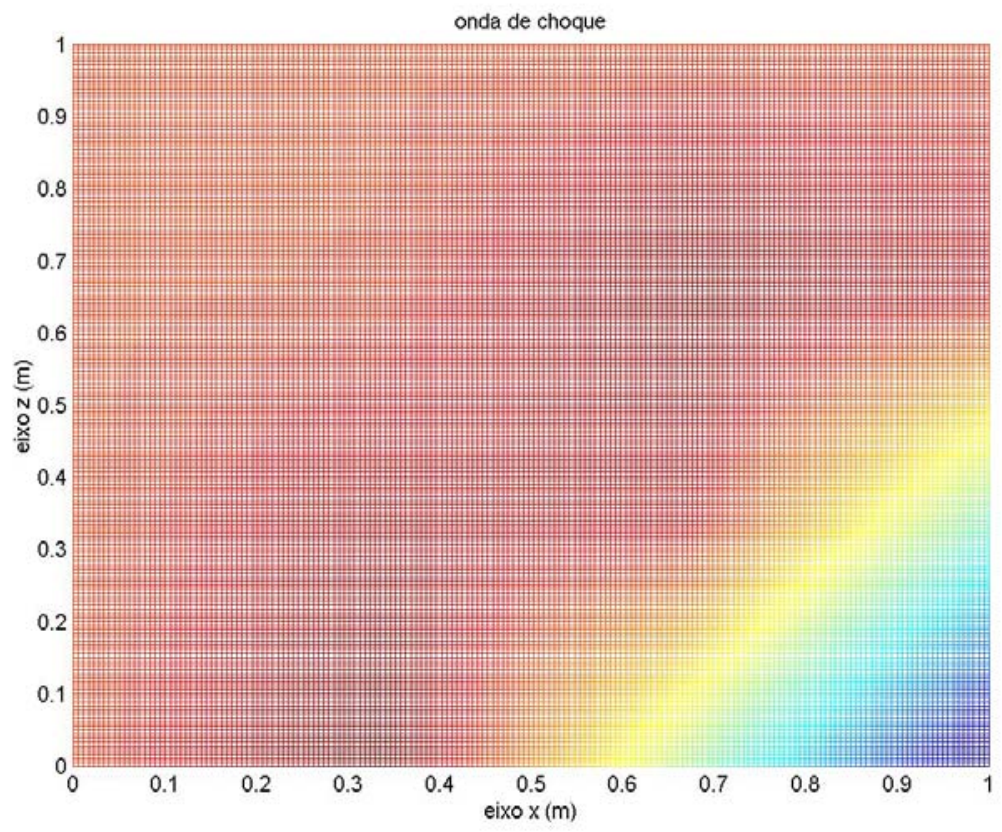

Figura 5.28 - Comportamento da onda de choque - choque oblíquo reto (solução natural), utilizando grau 1 na expansão das variáveis. 


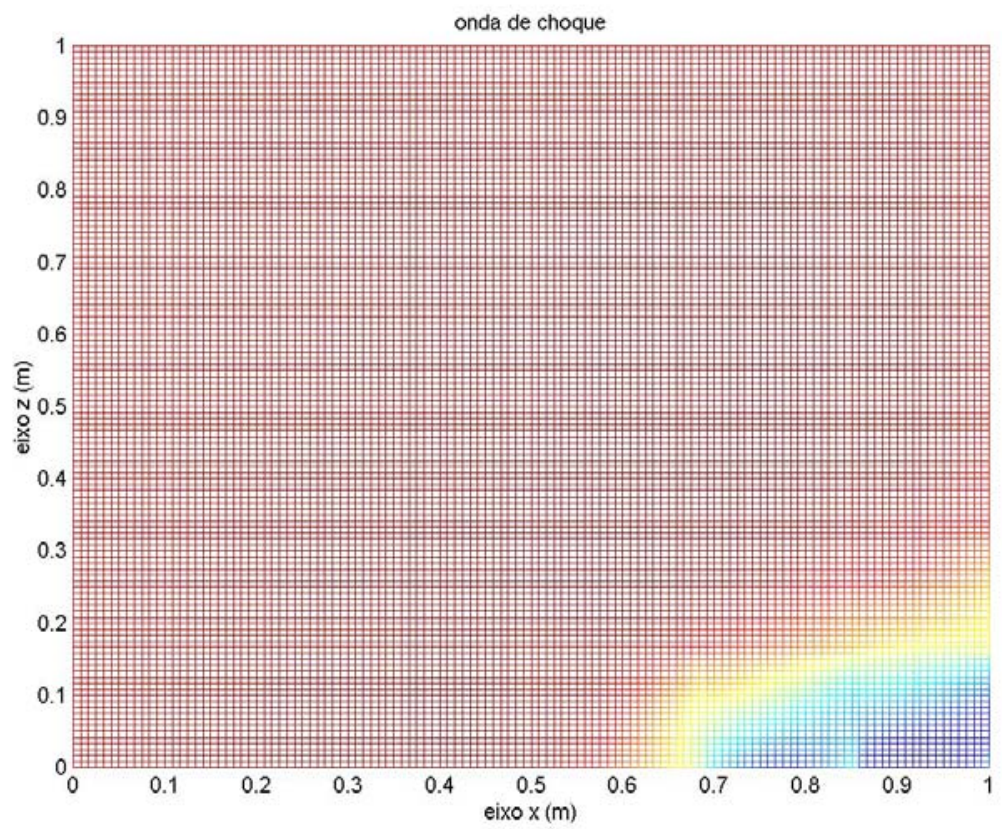

Figura 5.29 - Comportamento da onda de choque - choque oblíquo reto (solução natural), utilizando grau 2 na expansão das variáveis.

\subsection{2 - CHOQUE OBLÍQUO RETO - SOLUÇÃO FORÇADA}

Este caso, assim como o anterior, consiste de um escoamento supersônico, com número de Mach da região $1,\left(\mathrm{M}_{1}=2,9\right)$, incidente sobre um corpo (superfície) que ocupa 1/3 do contorno inferior. A direção do escoamento em relação a este corpo forma um ângulo $\theta=11,3^{\circ}$, figura 5.30 .

Devido à imposição da pressão à jusante do escoamento, região 2, o ângulo de inclinação da onda de choque formada é maior que no caso da solução natural. Portanto, para $M_{1}=2,9$ e $\theta=11,3^{\circ}$, segundo Thompson (1972), (tabela de choque oblíquo, apêndice D), tem-se que $p_{2}=9,6$ bar e $\beta=85^{\circ}$. 


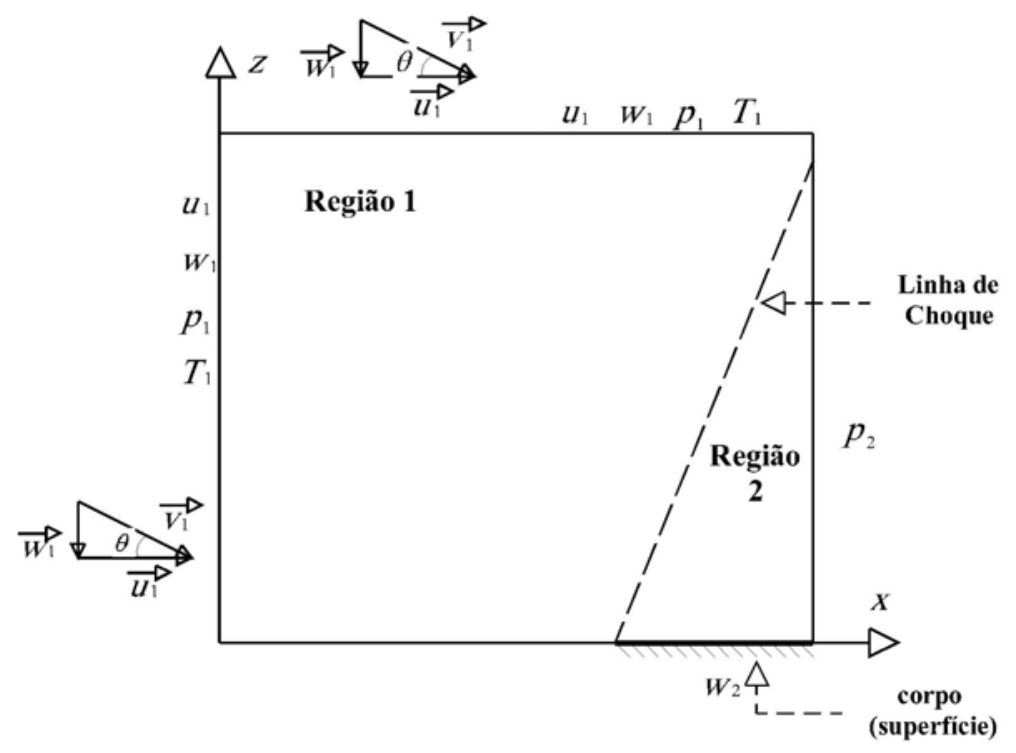

Figura 5.30 - Domínio Computacional (1,0 m x 1,0 m) do problema do choque oblíquo reto - solução forçada.

A malha computacional empregada é a mesma que a do caso anterior, isto é, 9 células com $\Delta x=\Delta z=0,33 \mathrm{~m}, \Delta t=10^{-5} \mathrm{~s}$ e grau 2 na expansão das variáveis.

As condições de contorno adotadas para esse caso são condições típicas de um escoamento supersônico. Para a obtenção da solução forçada (solução de choque intenso), isto é, além das condições de contorno à montante do escoamento (condições de contorno que caracterizam a solução natural) também é fornecida a pressão $p_{2}$ (face esquerda do domínio computacional) da região 2, posterior à linha de choque.

O objetivo deste teste é verificar que, mantendo-se as condições de contorno da região 1 iguais à do caso anterior e adicionando a condição de contorno de pressão $p_{2}$ da região 2 ( $p_{2}=9,6$ bar), obtém-se um ângulo de inclinação $\beta$ da onda de choque formada nesse escoamento igual a $85^{\circ}$, segundo Thompson (1972). Essas condições formam a solução forçada (solução de choque intenso) para o problema do choque oblíquo. 
O resultado obtido, com o método numérico, que mostra a captura da onda de choque para o caso do choque oblíquo - solução forçada, pode ser visto na figura 5.31. O gráfico dessa figura mostra a captura da onda de choque utilizando grau 2 na expansão das funções, cuja inclinação da onda de choque é de $79,5^{\circ}$, comparado com os $85^{\circ}$ valor esperado da literatura, Thompson (1972).

Nota-se que a parte superior da onda de choque encurva ao se aproximar do contorno superior. Isso ocorre devido ao fato desse contorno não possuir a condição de pressão à jusante do escoamento, como descrito acima, que caracteriza a solução forçada da obtenção da onda de choque oblíquo reto.

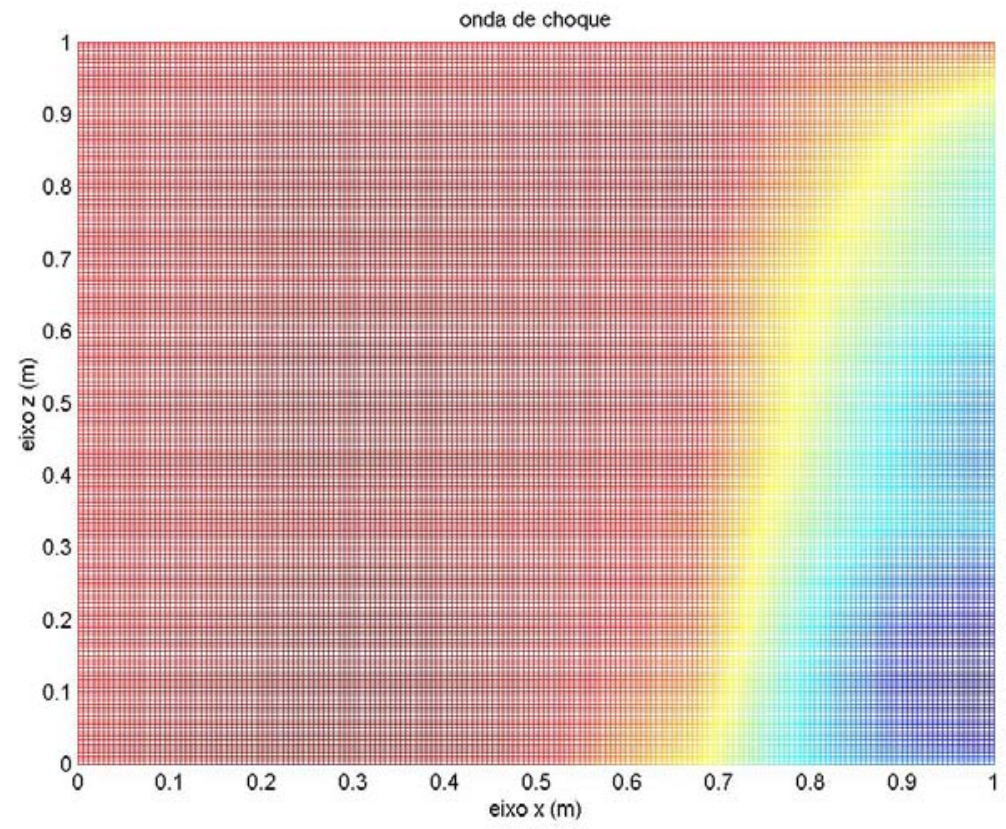

Figura 5.31 - Comportamento da onda de choque - choque oblíquo reto (solução forçada), utilizando grau 2 na expansão das variáveis. 


\subsection{3 - CHOQUE OBLÍQUO CURVO}

Este caso consiste de um escoamento supersônico, com número de Mach da região 1 , $\left(M_{1}=2,9\right)$, incidente sobre um corpo (superfície) que ocupa $1 / 3$ do contorno inferior. Nesse caso, porém, a direção do escoamento em relação a este corpo forma um ângulo $\theta=45^{\circ}$, figura 5.32 .

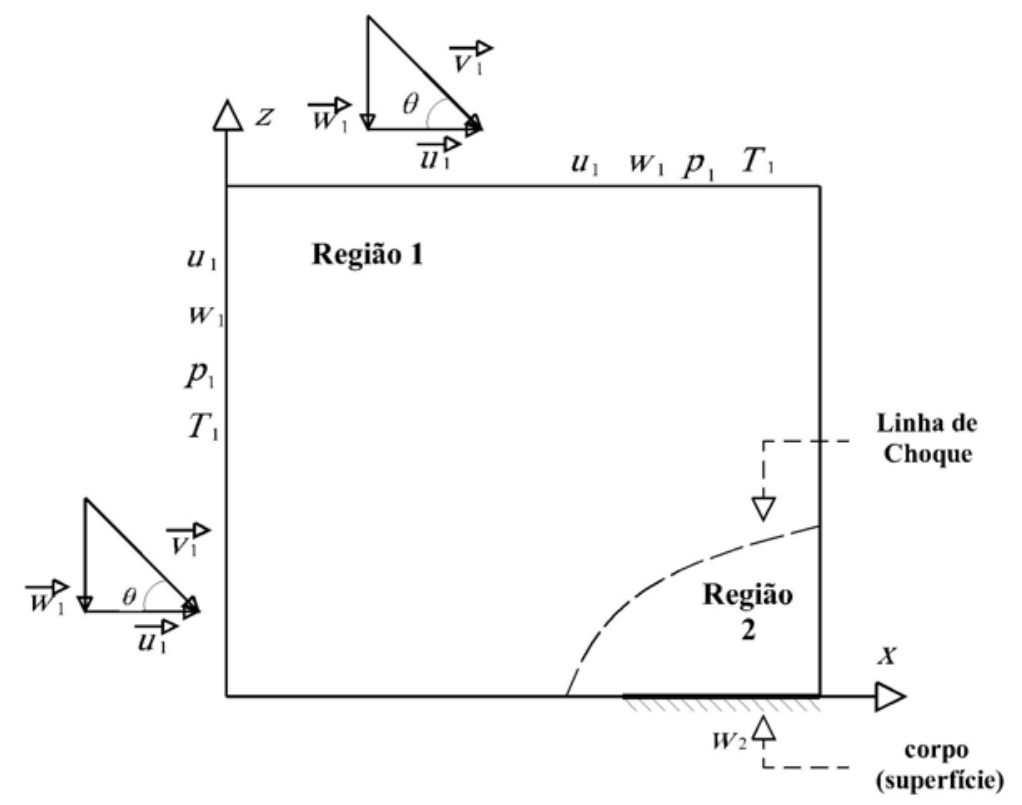

Figura 5.32 - Domínio Computacional (1,0 m x 1,0 m) do problema do choque oblíquo curvo.

Em razão do ângulo de inclinação $\theta$, usado neste caso, ser maior que o ângulo de inclinação máximo ( $\theta$ max $=33,4^{\circ}$ ) e para o escoamento com número de Mach da região 1 $\left(M_{1}=2,9\right)$, tem-se que a onda de choque formada é curva e deslocada do corpo (superfície), Thompson (1972).

A malha computacional utilizada nesse caso é a mesma que a utilizada nos dois casos anteriores, isto é, 9 células com $\Delta x=\Delta z=0,33 \mathrm{~m}, \Delta t=10^{-5} \mathrm{~s}$, porém com grau 3 na expansão das variáveis. 
O objetivo desse teste é verificar que, mantendo-se as condições de contorno da região 1 iguais as dos dois casos anteriores, porém com $u=700 \mathrm{~m} / \mathrm{s}$ e $w=700 \mathrm{~m} / \mathrm{s}$ de tal forma a manter o número de Mach da região 1, obtém-se uma onda de choque oblíqua, curva e deslocada do corpo (superfície), isto é, para esse caso não existe solução para onda de choque oblíqua reta.

A figura 5.33 mostra o resultado obtido com o método numérico para este caso. Nota-se que mesmo mantendo o número de Mach da região 1 , $\left(\mathrm{M}_{1}=2,9\right)$, porém aumentando a inclinação do escoamento em relação à superfície para $45^{\circ}$, de tal forma que o ângulo de inclinação, para este número de Mach, seja maior que o ângulo máximo $\left(33,3^{\circ}\right)$, a onda de choque oblíqua torna-se curva e deslocada da ponta da superfície.

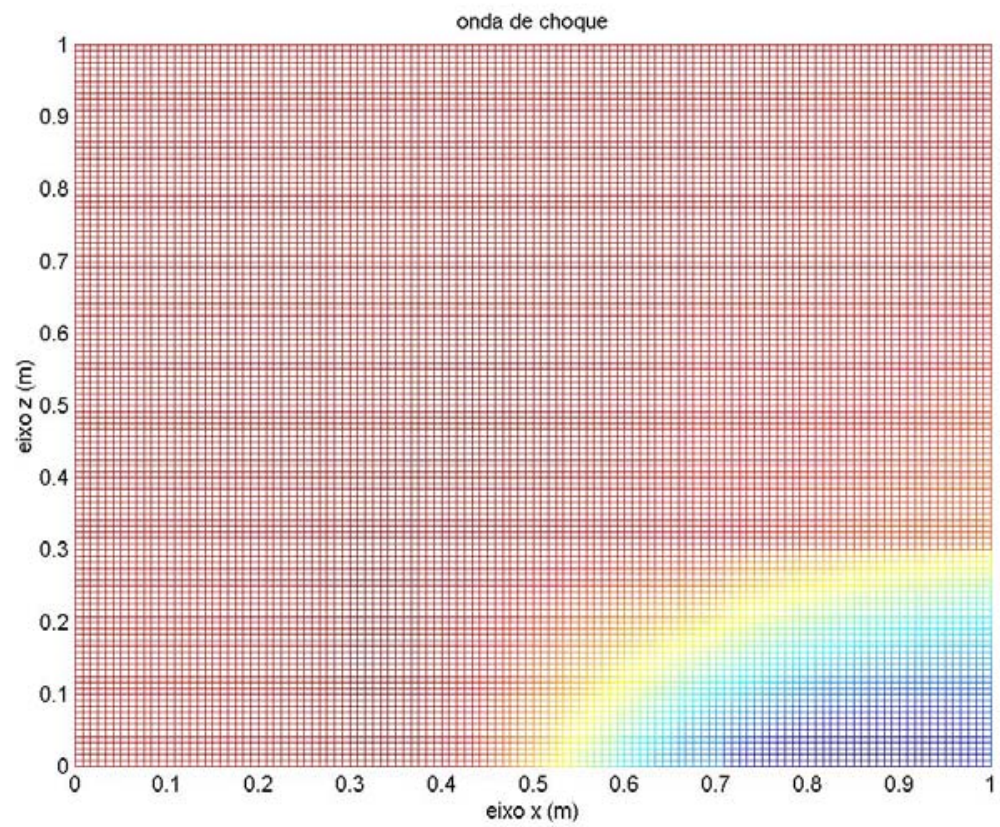

Figura 5.33 - Comportamento da onda de choque - choque oblíquo curvo e deslocado do corpo, utilizando grau 3 na expansão das variáveis. 


\section{6 - CONCLUSÕES}

Lembrando que o objetivo deste trabalho é desenvolver um método numérico, baseado no método dos elementos finitos, que utiliza a formulação Petrov-Galerkin e que emprega, na expansão das variáveis, o método da expansão em funções hierárquicas, para a solução das equações de Navier-Stokes em duas dimensões, para fluidos compressíveis em alta velocidade.

Para alcançar esse objetivo e, consequentemente, verificar o desempenho do método numérico desenvolvido neste trabalho, foi elaborado um programa computacional denominado PMEFH2D. Com este programa foram realizados vários testes.

Antes de se analisar qualquer aspecto do método numérico, verificou-se a reprodução das condições de contorno através de um teste simples de longa duração, conforme descrito na seção 5.1. Com esse teste, verificou-se que o programa não gerava nenhum tipo de perturbação indesejada, na solução do sistema de equações diferenciais desenvolvido no Capítulo 4.

Um segundo teste foi elaborado, no qual verificou-se a consistência e a estabilidade do método numérico desenvolvido, através da simulação de um choque normal, imposto através das condições de contorno do problema, isto é, sem captura da onda de choque, conforme descrito na seção 5.2. Com esse teste verificou-se, de acordo com os resultados obtidos, que a aproximação numérica reproduziu as equações diferenciais na medida em que, mantendo-se o grau de expansão das variáveis, refinou-se a malha computacional. Com esse resultado, pode-se dizer que o método numérico desenvolvido é consistente e estável, isto é, que o método é convergente. 
Um terceiro teste foi realizado para verificar o comportamento do método numérico quanto à dissipação numérica. Para isso, simulou-se um degrau de temperatura, conforme descrito na seção 5.3. Com esse teste verificou-se que para uma expansão das variáveis, de grau 2 em diante, mesmo usando malha não refinada, o método não apresenta dissipação numérica. Para uma expansão das variáveis, de grau 1, uma malha mais refinada é necessária para evitar esse tipo de problema.

\section{1 - CONCLUSÃO}

Simulações de escoamentos supersônicos, incidentes obliquamente sobre um corpo, foram realizadas para verificar a capacidade do método numérico desenvolvido em simular, adequadamente, escoamentos de fluidos compressíveis em alta velocidade e de capturar ondas de choque.

Três casos foram simulados para se observar a captura da onda de choque: choque oblíquo reto com solução natural colado à superfície (solução de choque fraco); choque oblíquo reto com solução forçada colado à superfície (solução de choque intenso) e choque oblíquo curvo descolado da superfície.

Para os três casos analisados, verificou-se, através dos resultados obtidos com o método numérico desenvolvido neste trabalho, uma correspondência muito grande do comportamento das variáveis calculadas com os resultados teóricos. De um modo geral, o erro das variáveis calculadas foi pequeno, quando comparadas com os valores previstos pela literatura.

Através da análise feita, em função dos resultados obtidos nas simulações realizadas, pode-se concluir que o objetivo deste trabalho foi alcançado de maneira satisfatória, pois os resultados obtidos com o método desenvolvido neste trabalho foram qualitativamente e quantitativamente bons, quando comparados com os resultados teóricos encontrados na literatura. 


\section{2 - TRABALHOS FUTUROS}

Em função dos resultados obtidos, pode-se sugerir os seguintes estudos como trabalhos futuros, para que o método numérico torne-se mais abrangente, versátil e eficaz: estender o método numérico, aqui desenvolvido, para poder ser aplicado em malhas irregulares, e com isso poder simular escoamentos supersônicos de fluidos através de objetos das mais diversas geometrias; desenvolver e implantar no programa computacional a capacidade de ajustar o grau do polinômio durante a solução do problema, e com isso possibilitar um avanço muito grande do ponto de vista operacional; e, por último, estender o método numérico para ser aplicado em escoamentos supersônicos de fluidos em três dimensões. 


\section{ANEXO A: FUNÇÕES DE EXPANSÃO HIERÁRQUICAS UTILIZADAS NO PROGRAMA COMPUTACIONAL DESENVOLVIDO - PMEFH2D}

Como mencionado na seção 4.3, as funções de expansão hierárquicas utilizadas neste trabalho, para a solução das equações de Navier-Stokes, são baseadas em polinômios de Legendre, ajustados nos elementos retangulares de forma conveniente. A associação das funções de expansão aos elementos é feita de forma a definir funções de canto, de lado e de área. A ordem das funções associadas aos lados e às áreas dos elementos é definida de forma a ter o grau necessário ou desejado. Neste trabalho as funções de expansão estão preparadas para utilizar grau 1 até 6. O método de quadratura de Gauss, segundo Press et al. (1986), é utilizado para o cálculo das integrais envolvidas na solução das equações de conservação, descritas na seção 4.2. Ressalta-se que, embora o grau das funções de expansão adotadas neste trabalho esteja limitado até 6 , nada impede que sejam acrescidos graus maiores, para a solução de problemas que exijam um maior detalhamento.

A Figura (A.1) apresenta um elemento típico e os parâmetros hierárquicos associados com o seus cantos, lados e áreas.

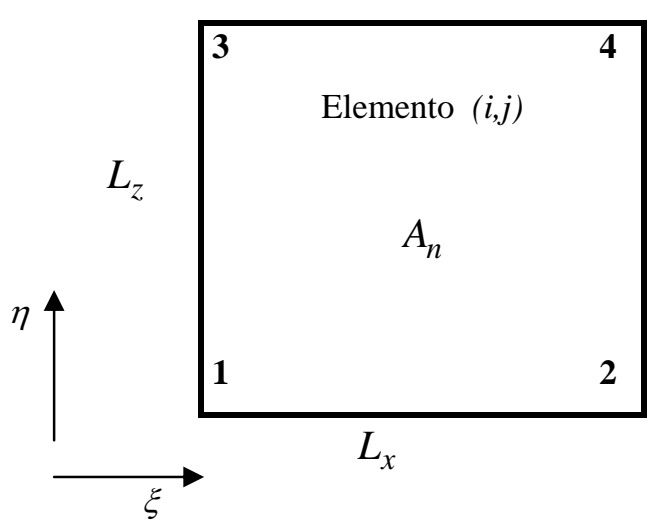

Figura A.1 - Elemento retangular de duas dimensões e seus parâmetros associados. 
Como visto no item 4.3, os polinômios de Legendre, de grau 2 a 6, utilizados neste trabalho, indicados por $L_{g},(\xi)$, onde o índice $g$ indica o grau do polinômio e $\xi$ é a variável local do elemento que varia de -1 a 1 , são os seguintes:

$$
\begin{aligned}
& L_{2}(\xi)=-\left(\xi^{2}-1\right) ; \\
& L_{3}(\xi)=2\left(\xi^{3}-\xi\right) ; \\
& L_{4}(\xi)=\frac{1}{4}\left(15 \xi^{4}-18 \xi^{2}+3\right) ; \\
& L_{5}(\xi)=\left(7 \xi^{5}-10 \xi^{3}+3 \xi\right) ; \mathrm{e} \\
& L_{6}(\xi)=\frac{1}{4}\left(105 \xi^{6}-175 \xi^{4}+75 \xi^{2}-5\right) .
\end{aligned}
$$

Para a direção $\eta$, os polinômios adotados são idênticos aos da direção $\xi$, substituindo-se, simplesmente, $\xi$ por $\eta$ nas equações anteriores e são indicados por $L_{g}(\eta)$.

Para formar as funções de expansão são utilizadas, além dos polinômios de Legendre, funções lineares de acordo com o apresentado no item 4.3. Essas funções lineares formam as funções de expansão associadas aos cantos, da seguinte forma:

\section{Cantos:}

$$
\begin{aligned}
& N_{C 1}=\frac{1}{4}(\xi-1)(\eta-1) ; \\
& N_{C 2}=-\frac{1}{4}(\xi+1)(\eta-1) ; \\
& N_{C 3}=-\frac{1}{4}(\xi-1)(\eta+1) ;
\end{aligned}
$$




$$
N_{C 4}=\frac{1}{4}(\xi+1)(\eta+1)
$$

Nessas funções, o subscrito $c$ indica canto e o número após o subscrito indica o canto ao qual a função está associada.

As funções de expansão, associadas aos lados do elemento, são definidas por uma função linear multiplicada por um dos polinômios de Legendre dados pela equações (A-1) à (A-5). Essas funções são indicadas por $N_{L(i-j)}^{g}$, onde o subscrito $L(i-j)$ representa o lado associado à função e o superescrito $g$, o grau do polinômio. Essas funções são as seguintes:

\section{Lado L(1-2):}

$$
\begin{aligned}
& N_{L(1-2)}^{2}=-\frac{1}{2}(\eta-1) L_{2}(\xi) ; \\
& N_{L(1-2)}^{3}=-\frac{1}{2}(\eta-1) L_{3}(\xi) ; \\
& N_{L(1-2)}^{4}=-\frac{1}{2}(\eta-1) L_{4}(\xi) ; \\
& N_{L(1-2)}^{5}=-\frac{1}{2}(\eta-1) L_{5}(\xi) ; \mathrm{e} \\
& N_{L(1-2)}^{6}=-\frac{1}{2}(\eta-1) L_{6}(\xi) .
\end{aligned}
$$




\section{Lado L(3-4):}

$$
\begin{aligned}
& N_{L(3-4)}^{2}=\frac{1}{2}(\eta+1) L_{2}(\xi) ; \\
& N_{L(3-4)}^{3}=\frac{1}{2}(\eta+1) L_{3}(\xi) ; \\
& N_{L(3-4)}^{4}=\frac{1}{2}(\eta+1) L_{4}(\xi) ; \\
& N_{L(3-4)}^{5}=\frac{1}{2}(\eta+1) L_{5}(\xi) ; \mathrm{e} \\
& N_{L(3-4)}^{6}=\frac{1}{2}(\eta+1) L_{6}(\xi) .
\end{aligned}
$$

\section{Lado L(1-3):}

$$
\begin{aligned}
& N_{L(1-3)}^{2}=-\frac{1}{2}(\xi-1) L_{2}(\eta) ; \\
& N_{L(1-3)}^{3}=-\frac{1}{2}(\xi-1) L_{3}(\eta) ; \\
& N_{L(1-3)}^{4}=-\frac{1}{2}(\xi-1) L_{5}(\eta) ; \\
& N_{L(1-3)}^{5}=-\frac{1}{2}(\xi-1) L_{5}(\eta) ; \mathrm{e} \\
& N_{L(1-3)}^{6}=-\frac{1}{2}(\xi-1) L_{6}(\eta) .
\end{aligned}
$$




\section{Lado $L(2-4)$ :}

$$
\begin{aligned}
& N_{L(2-4)}^{2}=\frac{1}{2}(\xi+1) L_{2}(\eta) ; \\
& N_{L(2-4)}^{3}=\frac{1}{2}(\xi+1) L_{3}(\eta) ; \\
& N_{L(2-4)}^{4}=\frac{1}{2}(\xi+1) L_{5}(\eta) ; \\
& N_{L(2-4)}^{5}=\frac{1}{2}(\xi+1) L_{5}(\eta) ; \mathrm{e} \\
& N_{L(2-4)}^{6}=\frac{1}{2}(\xi+1) L_{6}(\eta) .
\end{aligned}
$$

As funções de expansão, associadas com a área do elemento, são formadas pelo produto de dois polinômios de Legendre $L_{g}(\xi)$ e $L_{g}(\eta)$. Essas funções são indicadas por $N_{A}^{g g}$, onde o subscrito A representa a área e o superescrito gg, os graus dos polinômios de Legendre. Essas funções são as seguintes:

Área $A(1-2-3-4)$ :

$$
\begin{aligned}
& N_{A}^{22}=L_{2}(\xi) L_{2}(\eta) ; \\
& N_{A}^{32}=L_{3}(\xi) L_{2}(\eta) ; \\
& N_{A}^{23}=L_{2}(\xi) L_{3}(\eta) ; \\
& N_{A}^{33}=L_{3}(\xi) L_{3}(\eta) ;
\end{aligned}
$$




$$
\begin{aligned}
& N_{A}^{42}=L_{4}(\xi) L_{2}(\eta) ; \\
& N_{A}^{24}=L_{2}(\xi) L_{4}(\eta) ; \\
& N_{A}^{52}=L_{5}(\xi) L_{2}(\eta) ; \\
& N_{A}^{25}=L_{2}(\xi) L_{5}(\eta) ; \\
& N_{A}^{43}=L_{4}(\xi) L_{3}(\eta) ; \\
& N_{A}^{34}=L_{3}(\xi) L_{4}(\eta) ; \\
& N_{A}^{44}=L_{4}(\xi) . L_{4}(\eta) ; \\
& N_{A}^{26}=L_{2}^{35}(\xi) L_{6}(\eta) ; \mathrm{e} \\
& N_{A}^{53}=L_{5}(\xi) L_{3}(\eta) ; \\
& N_{A}^{62}=L_{6}(\xi) L_{2}(\eta) ;
\end{aligned}
$$

Uma vez definidas as funções de expansão, para os cantos, lados e áreas, associam-se determinadas funções para cada grau de expansão solicitado ou desejado. Assim, para expansões de graus 1 a 6 têm-se as seguintes funções associadas:

Grau 1: Somente as funções de canto, equações (A-6) à (A-9).

Grau 2: Funções de canto, equações (A-6) à (A-9);

Funções de Lado $L(1-2)$, equação (A-10); 
Funções de Lado L(3-4), equação (A-15);

Funções de Lado L(1-3), equação (A-20);

Funções de Lado $L(2-4)$, equação (A-25); e

Funções de Área A(1-2-3-4), equação (A-30).

Grau 3: Funções de canto, equações (A-6) à (A-9);

Funções de Lado $L(1-2)$, equações (A-10) e (A-11);

Funções de Lado L(3-4), equações (A-15) e (A-16);

Funções de Lado L(1-3), equações (A-20) e (A-21);

Funções de Lado L(2-4), equações (A-25) e (A-26);

Funções de Área A(1-2-3-4), equações (A-30), (A-31) e (A-32).

Grau 4: Funções de canto, equações (A-6) à (A-9);

Funções de Lado L(1-2), equações (A-10) à (A-12);

Funções de Lado L(3-4), equações (A-15) à (A-17);

Funções de Lado L(1-3), equações (A-20) à (A-22);

Funções de Lado L(2-4), equações (A-25) à (A-27);

Funções de Área A(1-2-3-4), equações (A-30) à (A-35).

Grau 5: Funções de canto, equações (A-6) à (A-9);

Funções de Lado L(1-2), equações (A-10) à (A-13);

Funções de Lado L(3-4), equações (A-15) à (A-18);

Funções de Lado L(1-3), equações (A-20) à (A-23);

Funções de Lado L(2-4), equações (A-25) à (A-28);

Funções de Área A(1-2-3-4), equações (A-30) à (A-39).

Grau 6: Funções de canto, equações (A-6) à (A-9);

Funções de Lado L(1-2), equações (A-10) à (A-14);

Funções de Lado L(3-4), equações (A-15) à (A-19);

Funções de Lado L(1-3), equações (A-20) à (A-24);

Funções de Lado L(2-4), equações (A-25) à (A-29);

Funções de Área A(1-2-3-4), equações (A-30) à (A-44). 


\section{REFERÊNCIAS BIBLIOGRÁFICAS}

AKIN, J. E.; TEZDUYAR, T. E. Calculation of the advective limit of the SUPG stabilization parameter for linear and higher-order elements. Computer Methods in Applied Mechanics and Engineering, v. 193, pp. 1909-1922, 2004.

AliabAdI, S. K.; TEZDUYAR, T. E. Parallel Fluid Dynamics Computations in Aerospace Applications. International Journal for Numerical Methods in Fluids, v. 21, pp. 783-805, 1995.

ALIABADI, S. K.; TEZDUYAR, T. E. Space-Time Finite Element Computation of Compressible Flows Involving Moving Boundaries and Interfaces. Computer Methods in Applied Mechanics and Engineering, v. 107, pp. 209-223, 1993.

ALMEIDA, R. C.; GALEÃO, A. C. An adaptive Petrov-Galerkin for the Compressible Euler and Navier-Stokes Equations. Computer Methods in Applied Mechanics and Engineering, v. 129, pp. 157-176, 1996.

ANDERSON, D. A.; TANNEHILL, J. C. and PLETECHER, R. H. Computational Fluid Mechanics and Heat Transfer. Hemispher Pub. Corp, Washington, USA. 1984.

ARGYRIS, J. H.; DOLTSNIS, J. S. T.; PIMENTA, P. M.; WÜSTENBERG, H. Natural Finite Techniques for Viscous Fluid Motion. Computer Methods in Applied Mechanics and Engineering, v. 45, pp. 3-55, 1984.

BAKER, A. J. Finite Elements in Nonlinear Fluid Dynamics. $2^{\text {nd }}$ Int. Conf. On Computational Methods in Nonlinear Mechanics, The University of Texas, Austin, Texas, pp. 26-30, March, 1979. 
BARSOUM, M. E.; ALEXANDROU, A. N. Stable Finite Element Solutions of Fully Viscous Compressible Flows. Finite Element in Analysis and Design, v. 19, pp. 6987, 1995.

BAUMANN, C. E.; STORTI, M. A.; IDELSHON, S. R. Improving the Convergence rate of the Petrov-Galerkin Techniques for the Solution of Transonic and supersonic flows. International Journal for Numerical Methods in Engineering, v. 34, pp. 543-568, 1992.

BEJAN, A. Convection Heat Transfer, John Wilew \& Sons, 1984.

BORIS, J. P.; BOOK, D. L. Flux Corrected Transport: I SHASTA, A Transport Algorithm that Works. Journal of Computational Physics, v. 11, pp. 38-69, 1973.

BROOKS, A. Ph.D. Thesis, California Institute of Technology, Pasadena, California, 1981.

BROOKS, A.; HUGHES, T. J. Streamline Upwind/Petrov-Galerkin Methods for Advection Dominated Flows. $3^{\text {rd }}$ Int. Conf. On Finite Element Methods in Fluid Flows, Banff, Canada, pp. 283-292, 1980.

BROOKS, A. N.; HUGHES, T. J. R. Streamline Upwind Petrov-Galerkin Formulation for Convection-Dominated Flows with Particular Emphasis on the Incompressible Navier-Stokes Equations. Computer Methods in Applied Mechanics and Engineering, v. 32, pp. 199-259, 1982.

BRUECKNER, F. P.; HEINRICH, J. C. Petrov-Galerkin Finite Element Model for Compressible Flows. International Journal for Numerical Methods in Engineering, v. 32, pp. 255-274, 1991. 
CABRAL, E. L. L. Hierarchical Expansion Method in the Solution of the Two Group Two-Dimensional Neutron Diffusion Equation. Revista Brasileira de Pesquisa e Desenvolvimento, v. 1, no 2, pp. 50-59, 1996.

CHITTUR, S.; VENKATASUBBAN, S. A New Finite Formulation for ALE (Arbitrary Lagrangian Eulerian) Compressible Fluid Mechanics. Int. Eng. Sci. 33, 12, pp. 1743-1762, 1995.

DENDY, J. E. Two Methods of Galerkin Type Achieving Optimum $L_{2}$ Rates of Convergence for First-Order Hyperbolic. SIAM J. Numer. Anal. , 11, pp. 637-653, 1974.

DONEA, J. A Taylor-Galerkin Method for Convective Transport Problems. International Journal for Numerical Methods in Engineering, v. 20, pp. 101-120, 1984.

GHORBANY, M.; SOHEILI, A. R. Moving element free Petrov-Galerkin viscous method. Journal of the Chinese Institute of Engineering, Transactions of the Chinese Institute of Engineers, v. 27, pp. 473-479, 2004.

HU, Z.; ZHA, G. Calculations of 3-D compressible flows using an efficient low diffusion upwind scheme. International Journal for Numerical Methods in Fluids, v. 47, pp. 253-269, 2005.

HUGHES, T. J. R. Recent Progress in the Development and Understanding of SUPG Methods with Special Reference to the Compressible Euler and Navier-Stokes Equations. International Journal for Numerical Methods in Fluids, v. 7, pp. 12611275, 1987.

HUGHES, T. J. T.; BROOKS, A. A Multidimensional Upwind Scheme with no Crosswind Diffusion. Finite Element Methods for Convection Dominated Flows, ASME, New York, 1979. 
HUGHES, T. J. R.; BROOKS, A.N. A Theoretical Framework for Petrov-Galerkin Methods with Discontinuous Weighting Functions: Application to the Streamline Upwind Procedure. Finite Elements in Fluids, v. IV, Wiley, Chichester, 1982.

HUGHES, T. J. R.; TEZDUYAR, T. E. Finite Element Methods for First Order Hyperbolic Systems with Particular Emphasis on the Compressible Euler Equations. Computer Methods in Applied Mechanics and Engineering, 45, pp. 217-284, 1984.

JOHSON, C. Finite Element Methods for Convection-Diffusion Problems. Computer Methods in Engineering and Applied Sciences V, North-Holland, Amsterdam, 1982.

JOHSON, C. Streamline Diffusion Methods for Problems in Fluid Mechanics. Finite Elements in Fluids, v. VI, Wiley, London, pp. 251-261, 1986.

JOHSON, C.; NÄVER, U.; PITKÄRANTA, J. Finite Element Methods for Linear Hyperbolic Problem. Computer Methods in Applied Mechanics and Engineering, v. 45, pp. 285-312, 1984.

JOHSON, C.; SARANEN, J. Streamline Diffusion Methods for Incompressible Euler and Navier-Stokes Equation. Math. Comput. 47, pp. 1-18, 1986.

JOHSON, C.; SZEPESSY, A. A Shock-Capturing Streamline Diffusion Finite Element Method for a Nonlinear Hyperbolic Conservation Law. TECHNICAL REPORT 1986-09, Mathematics Department, Charmers University of Technology, Göteborg, 1986.

JOHSON, C.; SZEPESSY, A. Convergence of a Finite Element Method for a Nonlinear Hyperbolic Conservation Law. Technical Report 1985-25, Mathematics Department, Charmers University of Technology, Göteborg, 1985. 
JOHSON, C.; SZEPESSY, A. The Convergence of Streamline Diffusion Finite Methods for Hyperbolic Conservation Laws. Numerical Methods for Compressible Flows - Finite Difference, Element and Volume Techniques, AMD v. 78, ASME, New York, pp. 75-91, 1986.

LAPIDUS, A. A Detached Shock Calculation by Second Order Finite Differences. J. Comp. Phys., v. 2, pp. 154-177, 1967.

MALISKA, C. R. Transferência de Calor e Mecânica dos Fluidos Computacional. ed. LTC-Livros Técnicos e Científicos Editora S.A., Rio de Janeiro RJ, 1995.

MIKHLIN, S. G. Variational Methods in Mathematical Physics. Pergamon, Oxford, 1964.

NÄVER, U. A Finite Element Method for Convection-Diffusion Problems. Ph.D. Thesis. Department of Computer Science, Charmers University of Technology, Göteborg, Sweden, 1982.

NEUMANN, J.; RICHTMEYER, R. D. A Method for Numerical Calculation of Hydrodynamic shocks. J. Appl. Phys., v. 21, pp. 232-236, 1950.

NIGRO, N.; STORTI, M.; IDELSHON, S. Two-Phase Flow Modeling in Gas-Stirred Liquid Vessels with SUPG-Stabilized Equal-Order Interpolations. International Journal for Numerical Methods in Fluids, v. 19, pp. 1-22, 1994.

NIGRO, N.; STORTI, M.; IDELSHON, S. GMRES physics preconditioner for all Reynolds and Mach numbers:numerical examples. International Journal for Numerical Methods in Fluids, v. 25, pp. 1347-1371, 1998. 
OKUSANYA, T.; DARMOFAL, D. L.; PERAIRE, J. Algebraic Multigrid for stabilized finite element discretizations of the Navier-Stokes equations. Computer Methods in Applied Mechanics and Engineering, v. 193, pp. 3667-3686, 2004.

PERAIRE, J.; VAHDATI, M.; MORGAN, K.; ZIENKIEWICS, O. C. Adaptive Remeshing for Compressible Flow Computations. Journal of Computational Physics, 72, pp. 449-466, 1987.

RAYMOND, W. H.; GARDER, A. Selective Damping in a Galerking Method for Solving Wave Problems with Variable Grids. Mon. Wheather Ver., 104, pp. 15831590, 1976.

SABUNDJIAN, G.; CABRAL, E. L. L. Aplicação do Método da Expansão em Funções Hierárquicas na Solução das Equações de Navier-Stokes para Fluidos Incompressíveis. Dissertação de Tese de Doutorado, Universidade São Paulo, São Paulo, Brasil, 1999.

SAMPAIO, P. A. B.; MOREIRA, M. L. Simulação de Escoamentos Viscosos Compressíveis e Quase-Incompressíveis. Computer Methods in Applied Mechanics and Engineering, v. 106, pp. 143-178, 1993.

SIMÕES-MOREIRA, J.R. Teoria do Escoamento Compressível. Apostila da disciplina Escoamento Compressível, Departamento de Engenharia Mecânica, Escola Politécnica da Universidade de São Paulo, $1^{\text {o }}$ período de 2002.

STORTI, M.; NIGRO, N.; IDELSHON, S. Equal-Order Interpolations: a Unified Approach to Stabilize the Incompressible and Advective Affects. Computer Methods in Applied Mechanics and Engineering, v. 143, pp. 317-331, 1997. 
TEJADA-MARTINEZ, A. E.; JANSEN, K. E. On the interaction between dynamic model dissipation and numerical dissipation due to streamline upwind/PetrovGalerkin stabilization. Computer Methods in Applied Mechanics and Engineering, v. 194, pp. 1225-1248, 2005.

TEZDUYAR, T. E.; GLOWINSKI, R.; LIOU, J. Petrov-Galerkin Methods on Multiply Connected Domains for the Vorticity-Stream Function Formulation of the Incompressible Navier-Stokes Equations. International Journal for Numerical Methods in Fluids, v. 8, pp. 1269-1290, 1988.

THOMPSON, P. A. Compressible Fluid - Dynamics. ed. McGraw-Hill, USA, 1972.

XIKUI, L.; WENHUA, W.; ZIENKIEWICZ, O. C. Implicit characteristic Galerkin method for convection-diffusion equations. International Journal for Numerical Methods in Engineering, v.47, pp. 1689-1708, 2000.

WAHLBIN, L. B. A Dissipative Galerkin Methods for the Numerical Solution of First Order Hyperbolic Equations. Mathematical Aspect of Finite Elements in Partial Differential Equations, Academic Press, New York, pp. 147-169, 1974.

WILLIS, D. J.; WHITE, J. K.; PERAIRE, J.; A pFFT accelerated linear strength BEM potential solver. Nanotechnology Conference and Trade Show, v. 2, pp. 446-449, 2004.

WONG, J. S.; DARMOFAL, D. L.; PERAIRE, J. The Solution of the compressible Euler equations at low Mach numbers using a stabilized finite element algorithm. Computer Methods in Applied Mechanics and Engineering, v. 190, pp. 5719-5737, 2001.

WOODWARD, P.; COLELLA, P. The Numerical Simulation of Two Dimensional Fluid Flow with Strong Shocks. Journal of Computational Physics, v. 54, pp. 115-173, 1984. 
ZALESAK, S. T. Fully Multi-Dimensional Flux-Corrected Transport Algorithm for Fluids. Journal of Computational Physics, v. 31, pp. 335-362, 1979.

ZIENKIEWICZ, O. C.; CHRISTIE, I.; GRIFFITHS, D. F.; MITCHELL, A. R. Finite Element Methods for Second Order Differential Equations with Significant First Derivatives. International Journal for Numerical Methods in Engineering, v. 10, pp. 1389-1396, 1976.

ZIENKIEWICZ, O. C.; LOHNER, R.; MORGAN, K.; PERAIRE, J. High Speed Compressible Flow and other Advection Dominated Problems of Fluid Mechanics. Finite Elements in Fluids, v.6, Wiley, New York, 1985.

ZIENKIEWICZ, O. C.; MORGAN, K. Finite Elements and Approximation. University of Wales, Swansea, United Kingdom. New York, 1983.

ZIENKIEWICZ, O. C.; MORGAN, K.; PERAIRE, J.; ZHU, J. Z. Some Expanding Horizons for Computational Mechanics, Error Estimates, Mesh Generation and Hyperbolic Problems. Computational Mechanics - Advances and Trends, Special Publication AMD-v.75, ASME, New York, 1986.

ZIENKIEWICZ, O. C.; ZHU, J.Z. A Simple Error Estimator and Adaptive Procedure for Practical Engineering Analysis. International Journal for Numerical Methods in Engineering, v. 24, pp. 337-357, 1987. 